\title{
Index to
}

A

Abad C, Santamaria P. Tuberculosis aneurysm of the aortic arch. 2003;126:1229 (Letter)

Abe K (see Takahashi et al). 2003;126:1461-6

Abe T (see Watanabe et al). 2003;126:1212-4 (Brief comm.)

Abe T (see Yamada et al). 2003;126:597-8 (Brief comm.)

Abraham SJK (see Osawa et al). 2003;126:604-5 (Brief comm.)

Absi TS, Sundt TM III, Tung WS, Moon M, Lee JK, Damiano RR

$\mathrm{Jr}$, Thompson RW. Altered patterns of gene expression distinguishing ascending aortic aneurysms from abdominal aortic aneurysms: complementary DNA expression profiling in the molecular characterization of aortic disease. 2003;126:344-57

Acar C. Discussion of Maisano et al. 2003;126:1987-97

Acker MA (see Oz et al). 2003;126:983-91

Acker MA (see Pilla et al). 2003;126:1467-76

Acker MA. Discussion of Pilla et al. 2003;126:1467-76

Ad N, Tian YY, Verbalis J, Imahara SD, Cox JL. The effect of the maze procedure on the secretion of arginine-vasopressin and aldosterone. 2003;126:1095-100

Adachi H (see Tanaka et al). 2003;126:837-41

Adams DH. Discussion of Blackstone et al. 2003;126:783-96

Adams DH. Discussion of Maisano et al. 2003;126:1987-97

Adatia I, Humpl T, Silkoff PE. Exhaled nitric oxide and cardiac surgery with extracorporeal circulation. 2003;126:1674-5 (Letter reply)

Adelstein DJ (see DeCamp et al). 2003;126:17-27

Adelstein DJ (see Rice et al). 2003;126:1590-6

Afanasieva OI (see Pokrovsky et al). 2003;126:1071-5

Agarwal JP (see Song et al). 2003;126:896-7 (Brief comm.)

Agnastopolous P (see Pigula et al). 2003;126:1650-2 (Brief comm.)

Agnoletti G (see Boudjemline et al). 2003;126:490-7

Agostinelli A (see Colli et al). 2003;126:881-3 (Brief comm.)

Agricola E (see Alfieri et al). 2003;126:75-9

Ahmed I (see Lim et al). 2003;126:1013-7

Aiello VD (see Binotto et al). 2003;126:1026-32

Airaksinen J (see Kakko et al). 2003;126:106-13

Akalin H (see Sirlak et al). 2003;126:666-70

Akasaka J (see Nitta et al). 2003;126:1186-8 (Brief comm.)

Akashi H (see Teshima et al). 2003;126:401-7

Akay H (see Şahin et al). 2003;126:769-73

*July, 1-310; August 311-616; September, 617-928; October, 929-1234; November, 1235-1680; December, 1681-2202.
Akcali Y (see Bilgin et al). 2003;126:1580-3

Akchurin RS (see Pokrovsky et al). 2003;126:1071-5

Akhurst T (see Flores et al). 2003;126:11-6

Akins C. Discussion of Bando et al. 2003;126:358-64

Akiyama Y (see Aramaki et al). 2003;126:853-4 (Brief comm.)

Akowauh E (see Lim et al). 2003;126:1013-7

Albanese SB (see Carotti et al). 2003;126:1839-50

Albes JM, Stöhr IM, Kaluza M, Siegemund A, Schmidt D, Vollandt R, Wahlers T. Physiological coagulation can be maintained in extracorporeal circulation by means of shed blood separation and coating. 2003;126:1504-12

Alderman EL (see Wolf et al). 2003;126:168-78

Aledort LM. Activated prothrombin complex concentrates and recombinant factor VIIa in the bleeding patient: are they appropriate and safe? 2003;126:2112-3 (Letter)

Alessandrini F (see Gaudino et al). 2003;126:1107-12

Alexandrou A (see Murthy et al). 2003;126:1162-7

Alexiou C (see Monro et al). 2003;126:511-20

Alfieri O, De Bonis M, Lapenna E, Agricola E, Quarti A, Maisano F. The "clover technique" as a novel approach for correction of post-traumatic tricuspid regurgitation. 2003;126:75-9

Alfieri O (see Maisano et al). 2003;126:1987-97

Alfieri O (see Melo et al). 2003;126:914-6 (Brief comm.)

Allen BS, Castellá M, Buckberg GD, Tan Z. Conditioned blood reperfusion markedly enhances neurologic recovery after prolonged cerebral ischemia. 2003;126:1851-8

Allen BS. Fetal cardiac surgery: simplicity versus success in a new frontier. 2003;126:1254-6 (Editorial)

Allen K. Discussion of Schmitz et al. 2003;126:1829-38

Allen SJ (see Fischer et al). 2003;126:1483-8

Almassi GH. Discussion of Wolf et al. 2003;126:168-78

Altmayer M (see Go et al). 2003;126:867-9 (Brief comm.)

Altorki NK. David Bernt Skinner (1935-2003): a thoracic surgeon and something more. 2003;126:1245-6 (In Memoriam)

Altorki NK (see Keresztes et al). 2003;126:1603-8

Amarelli C (see De Santo et al). 2003;126:826-31

Amemiya A (see Hirata et al). 2003;126:1080-6

Anagnostopoulos CE (see Toumpoulis et al). 2003;126:1672 (Letter reply)

Anderson RH (see Lunkenheimer and Anderson). 2003;126:2109-10 (Letter)

Anderson RH (see Lunkenheimer et al). 2003;126:592-6 (Brief comm.)

Andou A (see Date et al). 2003;126:476-81

Andrási T (see Szabó et al). 2003;126:651-8 
Andreotti F (see Gaudino et al). 2003;126:1107-12

Andropoulos DB, Stayer SA, McKenzie ED, Fraser CD Jr. Regional low-flow perfusion provides comparable blood flow and oxygenation to both cerebral hemispheres during neonatal aortic arch reconstruction. 2003;126:1712-7

Anegon I (see Mathieu et al). 2003;126:1449-54

Angelini GD (see Caputo et al). 2003;126:39-47

Ansley DM, Xia Z, Dhaliwal BS. The relationship between plasma free $15-\mathrm{F}_{2 \mathrm{t}}$-isoprostane concentration and early postoperative cardiac depression following warm heart surgery. 2003;126: 1222-3 (Brief comm.)

Ansley DM (see Xia et al). 2003;126:1404-10

Anyanwu AC. Authors should list confounding factors and alternative explanations for adverse events seen with new technologies. 2003;126:1663-4 (Letter)

Aoe M (see Date et al). 2003;126:476-81

Aoyagi S (see Teshima et al). 2003;126:401-7

Arai S (see Sano et al). 2003;126:504-10

Aral A (see Sirlak et al). 2003;126:666-70

Aramaki O, Shirasugi N, Akiyama Y, Ikeda Y, Yabuki S, Shimazu M, Kitajima M, Niimi M. Prolonged survival of fully allogeneic cardiac grafts in naive mice and those with sensitization induced by antigen delivery through the respiratory tract. 2003; 126:853-4 (Brief comm.)

Aramayo A (see Assad et al). 2003;126:300-2 (Brief comm.)

Aranki SF (see Greelish et al). 2003;126:365-73

Araujo E (see Ruel et al). 2003;126:1521-30

Ardehali A, Laks H, Russell H, Levine M, Shpiner R, Lackey S, Ross D. Modified reperfusion and ischemia-reperfusion injury in human lung transplantation. 2003;126:1929-34

Ardehali A (see Fischbein et al). 2003;126:216-23

Ardehali A (see Marelli et al). 2003;126:821-5

Argenziano M (see Morgan et al). 2003;126:582-3 (Brief comm.) Aribas OK (see Reisli et al). 2003;126:584-5 (Brief comm.)

Aris A. Controlled exsanguination during sternal reentry. 2003; 126:918-9 (Brief comm.)

Armour JA (see Waldmann et al). 2003;126:1792-7

Armstrong RC (see Yarbrough et al). 2003;126:1892-9

Armstrong S (see David et al). 2003;126:2118-9 (Letter reply)

Armstrong S (see Feindel et al). 2003;126:777-82

Aroca A (see Oliver et al). 2003;126:711-7

Arom KV. Discussion of Ikonomidis et al. 2003;126:2022-31

Arslanian A (see Dato et al). 2003;126:408-14

Artes M (see Hernández et al). 2003;126:2081-2 (Brief comm.)

Asai T (see Sano et al). 2003;126:504-10

Asakura T (see Gojo et al). 2003;126:885-6 (Brief comm.)

Asamura H (see Nakagawa et al). 2003;126:1134-40

Asano G (see Ishii et al). 2003;126:254-62

Asano H (see Imanaka et al). 2003;126:598-600 (Brief comm.)

Asano H (see Nishimura et al). 2003;126:1190-2 (Brief comm.)

Asano H (see Senzaki et al). 2003;126:1645-7 (Brief comm.)

Asaoka N (see Hirata et al). 2003;126:1080-6

Ashraf O (see Schmitz et al). 2003;126:645-50

Ashton RC Jr, Connery CP, Swistel DG, DeRose JJ Jr. Robotassisted lobectomy. 2003;126:292-3 (Brief comm.)

Assad RS, Zielinsky P, Kalil R, Lima G, Aramayo A, Santos A, Costa R, Marcial MB, Oliveria SA. New lead for in utero pacing for fetal congenital heart block. 2003;126:300-2 (Brief comm.)

Assad RS. Discussion of Carotti et al. 2003;126:1839-50

Assad RS. Discussion of Corno et al. 2003;126:1775-80

Assouad J (see Barthes et al). 2003;126:1199-200 (Brief comm.)

Athanassiadi K (see Gerazounis et al). 2003;126:774-6

Attar NA, Sablayrolles J-L, Nataf P. Giant atherosclerotic aneurysm of the left anterior descending artery. 2003;126:888-90 (Brief comm.)

Austin EH III (see Yeh et al). 2003;126:589-91 (Brief comm.)

Auvinen MI (see Sihvo et al). 2003;126:1952-7

Avunduk MC (see Reisli et al). 2003;126:584-5 (Brief comm.)

Aybek T (see Wimmer-Greinecker et al). 2003;126:465-8 (Evolving tech.)

Azizzadeh A (see Estrera et al). 2003;126:1288-95

\section{B}

Baan J (see Leeuwenburgh et al). 2003;126:2105-6 (Letter reply) Bacha EA, Cao Q-L, Starr JP, Waight D, Ebeid MR, Hijazi ZM. Perventricular device closure of muscular ventricular septal defects on the beating heart: technique and results. 2003;126: 1718-23

Bacha EA (see Jonas et al). 2003;126:1765-74

Baciewicz FA Jr. Bivalirudin as alternative to both danaparoid and heparin in off-pump coronary artery bypass grafting. 2003;126: 2108-9 (Letter)

Backer CL. Coarctation: the search for the Holy Grail. 2003;126: 329-31 (Editorial)

Backer CL. Discussion of McMahon et al. 2003;126:1781-7

Badack S (see Klima et al). 2003;126:1568-74 (Evolving tech.)

Badia A (see Barthes et al). 2003;126:1199-200 (Brief comm.)

Badiwala MV (see Verma et al). 2003;126:1886-91

Baenziger O (see Gessler et al). 2003;126:718-25

Bährle S (see Szabó et al). 2003;126:651-8

Bailey M (see Damiano et al). 2003;126:2016-21

Bailey M (see Moazami et al). 2003;126:2097-8 (Brief comm.)

Bains MS (see Park et al). 2003;126:726-31

Baker CJ (see Wells et al). 2003;126:498-503

Balc1 AE (see Eren et al). 2003;126:574-81

Balc1 AE (see Eren et al). 2003;126:855-7 (Brief comm.)

Balsam LB (see Kofidis et al). 2003;126:2113-4 (Letter)

Banbury MK, White JA, Blackstone EH, Cosgrove DM III. Vacuum-assisted venous return reduces blood usage. 2003;126: 680-7

Bandali KS, Belanger MP, Wittnich C. Does hyperoxia affect glucose regulation and transport in the newborn? 2003;126: 1730-5

Bando K, Kobayashi J, Hirata M, Satoh T, Niwaya K, Tagusari O, Nakatani S, Yagihara T, Kitamura S. Early and late stroke after mitral valve replacement with a mechanical prosthesis: risk factor analysis of a 24-year experience. 2003;126:358-64

Banfi C (see Rescigno et al). 2003;126:1196-7 (Brief comm.)

Barber D (see Dowling et al). 2003;126:1271-8

Bargenda S (see Klima et al). 2003;126:1568-74 (Evolving tech.)

Baron F (see Hvass et al). 2003;126:818-20

Barr ML (see Wells et al). 2003;126:498-503

Barron M (see Bolooki et al). 2003;126:374-85 
Bart RD (see DiPerna et al). 2003;126:1129-33

Bartelings MM (see Lalezari et al). 2003;126:1053-60

Bartels C (see Boyce et al). 2003;126:420-7

Barthes FLP, D'Attellis N, Assouad J, Badia A, Souilamas R, Riquet M. Chylous leak after cervical mediastinoscopy. 2003; 126:1199-200 (Brief comm.)

Bartlett J (see Jonas et al). 2003;126:1765-74

Baskett R. Discussion of Bolooki et al. 2003;126:374-85

Baskett RJF, Nanton MA, Warren AE, Ross DB. Human leukocyte antigen-DR and $\mathrm{ABO}$ mismatch are associated with accelerated homograft valve failure in children: implications for therapeutic interventions. 2003;126:232-9

Baslaim G, Hussain A, Kouatli A, Jamjoom A. Bovine valved xenograft conduits in the extracardiac Fontan procedure. 2003; 126:586-8 (Brief comm.)

Basoglu A, Celik B, Sengul AT. Giant parenchymal bronchogenic cyst mimicking hydropneumothorax. 2003;126:1201-2 (Brief comm.)

Baste J-M (see Litzler et al). 2003;126:1204-7 (Brief comm.)

Batista RV (see Lunkenheimer et al). 2003;126:592-6 (Brief comm.)

Battaglia F (see Bonacchi et al). 2003;126:1302-13

Bauer U (see Kaemmerer et al). 2003;126:1048-52

Baufreton C, de Brux JL. Coagulation, fibrinolysis, and cell activation in patients and in shed mediastinal blood during coronary artery bypass grafting with a new heparin-coated surface. 2003;126:2116 (Letter)

Baumann FG (see Bizekis et al). 2003;126:659-665. Correction 2003; $126: 1680$

Baumgartner RW (see Georgiadis et al). 2003;126:1638-9 (Brief comm.)

Baumgartner WA. Discussion of Jonas et al. 2003;126:1765-74

Beard M (see Goldberg et al). 2003;126:1168-73

Beck JM (see Jonjev et al). 2003;126:1880-5

Beghi C (see Colli et al). 2003;126:881-3 (Brief comm.)

Belanger MP (see Bandali et al). 2003;126:1730-5

Beldholm BR, Wilson MK, Gallagher RM, Caminer D, King MJ, Glanville A. Reconstruction of the trachea with a tubed radial forearm free flap. 2003;126:545-50

Bellinger DC, Wypij D, duPlessis AJ, Rappaport LA, Jonas RA, Wernovsky G, Newburger JW. Neurodevelopmental status of eight years in children with dextro-transposition of the great arteries: the Boston Circulatory Arrest Trial. 2003;126:1385-96

Bellinger DC (see Jonas et al). 2003;126:1765-74

Bellinger DC (see Wypij et al). 2003;126:1397-403

Bellomi M (see Pastorino et al). 2003;126:1906-10

Benfield JR (see Roberts et al). 2003;126:1597-602

Benito F (see Oliver et al). 2003;126:711-7

Bennink GBWE (see Walhout et al). 2003;126:521-8

Benussi S (see Melo et al). 2003;126:914-6 (Brief comm.)

Berg A (see Trachiotis et al). 2003;126:2065-71

Bernbaum J (see Gaynor et al). 2003;126:1736-45

Bertel O (see Genoni et al). 2003;126:288-9 (Brief comm.)

Bessou J-P (see Litzler et al). 2003;126:1204-7 (Brief comm.)

Bevandic SM (see Ghanayem et al). 2003;126:1367-77

Bianchi C (see Ruel et al). 2003;126:1521-30

Bilgin M, Akcali Y, Oguzkaya F. Extrapleural regional versus systemic analgesia for relieving postthoracotomy pain: a clin- ical study of bupivacaine compared with metamizol. 2003;126: 1580-3

Binotto MA, Higuchi MdL, Aiello VD. Left ventricular remodeling in hearts with tricuspid atresia: morphologic observations and possible basis for ventricular dysfunction after surgery. 2003;126:1026-32

Biondi A (see Onorati et al). 2003;126:1345-51

Birkmeyer NJ (see Blackstone et al). 2003;126:783-96

Bisleri G, Morgan JA, Deng MC, Mancini DM, Oz MC. Should HIV-positive recipients undergo heart transplantation? 2003; 126:1639-40 (Brief comm.)

Bisleri G (see Morgan et al). 2003;126:1624-33

Bizekis C, Pintucci G, Derivaux CC, Saponara F, Kim J-H, Hyman KM, Sharony R, Grossi EA, Baumann FG, Mignatti P, Galloway AC. Activation of mitogen-activated protein kinases during preparation of vein grafts and modulation by a synthetic inhibitor. 2003;126:659-665. Correction 2003;126:1680

Blaauwgeers JLG (see van der Maten et al). 2003;126:740-3

Black E (see Mussa et al). 2003;126:1798-805

Blackstone EH, Cosgrove DM, Jamieson WRE, Birkmeyer NJ, Lemmer JH Jr, Miller DC, Butchart EG, Rizzoli G, Yacoub M, Chai A. Prosthesis size and long-term survival after aortic valve replacement. 2003;126:783-96

Blackstone EH (see Banbury et al). 2003;126:680-7

Blackstone EH (see DeCamp et al). 2003;126:17-27

Blackstone EH (see Gillinov et al). 2003;126:313-6 (Editorial)

Blackstone EH (see Koch et al). 2003;126:2032-43

Blackstone EH (see Koch et al). 2003;126:2044-51

Blackstone EH (see Rice et al). 2003;126:1590-6

Blasio A (see Maisano et al). 2003;126:1987-97

Bloch W (see Tossios et al). 2003;126:1513-20

Block MI. Negative aspects of preoperative delay in early stage non-small cell lung cancer. 2003;126:610 (Letter reply)

Blom AS (see Pilla et al). 2003;126:1467-76

Bodian C (see Hagl et al). 2003;126:1005-12

Bohn D (see van Dongen et al). 2003;126:703-10

Boice T (see Dowling et al). 2003;126:1271-8

Boineau JP (see Damiano et al). 2003;126:2016-21

Boineau JP (see Prasad et al). 2003;126:1822-8

Bolli R (see Boyce et al). 2003;126:420-7

Bolooki H, DeMarchena E, Mallon SM, Katariya K, Barron M, Bolooki M, Thurer RJ, Novak S, Duncan RC. Factors affecting late survival rate after surgical remodeling of left ventricular aneurysms. 2003;126:374-85

Bolooki M (see Bolooki et al). 2003;126:374-85

Bonacchi M, Prifti E, Battaglia F, Frati G, Sani G, Popoff G. In situ retrocaval skeletonized right internal thoracic artery anastomosed to the circumflex system via transverse sinus: technical aspects and postoperative outcome. 2003;126:1302-13

Bonanomi G, Rebello K, Lebouitz K, Riviere C, Di Martino E, Vorp D, Zenati MA. Microelectromechanical systems for endoscopic cardiac surgery. 2003;126:851-2 (Evolving tech.)

Bonanomi G, Schwarzman D, Francischelli D, Hebsgaard K, Zenati MA. A new device for beating heart bipolar radiofrequency atrial ablation. 2003;126:1859-66

Bonato R (see Bottio et al). 2003;126:1194-5 (Brief comm.)

Bonatti J (see Hangler et al). 2003;126:391-400

Bonavida B (see Fischbein et al). 2003;126:216-23 
Bonnet D (see Boudjemline et al). 2003;126:490-7

Bonnet D (see Corno et al). 2003;126:1775-80

Bonser M (see Harrington et al). 2003;126:638-44

Bonser RS (see Harrington et al). 2003;126:638-44

Boonstra PW (see Drenth et al). 2003;126:1184-5 (Brief comm.)

Border WL, Syed AU, Michelfelder EC, Khoury P, Uzark KC, Manning PB, Pearl JM. Impaired systemic ventricular relaxation affects postoperative short-term outcome in Fontan patients. 2003;126:1760-4

Borger MA (see Doll et al). 2003;126:1662 (Letter reply)

Borger MA (see Doll et al). 2003;126:1663 (Letter reply)

Borger MA (see Doll et al). 2003;126:2120 (Letter reply)

Borger MA (see Peterson et al). 2003;126:1314-9

Borst C, Eikelaar JHR, Meijer R, van Boven WJ, Klein P, Gründeman PF. Future technologic innovations for intraoperative visualization of native coronary artery and graft anastomoses. 2003;126:305 (Letter reply)

Borst C (see Scheltes et al). 2003;126:191-9 (Evolving tech.)

Bortolotti U (see Bottio et al). 2003;126:99-105

Bortolotti U (see De Carlo et al). 2003;126:337-43

Bortolotti U (see Mecozzi et al). 2003;126:1198-9 (Brief comm.) Bostwick TK (see Klena et al). 2003;126:857-9 (Brief comm.)

Botsios S (see Cebi et al). 2003;126:2074-7 (Brief comm.)

Bottio T, Pittarello G, Bonato R, Fagiolo U, Gerosa G. Lifethreatening anaphylactic shock caused by porcine heparin intravenous infusion during mitral valve repair. 2003;126:1194-5 (Brief comm.)

Bottio T, Rizzoli G, Vida V, Casarotto D, Gerosa G. Double crisscross sternal wiring and chest wound infections: a prospective randomized study. 2003;126:1352-6

Bottio T, Thiene G, Pettenazzo E, Ius P, Bortolotti U, Rizzoli G, Valfré C, Casarotto D, Valente M. Hancock II bioprosthesis: a glance at the microscope in mid-long-term explants. 2003;126: 99-105

Bottio T (see Rizzoli et al). 2003;126:66-74

Bouchart F (see Litzler et al). 2003;126:1204-7 (Brief comm.)

Boudjemline Y, Bonnet D, Massih TA, Agnoletti G, Iserin F, Jaubert F, Sidi D, Vouhé P. Use of bovine jugular vein to reconstruct the right ventricular outflow tract: early results. 2003;126:490-7

Boval B (see Houël). 2003;126:1636-7 (Brief comm.)

Bove EL, de Leval MR, Migliavacca F, Guadagni G, Dubini G. Computational fluid dynamics in the evaluation of hemodynamic performance of cavopulmonary connections after the Norwood procedure for hypoplastic left heart syndrome. 2003; 126:1040-7

Boyce SW, Bartels C, Bolli R, Chaitman B, Chen JC, Chi E, Jessel A, Kereiakes D, Knight J, Thulin L, Theroux P, GUARDIAN Study Investigators. Impact of sodium-hydrogen exchange inhibition by cariporide on death or myocardial infarction in high-risk $\mathrm{CABG}$ surgery patients: results of the CABG surgery cohort of the GUARDIAN study. 2003;126:420-7

Boyd WD, Stahl KD. The Janus syndrome: a perspective on a new era of computer-enhanced robotic cardiac surgery. 2003;126: 625-30 (Editorial)

Bradley SM, Simsic JM, Mulvihill DM. Hypoventilation improves oxygenation after bidirectional superior cavopulmonary connection. 2003;126:1033-9
Bradley SM (see Ikonomidis et al). 2003;126:2022-31

Bradley SM (see Simsic et al). 2003;126:186-90

Braun J, Voigt PG, Versteegh MIM, Dion RAE. Restrictive mitral annuloplasty in refractory cardiogenic shock with acute postinfarction mitral insufficiency and intact papillary muscle. 2003; 126:284-6 (Brief comm.)

Bremner RM (see DiPerna et al). 2003;126:1129-33

Bremner RM. Discussion of Okumura et al. 2003;126:1922-8

Brenner W. Discussion of Roberts et al. 2003;126:1597-602

Bresson J (see Marelli et al). 2003;126:821-5

Bresson S (see Marelli et al). 2003;126:821-5

Brimioulle S (see Wauthy et al). 2003;126:1434-41

Brister SJ (see Ibrahim et al). 2003;126:959-64

Brister SJ (see Lawton et al). 2003;126:936-8 (Editorial)

Brockman DJ (see Pilla et al). 2003;126:1467-76

Brodherr-Heberlein S (see Kaemmerer et al). 2003;126:1048-52

Brouse CF (see Greilich et al). 2003;126:1498-503

Brown DW, Gauvreau K, Moran AM, Jenkins KJ, Perry SB, del Nido PJ, Colan SD. Clinical outcomes and utility of cardiac catheterization prior to superior cavopulmonary anastomosis. 2003;126:272-81

Brown K (see McMahon et al). 2003;126:1781-7

Browne SM, Halligan PW, Wade DT, Taggart DP. Postoperative hypoxia is a contributory factor to cognitive impairment after cardiac surgery. 2003;126:1061-4

Brugière O (see Duariat et al). 2003;126:875-7 (Brief comm.)

Brugière O (see Thabut et al). 2003;126:469-75

Buchanan MR (see Ibrahim et al). 2003;126:959-64

Buckberg GD.The helix and the heart. 2003;126:921-2 (Letter reply)

Buckberg GD (see Allen et al). 2003;126:1851-8

Buckberg GD (see Castellá et al). 2003;126:1442-8

Buckberg GD. Discussion of Bolooki et al. 2003;126:374-85

Buckley C (see Delius et al). 2003;126:2095-6 (Brief comm.)

Budde JM (see Corvera et al). 2003;126:1549-54

Budillon AM (see Colli et al). 2003;126:881-3 (Brief comm.)

Buehler T (see Frese et al). 2003;126:748-54

Buhmann V (see Szabó et al). 2003;126:651-8

Burch M (see McMahon et al). 2003;126:1781-7

Burdick J (see Friedberg et al). 2003;126:1963-7 (Evolving tech.)

Burzotta F (see Gaudino et al). 2003;126:1107-12

Butany J (see Fedak et al). 2003;126:797-806

Butchart EG (see Blackstone et al). 2003;126:783-96

Buxton BF (see Shah et al). 2003;126:1320-7

Byhahn C (see Wimmer-Greinecker et al). 2003;126:465-8 (Evolving tech.)

Byrne JG (see Greelish et al). 2003;126:365-73

\section{C}

Calafiore AM (see Gaudino et al). 2003;126:1076-9

Caldarola A (see Maisano et al). 2003;126:1987-97

Calvi S (see Melo et al). 2003;126:914-6 (Brief comm.)

Cambillau M (see Zegdi et al). 2003;126:1867-74

Cameron DE. Discussion of Corno et al. 2003;126:1775-80

Camillo CJ (see Prasad et al). 2003;126:1822-8

Caminer D (see Beldholm et al). 2003;126:545-50

Camuset J (see Duariat et al). 2003;126:875-7 (Brief comm.) 
Cangır AK (see Şahin et al). 2003;126:769-73

Canosa C (see Gaudino et al). 2003;126:1076-9

Cao Q-L (see Bacha et al). 2003;126:1718-23

Cao-Danh H (see Friehs et al). 2003;126:263-71

Caputo M, Reeves B, Marchetto G, Mahesh B, Lim K, Angelini GD. Radial versus right internal thoracic artery as a second arterial conduit for coronary surgery: early and midterm outcomes. 2003;126:39-47

Cardella JA (see Fischer et al). 2003;126:1174-80

Carotti A, Emma F, Picca S, Iannace E, Albanese SB, Grigioni M, Meo F, Sciarra M, Di Donato RM. Inflammatory response to cardiac bypass in ewe fetuses: effects of steroid administration or continuous hemodiafiltration. 2003;126:1839-50

Carotti A, Marino B, Di Donato RM. Influence of chromosome 22q11.2 microdeletion on surgical outcome after treatment of tetralogy of Fallot with pulmonary atresia. 2003;126:1666-7 (Letter)

Carpentier A (see Zegdi et al). 2003;126:1867-74

Carr SR (see Friedberg et al). 2003;126:1963-7 (Evolving tech.)

Carrel T (see Gessler et al). 2003;126:718-25

Carrella G (see Sortini et al). 2003;126:608-9 (Letter)

Carrella G (see Sortini et al). 2003;126:609-10 (Letter)

Carrier M. Bivalirudin as alternative to both danaparoid and heparin in off-pump coronary artery bypass grafting. 2003;126: 2109 (Letter reply)

Carson S (see Mickleborough et al). 2003;126:950-8

Casarotto D (see Bottio et al). 2003;126:99-105

Casarotto D (see Bottio et al). 2003;126:1352-6

Casarotto D (see Rizzoli et al). 2003;126:66-74

Casher J (see Williams et al). 2003;126:436-41

Caskey MP (see Wolf et al). 2003;126:168-78

Casson AG. Discussion of Lerut et al. 2003;126:1121-8

Casta A (see Pigula et al). 2003;126:1650-2 (Brief comm.)

Castellá M, Buckberg GD, Saleh S, Tan Z, Ignarro LJ. A new role for cardioplegic buffering: should acidosis or calcium accumulation be counteracted to salvage jeopardized hearts? 2003;126: 1442-8

Castellá M (see Allen et al). 2003;126:1851-8

Castier Y (see Duariat et al). 2003;126:875-7 (Brief comm.)

Castier Y (see Thabut et al). 2003;126:469-75

Cava JR (see Ghanayem et al). 2003;126:1367-77

Cebi N, Johannes F, Botsios S, Walterbusch G. Intraparenchymal replacement of the left pulmonary artery with implantation of segmental arteries in a 26-year-old patient. 2003;126:2074-7 (Brief comm.)

Cejna M (see Czerny et al). 2003;126:916-8 (Brief comm.)

Celik B (see Basoglu et al). 2003;126:1201-2 (Brief comm.)

Cerasulo F (see Onorati et al). 2003;126:1345-51

Cerfolio RJ. Discussion of Reed et al. 2003;126:1943-51

Cesario A (see Margaritora et al). 2003;126:1664-5 (Letter)

Chachques J. Discussion of Saito et al. 2003;126:114-23

Chachques JC. Discussion of Pilla et al. 2003;126:1467-76

Chai A (see Blackstone et al). 2003;126:783-96

Chaitman B (see Boyce et al). 2003;126:420-7

Chaitman BR (see Gavard et al). 2003;126:807-13

Chan D (see Weinstein et al). 2003;126:529-36

Chan RK (see Shah et al). 2003;126:1320-7
Chaney MA (see Schwann and Chaney). 2003;126:1261-4 (Editorial)

Chang R-K (see Gupta et al). 2003;126:1746-52

Chang Y-L (see Huang et al). 2003;126:2090-2 (Brief comm.)

Chang Y-L (see Lee et al). 2003;126:298-9 (Brief comm.)

Chang Y-S (see Hwang et al). 2003;126:1643-4 (Brief comm.)

Channon KM (see Mussa et al). 2003;126:1798-805

Cheema FH (see Kherani et al). 2003;126:1634-5 (Brief comm.)

Cheema FH (see Morgan et al). 2003;126:582-3 (Brief comm.)

Cheema FH (see Morgan et al). 2003;126:1188-90 (Brief comm.)

Cheema FH (see Morgan et al). 2003;126:1624-33

Chen C-W (see Huang et al). 2003;126:2090-2 (Brief comm.)

Chen C-W (see Lee et al). 2003;126:298-9 (Brief comm.)

Chen JC (see Boyce et al). 2003;126:420-7

Chen J-S (see Huang et al). 2003;126:2090-2 (Brief comm.)

Chen RP-C (see Waldmann et al). 2003;126:1792-7

Chen Y-S (see Yu et al). 2003;126:80-9

Chester MR (see Conant et al). 2003;126:448-54

Chew D (see Koster et al). 2003;126:906-7 (Brief comm.)

Chi E (see Boyce et al). 2003;126:420-7

Chi E (see Gavard et al). 2003;126:807-13

Chi L (see Greilich et al). 2003;126:1498-503

Chiappini B, Martìn-Suàrez S, LoForte A, Di Bartolomeo R, Marinelli G. Surgery for atrial fibrillation using radiofrequency catheter ablation. 2003;126:1788-91

Chidel MA (see DeCamp et al). 2003;126:17-27

Chidel MA (see Rice et al). 2003;126:1590-6

Chien N-C (see Huang et al). 2003;126:2090-2 (Brief comm.)

Chien N-C (see Lee et al). 2003;126:298-9 (Brief comm.)

Chitwood WR. Discussion of Maisano et al. 2003;126:1987-97

Chiu K-M (see Lin et al). 2003;126:2011-5

Chiu RC-J. Myogenesis after myocardial stem cell transplantation. 2003;126:2117 (Letter reply)

Chiu RC-J (see Saito et al). 2003;126:114-23

Chiyo M, Sekine Y, Iwata T, Tatsumi K, Yasufuku K, Iyoda A, Otsuji M, Yoshida S, Shibuya K, Iizasa T, Saitoh Y, Fujisawa T. Impact of interstitial lung disease on surgical morbidity and mortality for lung cancer: analyses of short-term and long-term outcomes. 2003;126:1141-6

Chu D, Sullivan CC, Weitzman MD, Du L, Wolf PL, Jamieson SW, Thistlethwaite PA. Direct comparison of efficiency and stability of gene transfer into the mammalian heart using adeno-associated virus versus adenovirus vectors. 2003; 126:671-9

Chu J-J (see Hwang et al). 2003;126:1643-4 (Brief comm.)

Chu K-M (see Murthy et al). 2003;126:1162-7

Chu S-H (see Lin et al). 2003;126:2011-5

Chu S-H (see Yu et al). 2003;126:80-9

Clancy RR (see Gaynor et al). 2003;126:1736-45

Clark S (see Perreas et al). 2003;126:606-7 (Brief comm.)

Clark SC, Rao JN, Flecknell PA, Dark JH. Pentoxifylline is as effective as leukocyte depletion for modulating pulmonary reperfusion injury. 2003;126:2052-7

Clark SC (see Zacharias et al). 2003;126:1210-1 (Brief comm.)

Clarke DR. Discussion of Baskett et al. 2003;126:232-9

Clarke J (see Jahangiri et al). 2003;126:894-895 (Brief comm.) Correction 2003;126:1680

Clavier E (see Litzler et al). 2003;126:1204-7 (Brief comm.) 
Cohen G (see McMahon et al). 2003;126:1781-7

Cohen M (see Madan et al). 2003;126:1657-9 (Brief comm.)

Cohn LH (see Farivar and Cohn). 2003;126:969-76

Cohn LH (see Greelish et al). 2003;126:365-73

Coia L (see Goldberg et al). 2003;126:1168-73

Colan SD (see Brown et al). 2003;126:272-81

Colarusso P (see Goldberg et al). 2003;126:1168-73

Coleman RE (see Gonzalez-Stawinski et al). 2003;126:1900-5

Colletti NJ (see Morgan et al). 2003;126:1624-33

Colli A, Budillon AM, DeCicco G, Agostinelli A, Nicolini F,

Tzialtas D, Zoffoli G, Corradi D, Maestri R, Beghi C, Gherli T.

Recurrence of a right ventricular hemangioma. 2003;126:881-3 (Brief comm.)

Colvin SB (see Grossi et al). 2003;126:922 (Letter)

Conant AR, Shackcloth MJ, Oo AY, Chester MR, Simpson AWM, Dihmis WC. Phenoxybenzamine treatment is insufficient to prevent spasm in the radial artery: the effect of other vasodilators. 2003;126:448-54

Conlan AA, Sandor A. Total thoracoscopic pneumonectomy: indications and technical considerations. 2003;126:2083-5 (Brief comm.)

Conlan AA (see McNamee et al). 2003;126:861-3 (Brief comm.)

Conlan AA (see McNamee et al). 2003;126:910-2 (Brief comm.)

Connery CP (see Ashton et al). 2003;126:292-3 (Brief comm.)

Cooley DA. A milestone in cardiovascular surgery. 2003;126: 1243-4 (Hon. Guest's address)

Cooley DA. Discussion of Blackstone et al. 2003;126:783-96

Cooper J. Discussion of Force and Patterson. 2003;126:1247-50 (Clinical path. conf.)

Cooper WA (see Corvera et al). 2003;126:1549-54

Coosemans W (see Lerut et al). 2003;126:1121-8

Cordoba M (see Hernández et al). 2003;126:2081-2 (Brief comm.)

Corno AF, Bonnet D, Sekarski N, Sidi D, Vouhé P, von Segesser LK. Remote control of pulmonary blood flow: initial clinical experience. 2003;126:1775-80

Corno AF (see Marino and Corno). 2003;126:1225-6 (Letter)

Corradi D (see Colli et al). 2003;126:881-3 (Brief comm.)

Corvera JS, Morris CD, Budde JM, Velez DA, Puskas JD, Lattouf OM, Cooper WA, Guyton RA, Vinten-Johansen J. Pretreatment with phenoxybenzamine attenuates the radial artery's vasoconstrictor response to $\alpha$-adrenergic stimuli. $2003 ; 126$ : 1549-54

Cosgrove DM III (see Banbury et al). 2003;126:680-7

Cosgrove DM (see Blackstone et al). 2003;126:783-96

Cosgrove DM (see Svensson et al). 2003;126:892-3 (Brief comm.)

Cosola T (see Morgan et al). 2003;126:1624-33

Costa R (see Assad et al). 2003;126:300-2 (Brief comm.)

Cote RJ. Occult metastases: real harm or false alarm? 2003;126: 332-3 (Editorial)

Cotrufo M (see De Santo et al). 2003;126:826-31

Cotrufo M (see Onorati et al). 2003;126:1345-51

Couper GS (see Greelish et al). 2003;126:365-73

Cowan DB (see Friehs et al). 2003;126:263-71

Cox CS Jr (see Fischer et al). 2003;126:1483-8

Cox J. Discussion of Raman et al. 2003;126:1357-66

Cox JL. Atrial fibrillation I: a new classification system. 2003;126: 1686-92 (Editorial)
Cox JL. Atrial fibrillation II: rationale for surgical treatment. 2003;126:1693-9 (Editorial)

Cox JL (see Ad et al). 2003;126:1095-100

Cox JL (see Damiano et al). 2003;126:2016-21

Cox JL (see Prasad et al). 2003;126:1822-8

Crabbe DL (see He et al). 2003;126:56-65

Crawford FA Jr. Thoracic surgery education: responding to a changing environment. 2003;126:1235-42 (Pres. address)

Crawford FA Jr (see Ikonomidis et al). 2003;126:2022-31

Crawford FA Jr (see Yarbrough et al). 2003;126:1892-9

Criner GJ (see Petersen et al). 2003;126:2086-90 (Brief comm.)

Croce S (see Filosso et al). 2003;126:1215-7 (Brief comm.)

Crumbley AJ III (see Ikonomidis et al). 2003;126:2022-31

Cryer CW (see Lunkenheimer et al). 2003;126:592-6 (Brief comm.)

Crystal RG (see Mahtabifard et al). 2003;126:28-38

Crystal RG (see Merritt et al). 2003;126:1609-17

Culver JP (see Friedberg et al). 2003;126:1963-7 (Evolving tech.)

Cusimano RJ (see Verma et al). 2003;126:1555-60 (Evolving tech.)

Czerny M, Fleck T, Zimpfer D, Dworschak M, Hofmann W, Hutschala D, Dunkler D, Ehrlich M, Wolner E, Grabenwoger M. Risk factors of mortality and permanent neurologic injury in patients undergoing ascending aortic and arch repair. 2003;126: 1296-301

Czerny M, Fleck T, Zimpfer D, Kilo J, Sandner D, Cejna M, Lammer J, Wolner E. Grabenwoger M. Combined repair of an aortic arch aneurysm by sequential transposition of the supraaortic branches and endovascular stent-graft placement. 2003; 126:916-8 (Brief comm.)

Daddi G (see Puma et al). 2003;126:1998-2002

Daddi N (see Suda et al). 2003;126:1155-61

Daddi N (see Tagawa et al). 2003;126:1147-54

Dagenais F (see Mathieu et al). 2003;126:1449-54

D’Agostino JA (see Gaynor et al). 2003;126:1736-45

Dahinden C (see Gessler et al). 2003;126:718-25

Daitoku K (see Takahashi et al). 2003;126:600-2 (Brief comm.)

Damiano R. Discussion of Raman et al. 2003;126:1357-66

Damiano RJ Jr, Gaynor SL, Bailey M, Prasad S, Cox JL, Boineau JP, Schuessler RP. The long-term outcome of patients with coronary disease and atrial fibrillation undergoing the Cox maze procedure. 2003;126:2016-21

Damiano RJ Jr (see Dickey et al). 2003;126:1259-60 (Editorial)

Damiano RJ Jr (see Moazami et al). 2003;126:2097-8 (Brief comm.)

Damiano RJ Jr (see Olsen et al). 2003;126:992-9

Damiano RJ Jr (see Prasad et al). 2003;126:1822-8

Damiano RR Jr (see Absi et al). 2003;126:344-57

D'Amico TA (see Gonzalez-Stawinski et al). 2003;126:1900-5

D'Amico TA. Discussion of DeCamp et al. 2003;126:17-27

Danchin N (see Gavard et al). 2003;126:807-13

Danek SJ (see Klena et al). 2003;126:857-9 (Brief comm.)

Daniel MA (see Wolf et al). 2003;126:168-78

Danzmayr M (see Hangler et al). 2003;126:391-400

Dark JH (see Clark et al). 2003;126:2052-7 
Dark JH (see Zacharias et al). 2003;126:1210-1 (Brief comm.)

Dasika UK, Kanter KR, Vincent R. Nickel allergy to the percutaneous patent foramen ovale occluder and subsequent systemic nickel allergy. 2003;126:2112 (Letter)

Date H, Aoe M, Nagahiro I, Sano Y, Andou A, Matsubara H, Goto K, Tedoriya T, Shimizu N. Living-donor lobar lung transplantation for various lung diseases. 2003;126:476-81

Dato GMA, Arslanian A, Di Marzio P, Filosso PL, Ruffini E. Posttraumatic and iatrogenic foreign bodies in the heart: report of fourteen cases and review of the literature. 2003;126:408-14

D'Attellis N (see Barthes et al). 2003;126:1199-200 (Brief comm.)

David TE, Ivanov J, Armstrong S, Rakowski H. Asymptomatic mitral valve incompetence: is there evidence for surgery? 2003; 126:2118-9 (Letter reply)

David TE, Ivanov J. Is degenerative calcification of the native aortic valve similar to calcification of bioprosthetic heart valves? 2003;126:939-41 (Editorial)

David TE (see Fedak et al). 2003;126:797-806

David TE (see Feindel et al). 2003;126:777-82

Davierwala PM, Maganti M, Yau TM. Decreasing significance of left ventricular dysfunction and reoperative surgery in predicting coronary artery bypass grafting-associated mortality: a twelve-year study. 2003;126:1335-44

Davies A (see Taggart et al). 2003;126:1087-94

Davis JT (see Weinstein et al). 2003;126:529-36

De Bonis M (see Alfieri et al). 2003;126:75-9

De Bonis M (see Maisano et al). 2003;126:1987-97

de Brux JL (see Baufreton and de Brux). 2003;126:2116 (Letter)

De Carlo M, Milano AD, Nardi C, Mecozzi G, Bortolotti U. Serial Doppler echocardiographic evaluation of small-sized Sorin Bicarbon prostheses. 2003;126:337-43

De Feo M (see Onorati et al). 2003;126:1345-51

de la Morena M (see Lee et al). 2003;126:295-7 (Brief comm.)

de Leval MR (see Bove et al). 2003;126:1040-7

de Leval MR (see McMahon et al). 2003;126:1781-7

De Leyn P (see Lerut et al). 2003;126:1121-8

de Mol BA (see van den Goor et al). 2003;126:1101-6

de Perrot M (see Fischer et al). 2003;126:1174-80

de Sa MPL (see Fedak et al). 2003;126:797-806

De Santo LS, Romano G, Amarelli C, Onorati F, Torella M, Renzulli A, Galdieri N, Cotrufo M. Surgical repair of acute type A aortic dissection: continuous pulmonary perfusion during retrograde cerebral perfusion prevents lung injury in a pilot study. 2003;126:826-31

Dearani JA. Discussion of Damiano et al. 2003;126:2016-21

DeCamp MM, Rice TW, Adelstein DJ, Chidel MA, Rybicki LA, Murthy SC, Blackstone EH. Value of accelerated multimodality therapy in stage IIIA and IIIB non-small cell lung cancer. 2003;126:17-27

DeCamp MM (see Rice et al). 2003;126:1590-6

DeCicco G (see Colli et al). 2003;126:881-3 (Brief comm.)

Decker G (see Lerut et al). 2003;126:1121-8

del Nido PJ (see Brown et al). 2003;126:272-81

del Nido PJ (see Friehs et al). 2003;126:263-71

del Nido PJ (see Jonas et al). 2003;126:1765-74

del Nido PJ (see Marshall et al). 2003;126:1427-33
Delius RE, Buckley C, Walters HL III. Cervical cannulation for resternotomy in pediatric patients. 2003;126:2095-6 (Brief comm.)

DeMarchena E (see Bolooki et al). 2003;126:374-85

DeMeester SR. Lymph node involvement in esophageal adenocarcinoma: if you see one, have you seen them all? 2003;126: 947-9 (Editorial)

Demers P, Moffatt S, Oyer PE, Hunt SA, Reitz BA, Robbins RC. Long-term results of heart transplantation in patients older than 60 years. 2003; 126:224-31

Demeyere R (see Sergeant et al). 2003;126:1279-87

Demirsoy E (see Sonmez et al). 2003;126:1662-3 (Letter)

Deneke T (see Laczkovics et al). 2003;126:2119-20 (Letter)

Deng MC (see Bisleri et al). 2003;126:1639-40 (Brief comm.)

Derilus FE (see Scarborough et al). 2003;126:1561-7 (Evolving tech.)

Derivauz CC (see Bizekis et al). 2003;126:659-665. Correction 2003; $126: 1680$

DeRose JJ Jr (see Ashton et al). 2003;126:292-3 (Brief comm.)

DeRuiter MC (see Grauss et al). 2003;126:2003-10

Detterbeck FC. Discussion of Ferguson. 2003;126:1935-42

Detterbeck FC. Discussion of Reed et al. 2003;126:1943-51

Devagourou V (see Hussain et al). 2003;126:879-80 (Brief comm.)

Dhaliwal BS (see Ansley et al). 2003;126:1222-3 (Brief comm.)

Di Bartolomeo R (see Chiappini et al). 2003;126:1788-91

Di Bartolomeo R (see Pacini et al). 2003;126:900-2 (Brief comm.)

Di Castelnuovo A (see Gaudino et al). 2003;126:1107-12

Di Donato R (see Formigari et al). 2003;126:1753-9

Di Donato RM (see Carotti et al). 2003;126:1839-50

Di Donato RM (see Carotti et al). 2003;126:1666-7 (Letter)

Di Martino E (see Bonanomi et al). 2003;126:851-2 (Evolving tech.)

Di Marzio P (see Dato et al). 2003;126:408-14

Di Mauro M (see Gaudino et al). 2003;126:1076-9

Di Russo GB (see Hawkins et al). 2003;126:247-53

Di Simone M (see Pacini et al). 2003;126:900-2 (Brief comm.)

DiBardino DJ, Espada R, Seu P, Goss JA. Management of complicated pneumatocele. 2003;126:859-61 (Brief comm.)

Dickey J, Damiano RJ Jr, Ungerleider R. Our surgical culture of blame: a time for change. 2003;126:1259-60 (Editorial)

Dienemann H (see Pfannschmidt et al). 2003;126:732-9

Dieter RA Jr. Foreign bodies expectorated through the pneumonectomy stump. 2003;126:2104 (Letter)

Dietl CA, Kasirajan K, Pett SB, Wernly JA. Off-pump management of aortic arch aneurysm by using an endovascular thoracic stent graft. 2003;126:1181-3 (Brief comm.)

Dihmis WC (see Conant et al). 2003;126:448-54

DiMaio JM (see Greilich et al). 2003;126:1498-503

Dinarello CA (see Selzman et al). 2003;126:688-93

Dine A (see Dowling et al). 2003;126:1271-8

Dion RAE (see Braun et al). 2003;126:284-6 (Brief comm.)

Dion RAE (see Schoof et al). 2003;126:282-3 (Brief comm.)

Dion RAE. Discussion of Maisano et al. 2003;126:1987-97

DiPerna CA, Bart RD, Sievers EM, Ma Y, Starnes VA, Bremner RM. Cyclooxygenase-2 inhibition decreases primary and metastatic tumor burden in a murine model of orthotopic lung adenocarcinoma. 2003;126:1129-33

Dipp MA (see Mussa et al). 2003;126:1798-805 
Djapardy V (see Taggart et al). 2003;126:1087-94

Dodos F (see Tossios et al). 2003;126:1513-20

Doenst T (see Fischer-Rasokat and Doenst). 2003;126:1806-12

Dogan S (see Wimmer-Greinecker et al). 2003;126:465-8 (Evolving tech.)

Doi K (see Iwakura et al). 2003;126:1113-20

Doll N, Borger MA, Mohr FW. Atrioesophageal fistula: Is it an unavoidable complication of radiofrequency ablation? 2003; 126:1663 (Letter reply)

Doll N, Borger MA, Mohr FW. Esophageal perforation during left atrial radiofrequency ablation: is the risk too high? 2003;126: 1662 (Letter reply)

Doll N, Mohr FW, Borger MA. Esophageal perforation during left atrial radiofrequency ablation. 2003;126:2120 (Letter reply)

Domkowski PW, Smith ML, Gonyon DL Jr, Drye C, Wooten MK, Levin LS, Wolfe WG. Evaluation of vacuum-assisted closure in the treatment of poststernotomy mediastinitis. 2003;126: 386-90

Donahue DM (see Gaissert et al). 2003;126:744-7

Donati G (see Filosso et al). 2003;126:1215-7 (Brief comm.)

Donati MB (see Gaudino et al). 2003;126:1107-12

Doss M, Woehleke T, Wood JP, Martens S, Greinecker GW, Moritz A. The clamshell approach for the treatment of extensive thoracic aortic disease. 2003;126:814-7

Doty D. Discussion of Lai et al. 2003;126:1978-86

Douvrin F (see Litzler et al). 2003;126:1204-7 (Brief comm.)

Dowdy KB (see Yarbrough et al). 2003;126:1489-97

Dowdy KB (see Yarbrough et al). 2003;126:1892-9

Dowling R, Thielmeier K, Ghaly A, Barber D, Boice T, Dine A. Improved pain control after cardiac surgery: results of a randomized, double-blind, clinical trial. 2003;126:1271-8

Downey RJ (see Park et al). 2003;126:726-31

Downey RJ (see Reed et al). 2003;126:1943-51

Drenth DJ, Verhoeven ELG, Prins TR, Waterbolk TW, Boonstra PW. Relocation of supra-aortic vessels to facilitate endovascular treatment of a ruptured aortic arch aneurysm. 2003;126: 1184-5 (Brief comm.)

Drinkwater DC. Discussion of Wells et al. 2003;126:498-503

Drossos GE (see Toumpoulis et al). 2003;126:1672 (Letter reply)

Drouet L (see Houël). 2003;126:1636-7 (Brief comm.)

Drye C (see Domkowski et al). 2003;126:386-90

Du L (see Chu et al). 2003;126:671-9

Duariat G, Brugière $\mathrm{O}$, Mal H, Camuset J, Castier Y, Lesèche G, Fournier M. Refractory chylothorax after lung transplantation for lymphangioleiomyomatosis successfully cured with instillation of povidone. 2003;126:875-7 (Brief comm.)

Dubini G (see Bove et al). 2003;126:1040-7

Dullum M (see Lawton et al). 2003;126:936-8 (Editorial)

Dullum MK (see Wolf et al). 2003;126:168-78

Duncan BW, Mee RBB, Prieto LR, Rosenthal GL, Mesia CI, Qureshi A, Tucker OP, Rhodes JF, Latson LA. Staged repair of tetralogy of Fallot with pulmonary atresia and major aortopulmonary collateral arteries. 2003;126:694-702

Duncan BW (see Lupinetti et al). 2003;126:240-6

Duncan RC (see Bolooki et al). 2003;126:374-85

Dunkler D (see Czerny et al). 2003;126:1296-301

Dunning J (see Stoica et al). 2003;126:2072-4 (Brief comm.)

duPlessis AJ (see Bellinger et al). 2003;126:1385-96
duPlessis AJ (see Jonas et al). 2003;126:1765-74

duPlessis AJ (see Wypij et al). 2003;126:1397-403

Durdu MS (see Sirlak et al). 2003;126:666-70

Dworschak M (see Czerny et al). 2003;126:1296-301

Dyamenahalli U (see Lupinetti et al). 2003;126:240-6

Dziadziuszko R (see Rzyman et al). 2003;126:755-60

\section{E}

Ebeid MR (see Bacha et al). 2003;126:1718-23

Ectors N (see Lerut et al). 2003;126:1121-8

Edmonds HL Jr (see Yeh et al). 2003;126:589-91 (Brief comm.)

Edwards NM (see Morgan et al). 2003;126:1624-33

Ehrlich M (see Czerny et al). 2003;126:1296-301

Eijsman L (see van den Goor et al). 2003;126:1101-6

Eikelaar JHR (see Borst et al). 2003;126:305 (Letter reply)

El Oakley RM, Ooi OC, Lee CN. Is there an evidence in favor of off-pump coronary artery bypass? 2003;126:1668 (Letter)

Elliott M (see McMahon et al). 2003;126:1781-7

Ellis J (see Saxena et al). 2003;126:90-8

Elsebaey A (see Hvass et al). 2003;126:818-20

Emma F (see Carotti et al). 2003;126:1839-50

Enomoto K (see Nakagawa et al). 2003;126:563-7

Enön S (see Şahin et al). 2003;126:769-73

Entwistle JC III (see Samuels et al). 2003;126:2100-1 (Brief comm.)

Entwistle JWC (see Rangappa et al). 2003;126:124-32

Eren Ş, Eren MN, Balcı AE. Pneumonectomy in children for destroyed lung and the long-term consequences. 2003;126: 574-81

Eren Ş (see Eren et al). 2003;126:855-7 (Brief comm.)

Eren MN, Balcı AE, Eren Ş. Benign intrapulmonary teratoma: report of a case. 2003;126:855-7 (Brief comm.)

Eren MN (see Eren et al). 2003;126:574-81

Eren NT (see Sirlak et al). 2003;126:666-70

Ergin MA (see Hagl et al). 2003;126:1005-12

Erhardt G (see Schoenburg et al). 2003;126:1455-60

Erhardt L (see Gavard et al). 2003;126:807-13

Eryilmaz S (see Sirlak et al). 2003;126:666-70

Escobar GP (see Yarbrough et al). 2003;126:1489-97

Escobar GP (see Yarbrough et al). 2003;126:1892-9

Esmailian F (see Marelli et al). 2003;126:821-5

Esmailzadeh B (see Schmitz et al). 2003;126:645-50

Espada R (see DiBardino et al). 2003;126:859-61 (Brief comm.)

Estrera AL, Miller CC III, Huynh TTT, Azizzadeh A, Porat EE, Vinnerkvist A, Ignacio C, Sheinbaum R, Safi HJ. Preoperative and operative predictors of delayed neurologic deficit following repair of thoracoabdominal aortic aneurysm. 2003;126:1288-95

Estrera AL. Discussion of Absi et al. 2003;126:344-57

Eyileten A (see Sirlak et al). 2003;126:666-70

Ezhov MV (see Pokrovsky et al). 2003;126:1071-5

\section{$\mathrm{F}$}

Fabiani J-N (see Zegdi et al). 2003;126:1867-74

Fabre O (see Zegdi et al). 2003;126:1867-74

Fagiolo U (see Bottio et al). 2003;126:1194-5 (Brief comm.) 
Fal JM (see Kherani et al). 2003;126:1634-5 (Brief comm.)

Falk V, Walther T, Stein H, Jacobs S, Walther C, Rastan A, Wimmer-Greinecker G, Mohr FW. Facilitated endoscopic beating heart coronary artery bypass grafting using a magnetic coupling device. 2003;126:1575-9 (Evolving tech.)

Falk V (see Klima et al). 2003;126:1568-74 (Evolving tech.)

Falk V (see Wolf and Falk). 2003;126:634-7 (Editorial)

Farivar AS (see Naidu et al). 2003;126:200-7

Farivar AS (see Naidu et al). 2003;126:482-9

Farivar RS, Cohn LH. Hypercholesterolemia is a risk factor for bioprosthetic valve calcification and explanation. 2003;126: 969-76

Farma J (see Goldberg et al). 2003;126:1168-73

Farrell DM (see Jonas et al). 2003;126:1765-74

Fazel S, Weisel RD, Li R-K. A few critical aspects—and Achilles heels-of tissue engineering approaches to restore injured myocardium. 2003;126:2114-6 (Letter reply)

Fazio F (see Pastorino et al). 2003;126:1906-10

Fedak PWM, de Sa MPL, Verma S, Nili N, Kazemian P, Butany J, Strauss BH, Weisel RD, David TE. Vascular matrix remodeling in patients with bicuspid aortic valve malformations: implications for aortic dilatation. 2003;126:797-806

Fedak PWM (see Ohno et al). 2003;126:1537-48

Fedak PWM (see Verma et al). 2003;126:1555-60 (Evolving tech.)

Fedak PWM (see Verma et al). 2003;126:1886-91

Fedeli C (see Puma et al). 2003;126:1998-2002

Feindel CM, Tufail Z, David TE, Ivanov J, Armstrong S. Mitral valve surgery in patients with extensive calcification of the mitral annulus. 2003;126:777-82

Feindel CM (see Peterson et al). 2003;126:1314-9

Ferguson MK. Optimal management when unsuspected N2 nodal disease is identified during thoracotomy for lung cancer: costeffectiveness analysis. 2003;126:1935-42

Fernando HC. Discussion of Gonzalez-Stawinski et al. 2003;126: 1900-5

Ferrari VA (see Pilla et al). 2003;126:1467-76

Ferrazzi P (see Rescigno et al). 2003;126:1196-7 (Brief comm.)

Ferri L, Fraser R, Gaboury L, Mulder D. Epstein-Barr virusassociated pulmonary leiomyosarcoma arising twenty-nine years after renal transplantation. 2003;126:877-9 (Brief comm.)

Fieuws S (see Lerut et al). 2003;126:1121-8

Filho JDF (see Lunkenheimer et al). 2003;126:592-6 (Brief comm.)

Filosso PL, Donati G, Ruffini E, Croce S, Papotti M, Oliaro A, Mancuso M. Primary malignant melanoma of the bronchus intermedius. 2003;126:1215-7 (Brief comm.)

Filosso PL. Possible Tx N2 M0 atypical bronchial carcinoid associated with Cushing syndrome. 2003;126:1224-5 (Letter)

Filosso PL (see Dato et al). 2003;126:408-14

Fimmers R (see Schmitz et al). 2003;126:645-50

Fischbein MP, Yun J, Laks H, Irie Y, Oslund-Pinderski L, Fishbein MC, Bonavida B, Ardehali A. Regulated interleukin-10 expression prevents chronic rejection of transplanted hearts. 2003; $126: 216-23$

Fischer S, de Perrot M, Liu M, MacLean AA, Cardella JA, Imai Y, Suga M, Keshavjee S. Interleukin-10 gene transfection of donor lungs ameliorates posttransplant cell death by a switch from cellular necrosis to apoptosis. 2003;126:1174-80
Fischer S, Gohrbandt G, Meyer A, Simon AR, Haverish A, Strüber M. Should lungs from donors with severe acute pulmonary embolism be accepted for transplantation? The Hanover experience. 2003;126:1641-3 (Brief comm.)

Fischer UM, Cox CS Jr, Allen SJ, Stewart RH, Mehlhorn U, Laine GA. The antioxidant $N$-acetylcysteine preserves myocardial function and diminishes oxidative stress after cardioplegic arrest. 2003;126:1483-8

Fischer-Rasokat U, Doenst T. Insulin-induced improvement of postischemic recovery is abolished by inhibition of protein kinase $\mathrm{C}$ in rat heart. 2003;126:1806-12

Fishbein MC (see Fischbein et al). 2003;126:216-23

Fleck T (see Czerny et al). 2003;126:916-8 (Brief comm.)

Fleck T (see Czerny et al). 2003;126:1296-301

Flecknell PA (see Clark et al). 2003;126:2052-7

Flora R (see Gaudino et al). 2003;126:1968-71

Florek JC (see Lunkenheimer et al). 2003;126:592-6 (Brief comm.)

Flores R (see Park et al). 2003;126:726-31

Flores RM, Akhurst T, Gonen M, Larson SM, Rusch VW. Positron emission tomography defines metastatic disease but not locoregional disease in patients with malignant pleural mesothelioma. 2003; $126: 11-6$

Floten HS. Discussion of Lai et al. 2003;126:1978-86

Follette DM (see Roberts et al). 2003;126:1597-602

Follis F. Discussion of Sergeant et al. 2003;126:1279-87

Fonsi GB (see Puma et al). 2003;126:1998-2002

Forbess JM. Pre-stage II mortality after the Norwood operation: addressing the next challenge. 2003;126:1257-8 (Editorial)

Forbess JM (see Jonas et al). 2003;126:1765-74

Force S, Patterson GA. Clinical-pathologic conference in general thoracic surgery: pulmonary blastoma. 2003;126:1247-50

Force SD, Lau CL, Moazami N, Trulock EP, Patterson GA. Bilateral lung transplantation and pulmonary artery reconstruction in a patient with chronic obstructive pulmonary disease, and a giant pulmonary artery aneurysm. 2003;126:864-6 (Brief comm.)

Formigari R, Toscano A, Giardini A, Gargiulo G, Di Donato R, Picchio FM, Pasquini L. Prevalence and predictors of neoaortic regurgitation after arterial switch operation for transposition of the great arteries. 2003;126:1753-9

Fornès P (see Zegdi et al). 2003;126:1867-74

Fournier M (see Duariat et al). 2003;126:875-7 (Brief comm.)

Fournier M (see Thabut et al). 2003;126:469-75

Foweraker J (see Lim et al). 2003;126:1013-7

Fox J (see Greelish et al). 2003;126:365-73

Fraga C (see Naidu et al). 2003;126:482-9

Francischelli D (see Bonanomi et al). 2003;126:1859-66

Fraser CD Jr (see Andropoulos et al). 2003;126:1712-7

Fraser CD Jr. Discussion of Ghanayem et al. 2003;126:1367-77

Fraser CD Jr. Discussion of Jonas et al. 2003;126:1765-74

Fraser R (see Ferri et al). 2003;126:877-9 (Brief comm.)

Fraser VJ (see Olsen et al). 2003;126:992-9

Frati G (see Bonacchi et al). 2003;126:1302-13

Fratz S, Geiger R, Kresse H, Roemer G, Hennig M, Sebening W, Hess J. Pulmonary blood pressure, not flow, is associated with net endothelin-1 production in the lungs of patients with con- 
genital heart disease and normal pulmonary vascular resistance. 2003;126:1724-9

Fratz S (see Kaemmerer et al). 2003;126:1048-52

Fremes SE (see Mallidi and Fremes). 2003;126:2111-2 (Letter reply)

Frenkel G (see Medalion et al). 2003;126:1875-9

Frese S, Schaper M, Kuster J-R, Miescher D, Jäättelä M, Buehler $\mathrm{T}$, Schmid RA. Cell death induced by down-regulation of heat shock protein 70 in lung cancer cell lines is p53-independent and does not require DNA cleavage. 2003;126:748-54

Fried PW (see Wechsler and Fried). 2003;126:1681-2 (Editorial)

Friedberg JS, Skema C, Burdick J, Yodh AG, Carr SR, Culver JP. A novel technique for light delivery through branched or bent anatomic structures. 2003;126:1963-7 (Evolving tech.)

Friehs I, Cao-Danh H, Stamm C, Cowan DB, McGowan FX, del Nido PJ. Postnatal increase in insulin-sensitive glucose transporter expression is associated with improved recovery of postischemic myocardial function. 2003;126:263-71

Frisbee SJ (see Ghanayem et al). 2003;126:1367-77

Frommelt PC (see Ghanayem et al). 2003;126:1367-77

Frucht H (see Goldberg et al). 2003;126:1168-73

Fujii T (see Ohno et al). 2003;126:1537-48

Fujii Y (see Okumura et al). 2003;126:1922-8

Fujisawa T (see Chiyo et al). 2003;126:1141-6

Fujita M (see Iwakura et al). 2003;126:1113-20

Fujiwara K (see Yamagishi et al). 2003;126:1218-9 (Brief comm.)

Fukada J (see Yamada et al). 2003;126:597-8 (Brief comm.)

Fukuda I, Imazuru T. Intentional delay of surgery for acute type A dissection with stroke. 2003;126:290-1 (Brief comm.)

Fukuda I (see Takahashi et al). 2003;126:600-2 (Brief comm.)

Fukuda T, Kashima I, Yoshiba S. Surgically created double orifice repair of tricuspid regurgitation in infants with congenital heart disease. 2003;126:1220-1 (Brief comm.)

Fukuhara K (see Okumura et al). 2003;126:1922-8

Fukui K (see Takahashi et al). 2003;126:600-2 (Brief comm.)

Fukui S (see Yoshitatsu et al). 2003;126:1531-6

Fukumoto Y (see Mori et al). 2003;126:415-9

Fukunaga S (see Teshima et al). 2003;126:401-7

Fukuyama N (see Hayashi et al). 2003;126:1813-21

Fukuyama S (see Yamaguchi et al). 2003;126:870-2 (Brief comm.)

Fuller J (see Shah et al). 2003;126:1972-7

Fuller TC (see Hawkins et al). 2003;126:247-53

\section{G}

Gaboury L (see Ferri et al). 2003;126:877-9 (Brief comm.)

Gaissert H (see Grikscheit et al). 2003;126:537-44

Gaissert HA, Grillo HC, Wright CD, Donahue DM, Wain JC, Mathisen DJ. Complication of benign tracheobronchial strictures by self-expanding metal stents. 2003;126:744-7

Galai N, Israeli A, Zitser-Gurevich Y, Simchen E. Is discharge policy a balanced decision between clinical considerations and hospital ownership policy? The CABG example. 2003;126: 1018-25

Galajda Z, Szentkirályi I, Péterffy Á. Brachial artery cannulation. 2003;126:2107 (Letter reply)

Galdieri N (see De Santo et al). 2003;126:826-31

Galiñanes M (see Ghosh and Galiñanes). 2003;126:133-42
Galiñanes M (see Loubani et al). 2003;126:143-7

Galla JD (see Hagl et al). 2003;126:1005-12

Gallagher RM (see Beldholm et al). 2003;126:545-50

Gallo R (see Gavard et al). 2003;126:807-13

Galloway AC (see Bizekis et al). 2003;126:659-665. Correction 2003; $126: 1680$

Gandara DR (see Roberts et al). 2003;126:1597-602

Gandhi SK (see Pigula et al). 2003;126:1650-2 (Brief comm.)

Gao Y-J, Yang H, Teoh K, Lee RMKW. Detrimental effects of papaverine on the human internal thoracic artery. 2003;126: 179-85

Gargiulo G (see Formigari et al). 2003;126:1753-9

Garrido A (see Oliver et al). 2003;126:711-7

Garrido MJ (see Kherani et al). 2003;126:1634-5 (Brief comm.)

Gaudino M, Di Castelnuovo A, Zamparelli R, Andreotti F, Burzotta F, Iacoviello L, Glieca F, Alessandrini F, Nasso G, Donati MB, Maseri A, Schiavello R, Possati G. Genetic control of postoperative systemic inflammatory reaction and pulmonary and renal complications after coronary artery surgery. 2003; 126:1107-12

Gaudino M, Di Mauro M, Iacò AL, Canosa C, Vitolla G, Calafiore AM Immediate flow reserve of $\mathrm{Y}$ thoracic artery grafts: an intraoperative flowmetric study. 2003;126:1076-9

Gaudino M, Tondi P, Serricchio M, Spatuzza P, Santoliquido A, Flora R, Girola F, Nasso G, Pola P, Possati G. Atherosclerotic involvement of the radial artery in patients with coronary artery disease and its relation with midterm radial artery graft patency and endothelial function. 2003;126:1968-71

Gauvreau K (see Brown et al). 2003;126:272-81

Gavard JA, Chaitman BR, Sakai S, Stocke K, Danchin N, Erhardt L, Gallo R, Chi E, Jessel A, Théroux P, GUARDIAN Investigators. Prognostic significance of elevated creatine kinase $M B$ after coronary bypass surgery and after an acute coronary syndrome: results from the GUARDIAN trial. 2003; $126: 807-13$

Gaynor JW, Gerdes M, Zackai EH, Bernbaum J, Wernovsky G, Clancy RR, Newman MF, Saunders AM, Heagerty PJ, D'Agostino JA, McDonald-McGinn D, Nicolson SC, Spray TL, Jarvik GP. Apolipoprotein E genotype and neurodevelopmental sequelae of infant cardiac surgery. 2003;126:1736-45

Gaynor JW (see Madan et al). 2003;126:1657-9 (Brief comm.)

Gaynor JW. Discussion of Ghanayem et al. 2003;126:1367-77

Gaynor SL (see Damiano et al). 2003;126:2016-21

Geha A (see Shuhaiber et al). 2003;126:1660-1 (Letter)

Geiger R (see Fratz et al). 2003;126:1724-9

Geiselseder G (see Mair et al). 2003;126:1378-84

Genoni M, Tavakoli R, Hofer C, Bertel O, Turina M. Clopidogrel before urgent coronary artery bypass graft. 2003;126:288-9 (Brief comm.)

Georgiadis D, Hempel A, Baumgartner RW, Zerkowski H-R. Doppler microembolic signals during cardiac surgery: comparison between arterial line and middle cerebral artery. 2003;126: 1638-9 (Brief comm.)

Gerazounis M, Athanassiadi K, Kalantzi N, Moustardas M. Spontaneous pneumomediastinum: a rare benign entity. 2003;126: 774-6

Gerdes M (see Gaynor et al). 2003;126:1736-45

Gerosa G (see Bottio et al). 2003;126:1194-5 (Brief comm.) 
Gerosa G (see Bottio et al). 2003;126:1352-6

Gessler P, Pfenninger J, Pfammatter J-P, Carrel T, Baenziger O, Dahinden C. Plasma levels of interleukin-8 and expression of interleukin-8 receptors on circulating neutrophils and monocytes after cardiopulmonary bypass in children. 2003;126:718-25

Ghaly A (see Dowling et al). 2003;126:1271-8

Ghanayem NS, Hoffman GM, Mussatto KA, Cava JR, Frommelt PC, Rudd NA, Steltzer MM, Bevandic SM, Frisbee SJ, Jaquis RDB, Litwin SB, Tweddell JS. Home surveillance program prevents interstage mortality after the Norwood procedure. 2003;126:1367-77

Gherli T (see Colli et al). 2003;126:881-3 (Brief comm.)

Ghosh S, Galiñanes M. Protection of the human heart with ischemic preconditioning during cardiac surgery: role of cardiopulmonary bypass. 2003;126:133-42

Ghosh S (see Loubani et al). 2003;126:143-7

Giamundo B (see Rescigno et al). 2003;126:1196-7 (Brief comm.)

Giardini A (see Formigari et al). 2003;126:1753-9

Gibson WC (see Yarbrough et al). 2003;126:1892-9

Gillinov AM. A self-retaining retractor for the maze procedure. 2003;126:287-8 (Brief comm.)

Gillinov AM, Blackstone EH, Rodriguez LL. Prosthesis-patient size: measurement and clinical implications. 2003;126:313-6 (Editorial)

Gillinov AM, McCarthy PM, Pettersson G, Lytle BW, Rice TW. Esophageal perforation during left atrial radiofrequency ablation: is the risk too high? 2003;126:1661-2 (Letter)

Ginsberg R. Discussion of DeCamp et al. 2003;126:17-27

Ginsburg ME (see Morgan et al). 2003;126:582-3 (Brief comm.)

Girola F (see Gaudino et al). 2003;126:1968-71

Gittenberger-de Groot AC (see Grauss et al). 2003;126:2003-10

Gittenberger-de Groot AC (see Lalezari et al). 2003;126:1053-60

Gitter R (see Mair et al). 2003;126:1378-84

Glansdorp AG (see van Dongen et al). 2003;126:703-10

Glanville A (see Beldholm et al). 2003;126:545-50

Glazer H. Discussion of Force and Patterson. 2003;126:1247-50 (Clinical path. conf.)

Glieca F (see Gaudino et al). 2003;126:1107-12

Go T, Altmayer M, Richter M, Macchiarini P. Decompressing manubriectomy under apneic oxygenation to release the median thoracic outlet compartment in Bechterew disease. 2003; 126:867-9 (Brief comm.)

Goddard M (see Stoica et al). 2003;126:2072-4 (Brief comm.)

Gohrbandt G (see Fischer et al). 2003;126:1641-3 (Brief comm.)

Gojo S, Kyo S, Sato H, Nishimura M, Asakura T, Ito H, Koyama K. Successful LVAS and RVAS-ECMO support in a patient with fulminant myocarditis who failed to recover from ventricular fibrillation with PCPS and IABP. 2003;126:885-6 (Brief comm.)

Goldberg M, Farma J, Lamper C, Colarusso P, Coia L, Frucht H, Goosenberg E, Beard M, Weiner LM. Survival following intensive preoperative combined modality therapy with paclitaxel, cisplatin, 5-fluorouracil, and radiation in resectable esophageal carcinomas: a phase I report. 2003;126:1168-73

Goldemund A (see Gulbins et al). 2003;126:890-1 (Brief comm.)

Goldman A (see McMahon et al). 2003;126:1781-7

Gonen M (see Flores et al). 2003;126:11-6
Gonyon DL Jr (see Domkowski et al). 2003;126:386-90

González A (see Oliver et al). 2003;126:711-7

Gonzalez-Stawinski GV, Lemaire A, Merchant F, O'Halloran E, Coleman RE, Harpole DH, D'Amico TA. A comparative analysis of positron emission tomography and mediastinoscopy in staging non-small cell lung cancer. 2003;126:1900-5

Goodfellow J (see Madhavan and Goodfellow). 2003;126:1671-2 (Letter)

Goodkin H (see Jonas et al). 2003;126:1765-74

Goosenberg E (see Goldberg et al). 2003;126:1168-73

Gordon I (see Shah et al). 2003;126:1320-7

Gordon I (see Shah et al). 2003;126:1972-7

Goss JA (see DiBardino et al). 2003;126:859-61 (Brief comm.)

Goto K (see Date et al). 2003;126:476-81

Gotoh M, Okamoto T, Yamamoto Y, Liu D, Kameyama K, Hayashi E, Huang C-L, Yokomise H. Development of a canine model of pulmonary emphysema and imaging of the emphysematous lung with infrared thoracoscopy. 2003;126:1916-21

Govindan R. Discussion of Force and Patterson. 2003;126:1247-50 (Clinical path. conf.)

Grabenwoger M (see Czerny et al). 2003;126:916-8 (Brief comm.)

Grabenwoger M (see Czerny et al). 2003;126:1296-301

Graham ANJ (see Jones et al). 2003;126:303 (Letter)

Granone P (see Margaritora et al). 2003;126:1664-5 (Letter)

Grauss RW, Hazekamp MG, van Vliet S, Gittenberger-de Groot AC, DeRuiter MC. Decellularization of rat aortic valve allografts reduces leaflet destruction and extracellular matrix remodeling. 2003;126:2003-10

Greelish JP, Cohn LH, Leacche M, Mitchell M, Karavas A, Fox J, Byrne JG, Aranki SF, Couper GS. Minimally invasive mitral valve repair suggests earlier operations for mitral valve disease. 2003; $126: 365-73$

Gregorio R (see Onorati et al). 2003;126:1345-51

Greilich PE, Brouse CF, Whitten CW, Chi L, DiMaio JM, Jessen ME. Antifibrinolytic therapy during cardiopulmonary bypass reduces proinflammatory cytokine levels: a randomized, double-blind, placebo-controlled study of $\epsilon$-aminocaproic acid and aprotinin. 2003;126:1498-503

Greinecker GW (see Doss et al). 2003;126:814-7

Griepp RB (see Hagl et al). 2003;126:1005-12

Grigioni M (see Carotti et al). 2003;126:1839-50

Grikscheit T, Ochoa ER, Srinivasan A, Gaissert H, Vacanti JP Tissue-engineered esophagus: experimental substitution by onlay patch or interposition. 2003;126:537-44

Grillo HC (see Gaissert et al). 2003;126:744-7

Grillone G (see Pacini et al). 2003;126:900-2 (Brief comm.)

Gross C (see Mair et al). 2003;126:1378-84

Grossi EA, Sharony R, Colvin SB. Mitral valve in ischemic versus idiopathic dilated cardiomyopathy. 2003;126:922 (Letter)

Grossi EA (see Bizekis et al). 2003;126:659-665. Correction 2003; 126:1680

Groundstroem K (see Kakko et al). 2003;126:106-13

Groussard O (see Thabut et al). 2003;126:469-75

Grover FL (see Selzman et al). 2003;126:688-93

Gründeman PF (see Borst et al). 2003;126:305 (Letter reply)

Grunkemeier GL, Wu YX. Study design in valve surgery and outcome. 2003;126:1661 (Letter reply) 
Grunkemeier GL (see Wu and Grunkemeier). 2003;126:1670 (Letter reply)

Gu A (see He et al). 2003;126:56-65

Gu J (see Xia et al). 2003;126:1404-10

Guadagni G (see Bove et al). 2003;126:1040-7

GUARDIAN Investigators (see Boyce et al). 2003;126:420-7

GUARDIAN Investigators (see Gavard et al). 2003;126:807-13

Gulbins H, Goldemund A, Uhlig A, Pritisanac A, Meiser B, Reichart B. Implantation of an autologously endothelialized homograft. 2003;126:890-1 (Brief comm.)

Gummert JF (see Oz et al). 2003;126:983-91

Gupta A, Odim J, Levi D, Chang R-K, Laks H. Staged repair of pulmonary atresia with ventricular septal defect and major aortopulmonary collateral arteries: experience with 104 patients. 2003;126:1746-52

Guyton RA (see Corvera et al). 2003;126:1549-54

Guzik TJ (see Mussa et al). 2003;126:1798-805

\section{$\mathrm{H}$}

Haas-Hubscher C (see Litzler et al). 2003;126:1204-7 (Brief comm.)

Habazettl H (see Koster et al). 2003;126:906-7 (Brief comm.)

Haddy S, Starnes VA. Lobar transplantation. 2003;126:2106 (Letter)

Hagl C, Galla JD, Spielvogel D, Bodian C, Lansman SL, Squitieri R, Ergin MA, Griepp RB. Diabetes and evidence of atherosclerosis are major risk factors for adverse outcome after elective thoracic aortic surgery. 2003;126:1005-12

Hagl C (see Karck et al). 2003;126:602-4 (Brief comm.)

Hagl S (see Szabó et al). 2003;126:651-8

Halligan PW (see Browne et al). 2003;126:1061-4

Halstead JC (see Lim et al). 2003;126:1013-7

Hamano K (see Sugi et al). 2003;126:568-73

Hamilton JRL (see Zacharias et al). 2003;126:1210-1 (Brief comm.)

Hammel D (see Nabavi et al). 2003;126:160-7

Hammer WJ (see McClurken et al). 2003;126:293-5 (Brief comm.)

Hangler HB, Nagele G, Danzmayr M, Mueller L, Ruttmann E, Laufer G, Bonatti J. Modification of surgical technique for ascending aortic atherosclerosis: impact on stroke reduction in coronary artery bypass grafting. 2003;126:391-400

Hankinson T (see Williams et al). 2003;126:436-41

Hanley FL. Discussion of Carotti et al. 2003;126:1839-50

Harada H (see Okada et al). 2003;126:558-62

Hardin AE (see Yarbrough et al). 2003;126:1892-9

Hare DL (see Shah et al). 2003;126:1320-7

Harjula AL (see Sihvo et al). 2003;126:1952-7

Harpole DH (see Gonzalez-Stawinski et al). 2003;126:1900-5

Harpole DH (see Reed et al). 2003;126:1943-51

Harrington DK, Bonser M, Moss, A, Heafield MTE, Riddoch MJ, Bonser RS. Neuropsychometric outcome following aortic arch surgery: a prospective randomized trial of retrograde cerebral perfusion. 2003; 126:638-44

Hartz RS, Swain JA, Mickleborough L. Sixty-year perspective on coronary artery bypass grafting in women. 2003;126:620-2 (Editorial)

Hasan A (see Zacharias et al). 2003;126:1210-1 (Brief comm.)
Hasegawa T (see Ootaki et al). 2003;126:1411-6

Hasegawa T (see Ootaki et al). 2003;126:1655-6 (Brief comm.)

Hatsuoka S (see Hirata et al). 2003;126:1080-6

Hauptman E (see Medalion et al). 2003;126:1875-9

Haverich A (see Karck et al). 2003;126:602-4 (Brief comm.)

Haverich A (see Klima et al). 2003;126:1568-74 (Evolving tech.)

Haverich A (see Leyh et al). 2003;126:1000-4

Haverish A (see Fischer et al). 2003;126:1641-3 (Brief comm.)

Hawkins J. Discussion of Baskett et al. 2003;126:232-9

Hawkins JA, Hillman ND, Lambert LM, Jones J, Di Russo GB, Profaizer T, Fuller TC, Minich LL, Williams RV, Shaddy RE. Immunogenicity of decellularized cryopreserved allografts in pediatric cardiac surgery: comparison with standard cryopreserved allografts. 2003;126:247-53

Hawkins JA. Discussion of Lupinetti et al. 2003;126:240-6

Hayashi E (see Gotoh et al). 2003;126:1916-21

Hayashi Y, Sawa Y, Fukuyama N, Miyamoto Y, Takahashi T, Nakazawa H, Matsuda H. Leukocyte-depleted terminal blood cardioplegia provides superior myocardial protective effects in association with myocardium-derived nitric oxide and peroxynitrite production for patients undergoing prolonged aortic crossclamping for more than 120 minutes. 2003;126:1813-21

Hayashida N (see Teshima et al). 2003;126:401-7

Hazekamp M (see Schoof et al). 2003;126:282-3 (Brief comm.)

Hazekamp MG (see Grauss et al). 2003;126:2003-10

Hazekamp MG (see Lalezari et al). 2003;126:1053-60

He G-W (see Zou et al). 2003;126:208-15

He G-W. Discussion of Blackstone et al. 2003;126:783-96

He H, Shirota T, Yasui H, Matsuda T. Canine endothelial progenitor cell-lined hybrid vascular graft with nonthrombogenic potential. 2003;126:455-64

He K-1, Shimizu J, Yi G-h, Gu A, Kashem MA, Crabbe DL, Popilskis S, Wu EX, Santamore WP, Melvin D, Wang J. Left ventricular systolic performance in failing heart improved acutely by left ventricular reshaping. 2003;126:56-65

Heafield MTE (see Harrington et al). 2003;126:638-44

Heagerty PJ (see Gaynor et al). 2003;126:1736-45

Hebsgaard K (see Bonanomi et al). 2003;126:1859-66

Heelan RT (see Reed et al). 2003;126:1943-51

Hein S (see Schoenburg et al). 2003;126:1455-60

Helbing WA (see Leeuwenburgh et al). 2003;126:2105-6 (Letter reply)

Hellmich M (see Tossios et al). 2003;126:1513-20

Hempel A (see Georgiadis et al). 2003;126:1638-9 (Brief comm.)

Hendrick JW (see Yarbrough et al). 2003;126:1489-97

Hendrick JW (see Yarbrough et al). 2003;126:1892-9

Hennig M (see Fratz et al). 2003;126:1724-9

Herbella FAM. Short esophagi and long career. 2003;126:1668 (Letter)

Herden T (see Leyh et al). 2003;126:1000-4

Hernández H, Varela A, Cordoba M, Madrigal L, Artes M. Videothoracoscopic extrapleural insertion of Walter Lorenz Surgical bar for pectus excavatum. 2003;126:2081-2 (Brief comm.)

Hervé P (see Zegdi et al). 2003;126:1867-74

Hess J (see Fratz et al). 2003;126:1724-9

Hess J (see Kaemmerer et al). 2003;126:1048-52

Hetzer R (see Koster et al). 2003;126:906-7 (Brief comm.)

Hibino N (see Isomatsu et al). 2003;126:1958-62 (Evolving tech.) 
Higaki N (see Higashiyama et al). 2003;126:611 (Letter reply) Higashiyama M, Kodama K, Takami K, Higaki N, Nakayama T, Yokouchi H. Malignant status at surgical margin of limitedresected non-small cell lung cancer: a crucial finding for predicting local relapse. 2003;126:611 (Letter reply)

Higuchi K, Koseni K, Takamoto S. Left-sided cervical aortic arch aneurysm: case report. 2003;126:2098-100 (Brief comm.)

Higuchi MdL (see Binotto et al). 2003;126:1026-32

Hijazi ZM (see Bacha et al). 2003;126:1718-23

Hill AC (see Wolf et al). 2003;126:168-78

Hillman ND (see Hawkins et al). 2003;126:247-53

Hirata M (see Bando et al). 2003;126:358-64

Hirata N, Asaoka N, Amemiya A, Hatsuoka S, Ueno T, Kosakai Y. Noninvasive evaluation of internal thoracic artery and left anterior descending coronary artery anastomotic sites using transthoracic Doppler echocardiography: comparison with coronary arteriography. 2003;126:1080-6

Hirata N (see Yoshitatsu et al). 2003;126:1531-6

Hirose H (see Mori et al). 2003;126:415-9

Hitchcock FJ (see Walhout et al). 2003;126:521-8

Ho Y-L (see Yu et al). 2003;126:80-9

Hofer A (see Mair et al). 2003;126:1378-84

Hofer C (see Genoni et al). 2003;126:288-9 (Brief comm.)

Hoffman GM (see Ghanayem et al). 2003;126:1367-77

Hoffmann H (see Pfannschmidt et al). 2003;126:732-9

Hoffmeier A (see Lunkenheimer et al). 2003;126:592-6 (Brief comm.)

Hofmann H (see Schoenburg et al). 2003;126:1455-60

Hofmann W (see Czerny et al). 2003;126:1296-301

Hogan PG, O'Brien MF. Improving the allograft valve: does the immune response matter? 2003;126:1251-3 (Editorial)

Holmes EC (see Samuels et al). 2003;126:2100-1 (Brief comm.)

Hopkins-Broyles D (see Olsen et al). 2003;126:992-9

Hornick L. Authors should list confounding factors and alternative explanations for adverse events seen with new technologies. 2003;126:1664 (Letter reply)

Horowitz JD (see Shah et al). 2003;126:1320-7

Horstkotte D. Problems with complication rate analysis. 2003;126: 1669-70 (Letter)

Hosaka S (see Osawa et al). 2003;126:604-5 (Brief comm.)

Houël R, Mazoyer E, Kirsch M, Boval B, Drouet L, Loisance DY. Resistance to aspirin after external ventricular assist device implantation. 2003;126:1636-7 (Brief comm.)

Hsu H-H (see Huang et al). 2003;126:2090-2 (Brief comm.)

Huang C-L (see Gotoh et al). 2003;126:1916-21

Huang P-M, Chen J-S, Hsu H-H, Chang Y-L, Chen C-W, Chien N-C, Lee Y-C. Staged dilation and stenting for long segmental tracheobronchial stenosis caused by tuberculosis. 2003;126: 2090-2 (Brief comm.)

Huang P-M (see Lee et al). 2003;126:298-9 (Brief comm.)

Huddleston C (see Lee et al). 2003;126:295-7 (Brief comm.)

Huddleston CB. Discussion of McMahon et al. 2003;126:1781-7

Huebner A (see Tossios et al). 2003;126:1513-20

Humpl T (see Adatia et al). 2003;126:1674-5 (Letter reply)

Hunt SA (see Demers et al). 2003;126:224-31

Hussain A (see Baslaim et al). 2003;126:586-8 (Brief comm.)
Hussain ST, Devagourou V, Kumar AS. Management of mitral paravalvular leak: therapy or misadventure? 2003;126:879-80 (Brief comm.)

Hutschala D (see Czerny et al). 2003;126:1296-301

Huynh TTT (see Estrera et al). 2003;126:1288-95

Hvass U, Baron F, Elsebaey A. A new aortoventriculoplasty for prosthetic aortic valve replacement. 2003;126:818-20

Hwang M-S, Chang Y-S, Chu J-J, Su W-J. Isolated persistent fifth aortic arch with systemic-to-pulmonary arterial connection. 2003;126:1643-4 (Brief comm.)

Hyman KM (see Bizekis et al). 2003;126:659-665. Correction 2003; $126: 1680$

Iacò AL (see Gaudino et al). 2003;126:1076-9

Iacoviello L (see Gaudino et al). 2003;126:1107-12

Iacovoni A (see Rescigno et al). 2003;126:1196-7 (Brief comm.)

Iannace E (see Carotti et al). 2003;126:1839-50

Ibrahim MF, Paparella D, Ivanov J, Buchanan MR, Brister SJ. Gender-related differences in morbidity and mortality during combined valve and coronary surgery. 2003;126:959-64

Ichimiya Y (see Watanabe et al). 2003;126:1212-4 (Brief comm.) Ichinoseki I (see Takahashi et al). 2003;126:600-2 (Brief comm.) Ignacio C (see Estrera et al). 2003;126:1288-95

Ignarro LJ (see Castellá et al). 2003;126:1442-8

Iivainen $\mathrm{T}$ (see $\mathrm{Wu}$ et al). 2003;126:1477-82

Iizasa T (see Chiyo et al). 2003;126:1141-6

Ikeda Y (see Aramaki et al). 2003;126:853-4 (Brief comm.)

Ikonomidis JS, Kratz JM, Crumbley AJ III, Stroud MR, Bradley SM, Sade RM, Crawford FA Jr. Twenty-year experience with the St Jude Medical mechanical valve prosthesis. 2003;126: 2022-31

Il'ina LN (see Pokrovsky et al). 2003;126:1071-5

Illiasch H (see Wandschneider and Illiasch). 2003;126:872-3 (Brief comm.)

Imahara SD (see Ad et al). 2003;126:1095-100

Imai Y (see Fischer et al). 2003;126:1174-80

Imamura H (see Uchiyama et al). 2003;126:148-59

Imanaka K, Kyo S, Asano H, Motomura N, Takamoto S, Kato M, Ogiwara M, Kohmoto O. Severe pulmonary stenosis and aortopulmonary fistula caused by a dissecting aneurysm in the ascending aorta. 2003;126:598-600 (Brief comm.)

Imanaka K (see Nishimura et al). 2003;126:1190-2 (Brief comm.)

Imano H (see Nakagawa et al). 2003;126:563-7

Imazuru T (see Fukuda and Imazuru). 2003;126:290-1 (Brief comm.)

Ino T (see Tanaka et al). 2003;126:837-41

Inoue H (see Naito et al). 2003;126:883-5 (Brief comm.)

Inoue M (see Okumura et al). 2003;126:1922-8

Irie Y (see Fischbein et al). 2003;126:216-23

Iserin F (see Boudjemline et al). 2003;126:490-7

Ishido H (see Senzaki et al). 2003;126:1645-7 (Brief comm.)

Ishii Y, Nitta T, Sakamoto S-i, Tanaka S, Asano G. Incisional atrial reentrant tachycardia: experimental study on the conduction property through the isthmus. 2003;126:254-62

Ishikawa M (see Nishimura et al). 2003;126:1190-2 (Brief comm.) Ishikawa S (see Raman et al). 2003;126:1357-66 
Ishino K, Sano S. Aortic arch repair with a working beating heart in premature infants. 2003;126:1653-4 (Brief comm.)

Ishino K (see Sano et al). 2003;126:504-10

Isomatsu Y, Shin'oka T, Matsumura G, Hibino N, Konuma T, Nagatsu M, Kurosawa H. Extracardiac total cavopulmonary connection using a tissue-engineered graft. 2003;126:1958-62 (Evolving tech.)

Israeli A (see Galai et al). 2003;126:1018-25

Ito H (see Gojo et al). 2003;126:885-6 (Brief comm.)

Itoyama Y (see Takahashi et al). 2003;126:1461-6

Ius P (see Bottio et al). 2003;126:99-105

Ivanov J (see David and Ivanov). 2003;126:939-41 (Editorial)

Ivanov J (see David et al). 2003;126:2118-9 (Letter reply)

Ivanov J (see Feindel et al). 2003;126:777-82

Ivanov J (see Ibrahim et al). 2003;126:959-64

Ivanov J (see Mickleborough et al). 2003;126:950-8

Iwakura A, Tabata Y, Koyama T, Doi K, Nishimura K, Kataoka K,

Fujita M, Komeda M. Gelatin sheet incorporating basic fibro-

blast growth factor enhances sternal healing after harvesting

bilateral internal thoracic arteries. 2003;126:1113-20

Iwanaka T (see Ko et al). 2003;126:897-9 (Brief comm.)

Iwata T (see Chiyo et al). 2003;126:1141-6

Iyoda A (see Chiyo et al). 2003;126:1141-6

Izumoto H (see Kawazoe et al). 2003;126:908-10 (Brief comm.)
Jonas RA, Wypij D, Roth SJ, Bellinger DC, Visconti KJ, du Plessis AJ, Goodkin H, Laussen PC, Farrell DM, Bartlett J, McGrath E, Rappaport LJ, Bacha EA, Forbess JM, del Nido PJ, Mayer JE Jr, Newburger JW. The influence of hemodilution on outcome after hypothermic cardiopulmonary bypass: results of a randomized trial in infants. 2003;126:1765-74

Jonas RA (see Bellinger et al). 2003;126:1385-96

Jonas RA (see Marshall et al). 2003;126:1427-33

Jonas RA (see Wypij et al). 2003;126:1397-403

Jonas RA. Discussion of McMahon et al. 2003;126:1781-7

Jones J (see Hawkins et al). 2003;126:247-53

Jones JM, Paxton LD, Graham ANJ. Acute postoperative lobar torsion associated with pulmonary arterial rupture. 2003;126: 303 (Letter)

Jones K. Discussion of Feindel et al. 2003;126:777-82

Jones K. Discussion of Lai et al. 2003;126:1978-86

Jonjev S, Schwertz DW, Beck JM, Ross JD, Law WR. Subcellular distribution of protein kinase $\mathrm{C}$ isozymes during cardioplegic arrest. 2003;126:1880-5

Joshi N (see Williams et al). 2003;126:436-41

Juhász-Nagy A (see Kollár et al). 2003;126:977-82

Juhász-Nagy A (see Szabó et al). 2003;126:651-8

Jung S-H (see Reed et al). 2003;126:1943-51

Juvonen T (see Kakko et al). 2003;126:106-13

\section{$J$}

Jäättelä M (see Frese et al). 2003;126:748-54

Jacobs ML. Discussion of Hawkins et al. 2003;126:247-53

Jacobs ML. Discussion of Monro et al. 2003;126:511-20

Jacobs S (see Falk et al). 2003;126:1575-9 (Evolving tech.)

Jahangiri M, Clarke J, Prefumo F, Pumphrey C, Ward D Cardiac surgery during pregnancy: pulsatile or nonpulsatile perfusion? 2003;126:894-895 (Brief comm.) Correction 2003;126:1680

Jahncke CL (see Kown et al). 2003;126:1065-70

Jamieson SW (see Chu et al). 2003;126:671-9

Jamieson SW (see Thistlethwaite and Jamieson). 2003;126:1228-9 (Letter reply)

Jamieson WRE (see Blackstone et al). 2003;126:783-96

Jamieson WRE. Discussion of Ikonomidis et al. 2003;126:2022-31

Jamjoom A (see Baslaim et al). 2003;126:586-8 (Brief comm.)

Jansen EW. Discussion of Wolf et al. 2003;126:168-78

Jaquis RDB (see Ghanayem et al). 2003;126:1367-77

Jarvik GP (see Gaynor et al). 2003;126:1736-45

Jassem J (see Rzyman et al). 2003;126:755-60

Jaubert F (see Boudjemline et al). 2003;126:490-7

Jeevanandam V (see Song et al). 2003;126:896-7 (Brief comm.)

Jenkins KJ (see Brown et al). 2003;126:272-81

Jenni R (see Kaemmerer et al). 2003;126:1048-52

Jessel A (see Boyce et al). 2003;126:420-7

Jessel A (see Gavard et al). 2003;126:807-13

Jessen ME (see Greilich et al). 2003;126:1498-503

Johannes F (see Cebi et al). 2003;126:2074-7 (Brief comm.)

John R (see Morgan et al). 2003;126:582-3 (Brief comm.)

John R (see Morgan et al). 2003;126:1624-33

Johnson JA (see Klena et al). 2003;126:857-9 (Brief comm.)

Johnston TS (see Trachiotis et al). 2003;126:2065-71

\section{K}

Kadota Y (see Okumura et al). 2003;126:1922-8

Kaemmerer H, Fratz S, Bauer U, Oechslin E, Brodherr-Heberlein S, Zrenner B, Turina J, Jenni R, Lange PE, Hess J. Emergency hospital admissions and three-year survival of adults with and without cardiovascular surgery for congenital cardiac disease. 2003; 126:1048-52

Kafi SA (see Wauthy et al). 2003;126:1434-41

Kaiser LR. Discussion of Absi et al. 2003;126:344-57

Kaiser LR. Discussion of Gonzalez-Stawinski et al. 2003;126: 1900-5

Kakko S, Räisänen T, Tamminen M, Airaksinen J, Groundstroem K, Juvonen T, Ylitalo A, Uusimaa P, Savolainen MJ. Candidate locus analysis of familial ascending aortic aneurysms and dissections confirms the linkage to the chromosome 5q13-14 in Finnish families. 2003;126:106-13

Kalantzi N (see Gerazounis et al). 2003;126:774-6

Kalil R (see Assad et al). 2003;126:300-2 (Brief comm.)

Kallenbach K (see Karck et al). 2003;126:602-4 (Brief comm.)

Kallenbach K (see Leyh et al). 2003;126:1000-4

Kaluza M (see Albes et al). 2003;126:1504-12

Kameyama K (see Gotoh et al). 2003;126:1916-21

Kameyama T (see Yamaguchi et al). 2003;126:870-2 (Brief comm.)

Kamikubo Y (see Murashita et al). 2003;126:1328-34

Kanaan SA (see Suda et al). 2003;126:1155-61

Kanaan SA (see Tagawa et al). 2003;126:1147-54

Kaneda Y (see Sugi et al). 2003;126:568-73

Kanemitsu S, Nishikawa M, Onoda K, Shimono T, Shimpo H, Yazaki A, Tanaka K, Shiku H, Yada I. Pharmacologic platelet anesthesia by glycoprotein IIb/IIIa complex antagonist and 
argatroban during in vitro extracorporeal circulation. 2003;126: 428-35

Kanter KR (see Dasika et al). 2003;126:2112 (Letter)

Kanter KR (see Trachiotis et al). 2003;126:2065-71

Karatzas D (see Paraskevaidis et al). 2003;126:913-4 (Brief comm.)

Karavas A (see Greelish et al). 2003;126:365-73

Karck M, Hagl C, Kallenbach K, Haverich A. The gut-first approach for repair of Crawford extent III thoracoabdominal aortic aneurysms. 2003;126:602-4 (Brief comm.)

Karliczek GF (see Schoenburg et al). 2003;126:1455-60

Karp RB. Discussion of Feindel et al. 2003;126:777-82

Kasahara S (see Sano et al). 2003;126:504-10

Kashem MA (see He et al). 2003;126:56-65

Kashima I (see Fukuda et al). 2003;126:1220-1 (Brief comm.)

Kasirajan K (see Dietl et al). 2003;126:1181-3 (Brief comm.)

Kasper JM (see Setser et al). 2003;126:48-55

Kasper S-M (see Tossios et al). 2003;126:1513-20

Kataoka K (see Iwakura et al). 2003;126:1113-20

Katariya K (see Bolooki et al). 2003;126:374-85

Katayose Y (see Nakagawa et al). 2003;126:563-7

Kato M (see Imanaka et al). 2003;126:598-600 (Brief comm.)

Kato M (see Nishimura et al). 2003;126:1190-2 (Brief comm.)

Kavukçu Ş (see Şahin et al). 2003;126:769-73

Kawada M (see Sano et al). 2003;126:504-10

Kawaharada N (see Yamada et al). 2003;126:597-8 (Brief comm.)

Kawahito K (see Tanaka et al). 2003;126:837-41

Kawara T (see Teshima et al). 2003;126:401-7

Kawashima H (see Ko et al). 2003;126:897-9 (Brief comm.)

Kawazoe K, Izumoto H, Tsuboi J, Koizumi J. Tricuspidization of incompetent bicuspid aortic valve. 2003;126:908-10 (Brief comm.)

Kaya B (see Sirlak et al). 2003;126:666-70

Kazemian P (see Fedak et al). 2003;126:797-806

Keeton BR (see Monro et al). 2003;126:511-20

Kékesi V (see Kollár et al). 2003;126:977-82

Kékesi V (see Szabó et al). 2003;126:651-8

Kereiakes D (see Boyce et al). 2003;126:420-7

Keresztes RS, Port JL, Pasmantier MW, Korst RJ, Altorki NK. Preoperative chemotherapy for esophageal cancer with paclitaxel and carboplatin: results of a phase II trial. 2003;126: 1603-8

Kernstine KH. Positron emission tomography with 2-[ $\left[{ }^{18} \mathrm{~F}\right]$ fluoro2-deoxy-D-glucose: can it be used to accurately stage the mediastinum in non-small cell lung cancer as an alternative to mediastinoscopy? 2003;126:1700-3 (Editorial)

Keshavjee S (see Fischer et al). 2003;126:1174-80

Khan MF (see Wimmer-Greinecker et al). 2003;126:465-8 (Evolving tech.)

Khan TA (see Ruel et al). 2003;126:1521-30

Khandwala F (see Koch et al). 2003;126:2032-43

Khandwala F (see Koch et al). 2003;126:2044-51

Khargi K (see Laczkovics et al). 2003;126:2119-20 (Letter)

Kherani AR, Cheema FH, Oz MC, Fal JM, Morgan JA, Topkara VK, Wilson DA, Vigilance DW, Garrido MJ, Naka Y. Implantation of a left ventricular assist device and the hub-and-spoke system in treating acute cardiogenic shock: who survives? 2003;126:1634-5 (Brief comm.)
Kherani AR (see Morgan et al). 2003;126:582-3 (Brief comm.) Kherani AR (see Morgan et al). 2003;126:1188-90 (Brief comm.) Kherani AR (see Morgan et al). 2003;126:1624-33

Khoury P (see Border et al). 2003;126:1760-4

Kido M (see Uchiyama et al). 2003;126:148-59

Kilo J (see Czerny et al). 2003;126:916-8 (Brief comm.)

Kim J-H (see Bizekis et al). 2003;126:659-665. Correction 2003; 126:1680

Kim L-H (see Svensson et al). 2003;126:892-3 (Brief comm.)

King MJ (see Beldholm et al). 2003;126:545-50

Kinouchi K (see Ko et al). 2003;126:897-9 (Brief comm.)

Kiovistoinen A (see Sihvo et al). 2003;126:1952-7

Kirsch M (see Houël). 2003;126:1636-7 (Brief comm.)

Kitahori K (see (Miyaji et al). 2003;126:1648-50 (Brief comm.)

Kitajima M (see Aramaki et al). 2003;126:853-4 (Brief comm.)

Kitamura N (see Yamagishi et al). 2003;126:1218-9 (Brief comm.)

Kitamura S (see Bando et al). 2003;126:358-64

Kitamura T, Motomura N, Ohtsuka T, Shibata K, Takayama H, Kotsuka Y, Takamoto S. Aortopulmonary fistula in pseudoaneurysm after ascending aortic surgery. 2003;126:904-5 (Brief comm.)

Kiziltepe U (see Sirlak et al). 2003;126:666-70

Klass O (see Tossios et al). 2003;126:1513-20

Kleber FX (see Oz et al). 2003;126:983-91

Klein P (see Borst et al). 2003;126:305 (Letter reply)

Klena JW, Danek SJ, Bostwick TK, Romero M, Johnson JA. Video-assisted thoracoscopic resection for intralobar pulmonary sequestration: single modality treatment with video-assisted thoracic surgery. 2003;126:857-9 (Brief comm.)

Klima U, Falk V, Maringka M, Bargenda S, Badack S, Moritz A, Mohr F, Haverich A, Wimmer-Greinecker G. Magnetic vascular coupling for distal anastomosis in coronary artery bypass grafting: a multicenter trial. 2003;126:1568-74 (Evolving tech.)

Kloevekorn WP (see Schoenburg et al). 2003;126:1455-60

Knight J (see Boyce et al). 2003;126:420-7

Ko L (see Verma et al). 2003;126:1555-60 (Evolving tech.)

Ko Y, Nakamura Y, Nomura K, Kinouchi K, Iwanaka T, Kawashima $\mathrm{H}$. Congenital thoracoabdominal aortic aneurysm. 2003;126:897-9 (Brief comm.)

Kobashigawa J (see Marelli et al). 2003;126:821-5

Kobayashi J (see Bando et al). 2003;126:358-64

Kobayashi T (see Senzaki et al). 2003;126:1645-7 (Brief comm.)

Koc H (see Reisli et al). 2003;126:584-5 (Brief comm.)

Koch CG, Khandwala F, Nussmeier N, Blackstone EH. Gender and outcomes after coronary artery bypass grafting: a propensity-matched comparison. 2003;126:2032-43

Koch CG, Khandwala F, Nussmeier N, Blackstone EH. Gender profiling in coronary artery bypass grafting. 2003;126:2044-51

Koch CG, Mora Mangano C, Schwann N, Vaccarino V. Is it gender, methodology, or something else? 2003;126:932-5 (Editorial)

Koch CG (see Vaccarino and Koch). 2003;126:1707-11 (Editorial)

Kodama K (see Higashiyama et al). 2003;126:611 (Letter reply)

Kofidis T, Balsam LB, Robbins RC. A few critical aspects-and Achilles heels- of tissue engineering approaches to restore injured myocardium. 2003;126:2113-4 (Letter)

Kohane IS (see Ruel et al). 2003;126:1521-30

Kohmoto O (see Imanaka et al). 2003;126:598-600 (Brief comm.) 
Koizumi J (see Kawazoe et al). 2003;126:908-10 (Brief comm.)

Koksal Y (see Reisli et al). 2003;126:584-5 (Brief comm.)

Kollár A, Kékesi V, Soós P, Juhász-Nagy A. Left ventricular external subannular plication: an indirect off-pump mitral annuloplasty method in a canine model. 2003;126:977-82

Komeda M (see Iwakura et al). 2003;126:1113-20

Komeda M (see Koyama et al). 2003;126:2110-1 (Letter reply)

Kondo H (see Nakagawa et al). 2003;126:1134-40

Kondo K (see Sakiyama et al). 2003;126:1207-9 (Brief comm.)

Kondo T (see Sugawara et al). 2003;126:1225 (Letter reply)

Konertz WF (see Oz et al). 2003;126:983-91

Konuma T (see Isomatsu et al). 2003;126:1958-62 (Evolving tech.)

Korst RJ (see Keresztes et al). 2003;126:1603-8

Korst RJ (see Mahtabifard et al). 2003;126:28-38

Korst RJ (see Merritt et al). 2003;126:1609-17

Kosakai Y (see Hirata et al). 2003;126:1080-6

Koseni K (see Higuchi et al). 2003;126:2098-100 (Brief comm.)

Koster A, Chew D, Kuebler W, Habazettl H, Hetzer R, Kuppe H. High antithrombin III levels attenuate hemostatic activation and leukocyte activation during cardiopulmonary bypass. 2003; 126:906-7 (Brief comm.)

Kotsuka Y (see Kitamura et al). 2003;126:904-5 (Brief comm.)

Kouatli A (see Baslaim et al). 2003;126:586-8 (Brief comm.)

Kouchoukos NT (see Okamoto et al). 2003;126:842-50

Kown MH, Lijkwan MA, Jahncke CL, Murata S, Rothbard JB, Robbins RC. L-Arginine polymers enhance coronary flow and reduce oxidative stress following cardiac transplantation in rats. 2003;126:1065-70

Kox WJ (see von Heymann et al). 2003;126:1667-8 (Letter)

Koyama K (see Gojo et al). 2003;126:885-6 (Brief comm.)

Koyama T, Nishimura K, Soga Y, Unimonh O, Komeda M. Apical versus basal partial ventriculectomy. 2003;126:2110-1 (Letter reply)

Koyama T (see Iwakura et al). 2003;126:1113-20

Kozower B, Meyers B. Lobar transplantation. 2003;126:2106 (Letter reply)

Kozower BD (see Suda et al). 2003;126:1155-61

Kozower BD (see Tagawa et al). 2003;126:1147-54

Krajisnik S (see Marchevsky et al). 2003;126:551-7

Krasna M. Discussion of Lerut et al. 2003;126:1121-8

Kratz JM (see Ikonomidis et al). 2003;126:2022-31

Kraus B (see Schoenburg et al). 2003;126:1455-60

Kremastinos DT (see Paraskevaidis et al). 2003;126:913-4 (Brief comm.)

Kresh JY (see Rangappa et al). 2003;126:124-32

Kresse H (see Fratz et al). 2003;126:1724-9

Kreuzer E (see Nollert et al). 2003;126:965-8

Krishnadasan B (see Naidu et al). 2003;126:200-7

Kuang J-Q (see Saito et al). 2003;126:114-23

Kucuker SA, Tasdemir O. Brachial artery cannulation. 2003;126: 2106-7 (Letter)

Kuebler W (see Koster et al). 2003;126:906-7 (Brief comm.)

Kuga T (see Takahashi et al). 2003;126:600-2 (Brief comm.)

Kuipers I (see Schoof et al). 2003;126:282-3 (Brief comm.)

Kumar AS. Free right atrial patches for septal defect closure. 2003;126:303-4 (Letter)
Kumar AS. Medical resources and capital punishment. 2003;126: 2107-8 (Letter)

Kumar AS (see Hussain et al). 2003;126:879-80 (Brief comm.)

Kuppe H (see Koster et al). 2003;126:906-7 (Brief comm.)

Kurlansky PA. Is there a hypercoagulable state after off-pump coronary artery bypass surgery? What do we know and what can we do? 2003;126:7-10 (Editorial)

Kurlansky PA. Discussion of Damiano et al. 2003;126:2016-21

Kurosawa H (see Isomatsu et al). 2003;126:1958-62 (Evolving tech.)

Kuster J-R (see Frese et al). 2003;126:748-54

Kutlay H (see Şahin et al). 2003;126:769-73

Kwa HB (see van der Maten et al). 2003;126:740-3

Kyo S (see Gojo et al). 2003;126:885-6 (Brief comm.)

Kyo S (see Imanaka et al). 2003;126:598-600 (Brief comm.)

Kyo S (see Nishimura et al). 2003;126:1190-2 (Brief comm.)

Kyo S (see Senzaki et al). 2003;126:1645-7 (Brief comm.)

\section{$\mathrm{L}$}

La Canna G (see Maisano et al). 2003;126:1987-97

Lacasse Y, Plante J, Martel S, Raby B. Transthoracic needle biopsy in the diagnosis of solitary pulmonary nodules: a survey of Canadian physicians. 2003;126:761-8

Lackey S (see Ardehali et al). 2003;126:1929-34

Lacour-Gayet F. Discussion of Sano et al. 2003;126:504-10

Laczkovics A, Khargi K, Deneke T. Esophageal perforation during left atrial radiofrequency ablation. 2003;126:2119-20 (Letter)

Lai DT, Miller DC, Mitchell RS, Oyer PE, Moore KA, Robbins RC, Shumway NE, Reitz BA. Acute type A aortic dissection complicated by aortic regurgitation: composite valve graft versus separate valve graft versus conservative valve repair. 2003; 126:1978-86

Laine GA (see Fischer et al). 2003;126:1483-8

Laks H (see Ardehali et al). 2003;126:1929-34

Laks H (see Fischbein et al). 2003;126:216-23

Laks H (see Gupta et al). 2003;126:1746-52

Laks H (see Marelli et al). 2003;126:821-5

Laks H. Discussion of Greelish et al. 2003;126:365-73

Lalezari S, Hazekamp MG, Bartelings MM, Schoof PH, Gittenberger-de Groot AC. Pulmonary artery remodeling in transposition of the great arteries: relevance for neoaortic root dilatation. 2003;126:1053-60

Lam B-K, Pettersson GB, Vogt DP. Urgent inferior vena cava replacement with an autologous pericardium tube graft. 2003; 126:2101-3 (Brief comm.)

Lam J (see Schoof et al). 2003;126:282-3 (Brief comm.)

Lambert LM (see Hawkins et al). 2003;126:247-53

Lammer J (see Czerny et al). 2003;126:916-8 (Brief comm.)

Lamper C (see Goldberg et al). 2003;126:1168-73

Landolfo KP (see Scarborough et al). 2003;126:1561-7 (Evolving tech.)

Landoni C (see Pastorino et al). 2003;126:1906-10

Lang P (see Marshall et al). 2003;126:1427-33

Lange PE (see Kaemmerer et al). 2003;126:1048-52

Langer F, Wilhelm W, Tscholl D, Schramm R, Lausberg H, Wendler O, Schäfers H-J. Intraoperative inhalation of the long- 
acting prostacyclin analog iloprost for pulmonary hypertension. 2003;126:874-5 (Brief comm.)

Lansman SL (see Hagl et al). 2003;126:1005-12

Lapenna E (see Alfieri et al). 2003;126:75-9

Lara PN (see Roberts et al). 2003;126:1597-602

Large SR (see Stoica et al). 2003;126:2072-4 (Brief comm.)

Larson SM (see Flores et al). 2003;126:11-6

Lass M (see Oz et al). 2003;126:983-91

Latson LA (see Duncan et al). 2003;126:694-702

Lattouf OM (see Corvera et al). 2003;126:1549-54

Lau CL (see Force et al). 2003;126:864-6 (Brief comm.)

Lau DH (see Roberts et al). 2003;126:1597-602

Laufer G (see Hangler et al). 2003;126:391-400

Laurikka J (see Wu et al). 2003;126:1477-82

Lausberg H (see Langer et al). 2003;126:874-5 (Brief comm.)

Laussen PC (see Jonas et al). 2003;126:1765-74

Lauwers P (see Sergeant et al). 2003;126:1279-87

Law S (see Murthy et al). 2003;126:1162-7

Law WR (see Jonjev et al). 2003;126:1880-5

Lawton JS, Brister SJ, Petro KR, Dullum M. Surgical revascularization in women: unique intraoperative factors and considerations. 2003;126:936-8 (Editorial)

Lawton JS (see Moazami et al). 2003;126:2097-8 (Brief comm.)

Lawton JS (see Olsen et al). 2003;126:992-9

Leacche M (see Greelish et al). 2003;126:365-73

Lebouitz K (see Bonanomi et al). 2003;126:851-2 (Evolving tech.)

Lechner E (see Mair et al). 2003;126:1378-84

Lee CN (see El Oakley et al). 2003;126:1668 (Letter)

Lee JK (see Absi et al). 2003;126:344-57

Lee R, Mendeloff EN, Huddleston C, Sweet SC, de la Morena M. Bilateral lung transplantation for pulmonary hypoplasia caused by congenital diaphragmatic hernia. 2003;126:295-7 (Brief comm.)

Lee RMKW (see Gao et al). 2003;126:179-85

Lee Y-C, Chang Y-L, Chen C-W, Chien N-C, Huang P-M. Use of autologous pleural flap buttress in thoracoscopic lung volume reduction surgery. 2003;126:298-9 (Brief comm.)

Lee Y-C (see Huang et al). 2003;126:2090-2 (Brief comm.)

Leeuwenburgh BPJ, Schoof PH, Steendijk P, Baan J, Mooi WJ, Helbing WA. Chronic and adjustable pulmonary artery banding: reflections on old knowledge. 2003;126:2105-6 (Letter reply)

Lekkerkerker JC (see Walhout et al). 2003;126:521-8

Lemaire A (see Gonzalez-Stawinski et al). 2003;126:1900-5

Lemercier E (see Litzler et al). 2003;126:1204-7 (Brief comm.)

Lemmer JH Jr (see Blackstone et al). 2003;126:783-96

Lemmon J (see Saxena et al). 2003;126:90-8

Leo F (see Pastorino et al). 2003;126:1906-10

Leon M (see Pastorino et al). 2003;126:1906-10

Leon ME (see Veronesi et al). 2003;126:1670-1 (Letter)

Lerut T, Coosemans W, Decker G, De Leyn P, Ectors N, Fieuws S, Moons J, Nafteux P, Van Raemdonck D, Leuven Collaborative Workgroup for Esophageal Carcinoma. Extracapsular lymph node involvement is a negative prognostic factor in T3 adenocarcinoma of the distal esophagus and gastroesophageal junction. 2003;126:1121-8

Lesèche G (see Duariat et al). 2003;126:875-7 (Brief comm.)

Lesèche G (see Thabut et al). 2003;126:469-75
Leuven Collaborative Workgroup for Esophageal Carcinoma (see Lerut et al). 2003;126:1121-8

Levi D (see Gupta et al). 2003;126:1746-52

Levin LS (see Domkowski et al). 2003;126:386-90

Levine M (see Ardehali et al). 2003;126:1929-34

Lewin M (see Lupinetti et al). 2003;126:240-6

Leyh RG, Wilhelmi M, Walles T, Kallenbach K, Rebe P, Oberbeck A, Herden T, Haverich A, Mertsching H. Acellularized porcine heart valve scaffolds for heart valve tissue engineering and the risk of cross-species transmission of porcine endogenous retrovirus. 2003;126:1000-4

Li R-K (see Fazel et al). 2003;126:2114-6 (Letter reply)

Li R-K (see Ohno et al). 2003;126:1537-48

Li R-K (see Verma et al). 2003;126:1886-91

Li S-H (see Verma et al). 2003;126:1886-91

Libermann TA (see Ruel et al). 2003;126:1521-30

Liddicoat JR (see Ruel et al). 2003;126:1521-30

Lieber ML (see Setser et al). 2003;126:48-55

Lien D (see McNamee et al). 2003;126:861-3 (Brief comm.)

Lien D (see McNamee et al). 2003;126:910-2 (Brief comm.)

Lijkwan MA (see Kown et al). 2003;126:1065-70

Likungu JA (see Schmitz et al). 2003;126:645-50

Lila N (see Zegdi et al). 2003;126:1867-74

Lim E, Motalleb-Zadeh R, Wallard M, Misra N, Akowauh E, Ahmed I, Halstead JC, Murphy F, Foweraker J, Tsui S. Pyrexia after cardiac surgery: natural history and association with infection. 2003;126:1013-7

Lim K (see Caputo et al). 2003;126:39-47

Lima G (see Assad et al). 2003;126:300-2 (Brief comm.)

Lin BJ (see McClurken et al). 2003;126:293-5 (Brief comm.)

Lin CCH (see Saito et al). 2003;126:114-23

Lin F-Y (see Yu et al). 2003;126:80-9

Lin M (see Wypij et al). 2003;126:1397-403

Lin T-Y, Chiu K-M, Wang M-J, Chu S-H. Carbon dioxide embolism during endoscopic saphenous vein harvesting in coronary artery bypass surgery. 2003;126:2011-5

Litwin SB (see Ghanayem et al). 2003;126:1367-77

Litwin SB. Discussion of Sano et al. 2003;126:504-10

Litzler P-Y, Douvrin F, Bouchart F, Tabley A, Lemercier E, Baste J-M, Redonnet M, Haas-Hubscher C, Clavier E, Bessou J-P. Combined endovascular and video-assisted thoracoscopic procedure for treatment of a ruptured pulmonary arteriovenous fistula: case report and review of the literature. 2003;126: 1204-7 (Brief comm.)

Liu D (see Gotoh et al). 2003;126:1916-21

Liu M (see Fischer et al). 2003;126:1174-80

Lock JE (see Marshall et al). 2003;126:1427-33

Lock-Buckley P (see Olsen et al). 2003;126:992-9

LoForte A (see Chiappini et al). 2003;126:1788-91

Loisance DY (see Houël). 2003;126:1636-7 (Brief comm.)

Long JW. Discussion of Domkowski et al. 2003;126:386-90

Lorini L (see Rescigno et al). 2003;126:1196-7 (Brief comm.)

Loubani M, Ghosh S, Galiñanes M. The aging human myocardium: tolerance to ischemia and responsiveness to ischemic preconditioning. 2003;126:143-7

Love BA (see Marshall et al). 2003;126:1427-33

Luketich JD. Discussion of Lerut et al. 2003;126:1121-8

Lundell DC (see Wolf et al). 2003;126:168-78 
Lunkenheimer P. The helix and the heart. 2003;126:920-1 (Letter)

Lunkenheimer PP, Anderson RH. Apical versus basal partial ventriculectomy. 2003;126:2109-10 (Letter)

Lunkenheimer PP, Redmann K, Florek JC, Scheld HH, Hoffmeier A, Cryer CW, Batista RV, Stanton JJ, Filho JDF, Anderson $\mathrm{RH}$. Surgical reduction of ventricular radius by aspirated plication of the myocardial wall: an experimental study. 2003; 126:592-6 (Brief comm.)

Lupinetti FM, Duncan BW, Lewin M, Dyamenahalli U, Rosenthal GL. Comparison of autograft and allograft aortic valve replacement in children. 2003;126:240-6

Lutz J (see Trachiotis et al). 2003;126:2065-71

Lyon H (see Ruel et al). 2003;126:1521-30

Lytle BW. Radial versus right internal thoracic artery as a second arterial conduit for coronary surgery: early and midterm outcomes. 2003;126:5-6 (Editorial)

Lytle BW (see Gillinov et al). 2003;126:1661-2 (Letter)

Lytle BW (see Svensson et al). 2003;126:892-3 (Brief comm.)

\section{M}

Ma Y (see DiPerna et al). 2003;126:1129-33

Mabuchi M (see Murashita et al). 2003;126:1328-34

MacApinlac HA (see Reed et al). 2003;126:1943-51

Macchiarini P (see Go et al). 2003;126:867-9 (Brief comm.)

Mack M. Discussion of Schmitz et al. 2003;126:1829-38

MacLean AA (see Fischer et al). 2003;126:1174-80

Madan N, Gaynor JW, Tanel R, Cohen M, Nicholson S, Vetter V, Rhodes L. Single-finger subcutaneous defibrillation lead and "active can": a novel minimally invasive defibrillation configuration for implantable cardioverter-defibrillator implantation in a young child. 2003;126:1657-9 (Brief comm.)

Madhavan A, Goodfellow J. Lack of evidence for vitamin C as acute vasodilator. 2003;126:1671-2 (Letter)

Madrigal L (see Hernández et al). 2003;126:2081-2 (Brief comm.)

Maeda H (see Ohta et al). 2003;126:1671 (Letter reply)

Maehara Y (see Yamaguchi et al). 2003;126:870-2 (Brief comm.)

Maeshima A (see Nakagawa et al). 2003;126:1134-40

Maestri R (see Colli et al). 2003;126:881-3 (Brief comm.)

Maganti M (see Davierwala et al). 2003;126:1335-44

Mahesh B (see Caputo et al). 2003;126:39-47

Mahnke B (see Pigula et al). 2003;126:1650-2 (Brief comm.)

Mahtabifard A, Merritt RE, Yamad RE, Crystal RG, Korst RJ. In vivo gene transfer of pigment epithelium-derived factor inhibits tumor growth in syngeneic murine models of thoracic malignancies. 2003;126:28-38

Mahtabifard A (see Merritt et al). 2003;126:1609-17

Mair R, Tulzer G, Sames E, Gitter R, Lechner E, Steiner J, Hofer A, Geiselseder G, Gross C. Right ventricular to pulmonary artery conduit instead of modified Blalock-Taussig shunt improves postoperative hemodynamics in newborns after the Norwood operation. 2003;126:1378-84

Maisano F, Caldarola A, Blasio A, De Bonis M, La Canna G, Alfieri O. Midterm results of edge-to-edge mitral valve repair without annuloplasty. 2003;126:1987-97

Maisano F (see Alfieri et al). 2003;126:75-9
Maish M, Vaporciyan AA. Chondrosarcoma arising in the trachea: a case report and review of the literature. 2003;126:2077-80 (Brief comm.)

Makino Y (see Murashita et al). 2003;126:1328-34

Mal H (see Duariat et al). 2003;126:875-7 (Brief comm.)

Mal H (see Thabut et al). 2003;126:469-75

Malas M (see Wells et al). 2003;126:498-503

Mallidi HR, Fremes SE. Study of warm perfusion rather than cardioplegia. 2003;126:2111-2 (Letter reply)

Mallon SM (see Bolooki et al). 2003;126:374-85

Mancini D (see Williams et al). 2003;126:436-41

Mancini DM (see Bisleri et al). 2003;126:1639-40 (Brief comm.)

Mancini DM (see Morgan et al). 2003;126:1624-33

Mancuso M (see Filosso et al). 2003;126:1215-7 (Brief comm.)

Mangano CM (see Koch et al). 2003;126:932-5 (Editorial)

Maniar HS (see Prasad et al). 2003;126:1822-8

Manning PB (see Border et al). 2003;126:1760-4

Marchetto G (see Caputo et al). 2003;126:39-47

Marchevsky AM, Qiao J-H, Krajisnik S, Mirocha JM, McKenna RJ. The prognostic significance of intranodal isolated tumor cells and micrometastases in patients with non-small cell carcinoma of the lung. 2003;126:551-7

Marcial MB (see Assad et al). 2003;126:300-2 (Brief comm.)

Marczin N. Exhaled nitric oxide and cardiac surgery with extracorporeal circulation. 2003;126:1673-4 (Letter)

Marelli D, Laks H, Bresson S, Ardehali A, Bresson J, Esmailian F, Plunkett M, Moriguchi J, Kobashigawa J. Results after transplantation using donor hearts with preexisting coronary artery disease. 2003;126:821-5

Margaritora S, Cesario A, Granone P. Pattern of lymphatic spread and prognosis of $\mathrm{pN} 1$ non-small cell lung cancer: what does it stand for? 2003;126:1664-5 (Letter)

Marinelli G (see Chiappini et al). 2003;126:1788-91

Maringka M (see Klima et al). 2003;126:1568-74 (Evolving tech.)

Marino B, Corno AF. Spiral pattern: universe, normal heart, and complex congenital defects. 2003;126:1225-6 (Letter)

Marino B (see Carotti et al). 2003;126:1666-7 (Letter)

Marra A, Stamatis G. Pattern of lymphatic spread and prognosis of pN1 non-small cell lung cancer: what does it stand for? 2003; 126:1665-6 (Letter reply)

Marrash-Chahla R (see Thabut et al). 2003;126:469-75

Marshall AC, Love BA, Lang P, Jonas RA, del Nido PJ, Mayer JE, Lock JE. Staged repair of tetralogy of Fallot and diminutive pulmonary arteries with a fenestrated ventricular septal defect patch. 2003;126:1427-33

Martel S (see Lacasse et al). 2003;126:761-8

Martens S (see Doss et al). 2003;126:814-7

Martin U (see Petersen et al). 2003;126:2086-90 (Brief comm.)

Martìn-Suàrez S (see Chiappini et al). 2003;126:1788-91

Maseri A (see Gaudino et al). 2003;126:1107-12

Massad M (see Shuhaiber et al). 2003;126:1660-1 (Letter)

Massih TA (see Boudjemline et al). 2003;126:490-7

Masuda Z-i (see Sano et al). 2003;126:504-10

Masuoka A (see Nishimura et al). 2003;126:1190-2 (Brief comm.)

Masutani S (see Senzaki et al). 2003;126:1645-7 (Brief comm.)

Mateos M (see Oliver et al). 2003;126:711-7

Mathew JP (see Scarborough et al). 2003;126:1561-7 (Evolving tech.) 
Mathieu P, Roussel JC, Dagenais F, Anegon I. Cartilaginous metaplasia and calcification in aortic allograft is associated with transforming growth factor $\beta 1$ expression. 2003; $126: 1449-54$

Mathisen DJ (see Gaissert et al). 2003;126:744-7

Mathisen DJ. Discussion of DeCamp et al. 2003;126:17-27

Mathisen DJ. Discussion of Ferguson. 2003;126:1935-42

Matsubara H (see Date et al). 2003;126:476-81

Matsuda H (see Hayashi et al). 2003;126:1813-21

Matsuda H (see Ohta et al). 2003;126:1671 (Letter reply)

Matsuda H (see Okumura et al). 2003;126:1922-8

Matsuda T (see $\mathrm{He}$ et al). 2003;126:455-64

Matsui H (see (Miyaji et al). 2003;126:1648-50 (Brief comm.)

Matsumura G (see Isomatsu et al). 2003;126:1958-62 (Evolving tech.)

Matsuno Y (see Mori et al). 2003;126:415-9

Matsuno Y (see Nakagawa et al). 2003;126:1134-40

Matsuoka H (see Sakiyama et al). 2003;126:1207-9 (Brief comm.)

Matsushita T (see Yamagishi et al). 2003;126:1218-9 (Brief comm.)

Matteucci MLS (see Rescigno et al). 2003;126:1196-7 (Brief comm.)

Mattioli S (see Pacini et al). 2003;126:900-2 (Brief comm.)

Matunaga T (see Senzaki et al). 2003;126:1645-7 (Brief comm.)

Mawatari T (see Watanabe et al). 2003;126:1212-4 (Brief comm.)

Mayer JE Jr (see Jonas et al). 2003;126:1765-74

Mayer JE Jr (see Marshall et al). 2003;126:1427-33

Mayer JE Jr. Discussion of Hawkins et al. 2003;126:247-53

Mazoyer E (see Houël). 2003;126:1636-7 (Brief comm.)

McCarthy PM. Ventricular aneurysms, shock, and late follow-up in patients with heart failure. 2003;126:323-5 (Editorial)

McCarthy PM (see Gillinov et al). 2003;126:1661-2 (Letter)

McCarthy PM (see Setser et al). 2003;126:48-55

McClurken JB, Hammer WJ, Lin BJ. Transcardiac gunshot wound recognized forty-eight years later. 2003;126:293-5 (Brief comm.)

McCrindle BW (see van Dongen et al). 2003;126:703-10

McDonald-McGinn D (see Gaynor et al). 2003;126:1736-45

McGowan FX (see Friehs et al). 2003;126:263-71

McGrath E (see Jonas et al). 2003;126:1765-74

McKenna RJ (see Marchevsky et al). 2003;126:551-7

McKenzie ED (see Andropoulos et al). 2003;126:1712-7

McKneally M. Cardiac transplantation in prisoners. 2003;126: 1227 (Letter reply)

McKneally M. Medical resources and capital punishment. 2003; 126:2108 (Letter reply)

McLean JE (see Yarbrough et al). 2003;126:1489-97

McLean JE (see Yarbrough et al). 2003;126:1892-9

McMahon A-M, van Doorn C, Burch M, Whitmore P, Neligan S, Rees P, Radley-Smith R, Goldman A, Brown K, Cohen G, Tsang V, Elliott M, de Leval MR. Improved early outcome for end-stage dilated cardiomyopathy in children. 2003; $126: 1781-7$

McNamee CJ, Lien D, Puttagunta L, Conlan AA. Solitary squamous papillomas of the bronchus: a rare case report and literature review. 2003;126:861-3 (Brief comm.)
McNamee CJ, Modry DL, Lien D, Conlan AA. Drowned donor lung for bilateral lung transplantation. 2003;126:910-2 (Brief comm.)

McNeil K (see Stoica et al). 2003;126:2072-4 (Brief comm.)

Mecozzi G, Pratali S, Milano A, Nardi C, Bortolotti U. Severe quadricuspid aortic valve stenosis after mediastinal irradiation. 2003;126:1198-9 (Brief comm.)

Mecozzi G (see De Carlo et al). 2003;126:337-43

Medalion B, Frenkel G, Patachenko P, Hauptman E, Sasson L, Schachner A. Preoperative use of enoxaparin is not a risk factor for postoperative bleeding after coronary artery bypass surgery. 2003;126:1875-9

Mee RBB (see Duncan et al). 2003;126:694-702

Mehlhorn U (see Fischer et al). 2003;126:1483-8

Mehlhorn U (see Tossios et al). 2003;126:1513-20

Meijboom EJ (see Walhout et al). 2003;126:521-8

Meijer R (see Borst et al). 2003;126:305 (Letter reply)

Meiser B (see Gulbins et al). 2003;126:890-1 (Brief comm.)

Melo JQ, Benussi S, Tortoriello W, Santinelli V, Calvi S, Nascimbene S, Pappone C, Alfieri O. Origin of atrial fibrillation from the pulmonary veins in a mitral patient. 2003;126:914-6 (Brief comm.)

Melvin D (see He et al). 2003;126:56-65

Mendeloff EN (see Lee et al). 2003;126:295-7 (Brief comm.)

Mentzer RM Jr. Does size matter? What is your infarct rate after coronary artery bypass grafting? 2003;126:326-8 (Editorial)

Mentzer RM Jr (see Vinten-Johansen and Mentzer). 2003;126: 1265-7 (Editorial)

Mentzer SJ. Discussion of Okumura et al. 2003;126:1922-8

Mentzer SJ. Discussion of Sihvo et al. 2003;126:1952-7

Meo F (see Carotti et al). 2003;126:1839-50

Merchant F (see Gonzalez-Stawinski et al). 2003;126:1900-5

Merrick S. Discussion of Greelish et al. 2003;126:365-73

Merritt RE, Mahtabifard A, Yamada RE, Crystal RG, Korst RJ. Cisplatin augments cytotoxic T-lymphocyte-mediated antitumor immunity in poorly immunogenic murine lung cancer. 2003; $126: 1609-17$

Merritt RE (see Mahtabifard et al). 2003;126:28-38

Mertsching H (see Leyh et al). 2003;126:1000-4

Mesia CI (see Duncan et al). 2003;126:694-702

Metzdorff M. Discussion of Lupinetti et al. 2003;126:240-6

Meyer A (see Fischer et al). 2003;126:1641-3 (Brief comm.)

Meyers B (see Kozower and Meyers). 2003;126:2106 (Letter reply)

Meyers BF (see Reed et al). 2003;126:1943-51

Meyns B (see Sergeant et al). 2003;126:1279-87

Michelfelder EC (see Border et al). 2003;126:1760-4

Mickle DAG (see Ohno et al). 2003;126:1537-48

Mickle DAG (see Verma et al). 2003;126:1886-91

Mickleborough LL, Carson S, Ivanov J. Gender differences in quality of distal vessels: effect on results of coronary artery bypass grafting. 2003;126:950-8

Mickleborough LL (see Hartz et al). 2003;126:620-2 (Editorial)

Mierdl S (see Wimmer-Greinecker et al). 2003;126:465-8 (Evolving tech.)

Miescher D (see Frese et al). 2003;126:748-54

Migliavacca F (see Bove et al). 2003;126:1040-7 
Migliore M. Efficacy and safety of single-trocar technique for minimally invasive surgery of the chest in the treatment of noncomplex pleural disease. 2003;126:1618-23

Mignatti P (see Bizekis et al). 2003;126:659-665. Correction 2003; 126:1680

Miksch J (see Nollert et al). 2003;126:965-8

Milano A (see Mecozzi et al). 2003;126:1198-9 (Brief comm.)

Milano AD (see De Carlo et al). 2003;126:337-43

Milano CA (see Petrofski et al). 2003;126:442-7

Milano CA. Discussion of Verma et al. 2003;126:1886-91

Mildner RJ (see van Dongen et al). 2003;126:703-10

Miller CC III (see Estrera et al). 2003;126:1288-95

Miller D. Discussion of Flores et al. 2003;126:11-6

Miller DC (see Blackstone et al). 2003;126:783-96

Miller DC (see Lai et al). 2003;126:1978-86

Miller DC. Discussion of Farivar and Cohn. 2003;126:969-76

Miller DC. Discussion of Sergeant et al. 2003;126:1279-87

Minamiya Y (see Nakagawa et al). 2003;126:563-7

Minamiya Y (see Saito et al). 2003;126:609 (Letter reply)

Mingoia JT (see Yarbrough et al). 2003;126:1489-97

Mingoia JT (see Yarbrough et al). 2003;126:1892-9

Minich LL (see Hawkins et al). 2003;126:247-53

Mirocha JM (see Marchevsky et al). 2003;126:551-7

Misra N (see Lim et al). 2003;126:1013-7

Mitchell M (see Greelish et al). 2003;126:365-73

Mitchell RS (see Lai et al). 2003;126:1978-86

Mitsuno M (see Yoshitatsu et al). 2003;126:1531-6

Miyaji K, Nagata N, Matsui H, Miyamoto T, Kitahori K. Successful Fontan procedure for asplenia with pulmonary atresia and major aortopulmonary collateral arteries. 2003;126:1648-50 (Brief comm.)

Miyamoto T (see (Miyaji et al). 2003;126:1648-50 (Brief comm.)

Miyamoto Y (see Hayashi et al). 2003;126:1813-21

Miyamoto Y (see Yoshitatsu et al). 2003;126:1531-6

Miyaoka E (see Nakagawa et al). 2003;126:1134-40

Miyazaki T (see Yamagishi et al). 2003;126:1218-9 (Brief comm.)

Miyoshi S (see Okumura et al). 2003;126:1922-8

Miyoshi T (see Sakiyama et al). 2003;126:1207-9 (Brief comm.)

Mizutani E (see Naito et al). 2003;126:883-5 (Brief comm.)

Moazami N, Moon MR, Lawton JS, Bailey M, Damiano R Jr. Axillary artery cannulation for extracorporeal membrane oxygenator support in adults: an approach to minimize complications. 2003;126:2097-8 (Brief comm.)

Moazami N (see Force et al). 2003;126:864-6 (Brief comm.)

Modry DL (see McNamee et al). 2003;126:910-2 (Brief comm.)

Moffatt S (see Demers et al). 2003;126:224-31

Mohanakumar T (see Suda et al). 2003;126:1155-61

Mohr F (see Klima et al). 2003;126:1568-74 (Evolving tech.)

Mohr FW (see Doll et al). 2003;126:1662 (Letter reply)

Mohr FW (see Doll et al). 2003;126:1663 (Letter reply)

Mohr FW (see Doll et al). 2003;126:2120 (Letter reply)

Mohr FW (see Falk et al). 2003;126:1575-9 (Evolving tech.)

Mohr FW (see Oz et al). 2003;126:983-91

Mohr FW. Discussion of Wolf et al. 2003;126:168-78

Monden Y (see Sakiyama et al). 2003;126:1207-9 (Brief comm.)

Monro JL, Alexiou C, Salmon AP, Keeton BR. Reoperations and survival after primary repair of congenital heart defects in children. 2003;126:511-20
Mooi WJ (see Leeuwenburgh et al). 2003;126:2105-6 (Letter reply)

Mooi WJ (see Wauthy et al). 2003;126:1434-41

Moon M (see Absi et al). 2003;126:344-57

Moon MR (see Moazami et al). 2003;126:2097-8 (Brief comm.)

Moon MR (see Okamoto et al). 2003;126:842-50

Moons J (see Lerut et al). 2003;126:1121-8

Moore KA (see Lai et al). 2003;126:1978-86

Morales DL (see Morgan et al). 2003;126:582-3 (Brief comm.)

Moran AM (see Brown et al). 2003;126:272-81

Morgan JA, John R, Weinberg AD, Kherani AR, Colletti NJ, Vigilance DW, Cheema FH, Bisleri G, Cosola T, Mancini DM, $\mathrm{Oz}$ MC, Edwards NM. Prolonged donor ischemic time does not adversely affect long-term survival in adult patients undergoing cardiac transplantation. 2003;126:1624-33

Morgan JA, Morales DL, John R, Ginsburg ME, Kherani AR, Vigilance DW, Cheema FH, Smith CR Jr, Oz MC, Argenziano M. Endoscopic, robotically assisted implantation of phrenic pacemakers. 2003;126:582-3 (Brief comm.)

Morgan JA, Park YK, Kherani AR, Vigilance DW, Cheema FH, $\mathrm{Oz} \mathrm{MC,} \mathrm{Naka} \mathrm{Y.} \mathrm{Does} \mathrm{bridging} \mathrm{to} \mathrm{transplantation} \mathrm{with} \mathrm{a} \mathrm{left}$ ventricular assist device adversely affect posttransplantation survival? A comparative analysis of mechanical versus inotropic support. 2003;126:1188-90 (Brief comm.)

Morgan JA (see Bisleri et al). 2003;126:1639-40 (Brief comm.)

Morgan JA (see Kherani et al). 2003;126:1634-5 (Brief comm.)

Mori Y, Hirose H, Takagi H, Umeda Y, Fukumoto Y, Shimabukuro K, Matsuno Y. Aortic arch repair for Stanford type A aortic dissection with distal anastomosis to the proximal level of the distal aortic arch. 2003;126:415-9

Moriguchi J (see Marelli et al). 2003;126:821-5

Morishita K (see Yamada et al). 2003;126:597-8 (Brief comm.)

Moritz A (see Doss et al). 2003;126:814-7

Moritz A (see Klima et al). 2003;126:1568-74 (Evolving tech.)

Moritz A (see Wimmer-Greinecker et al). 2003;126:465-8 (Evolving tech.)

Morris CD (see Corvera et al). 2003;126:1549-54

Morton JR. Discussion of Sergeant et al. 2003;126:1279-87

Morton LS (see Omar et al). 2003;126:832-6

Moss A (see Harrington et al). 2003;126:638-44

Motalleb-Zadeh R (see Lim et al). 2003;126:1013-7

Motomura N (see Imanaka et al). 2003;126:598-600 (Brief comm.)

Motomura N (see Kitamura et al). 2003;126:904-5 (Brief comm.)

Moustardas M (see Gerazounis et al). 2003;126:774-6

Muehling A (see Schoenburg et al). 2003;126:1455-60

Mueller L (see Hangler et al). 2003;126:391-400

Mukherjee R (see Yarbrough et al). 2003;126:1489-97

Mukherjee R (see Yarbrough et al). 2003;126:1892-9

Mulder D (see Ferri et al). 2003;126:877-9 (Brief comm.)

Muley T (see Pfannschmidt et al). 2003;126:732-9

Mulligan MS (see Naidu et al). 2003;126:200-7

Mulligan MS (see Naidu et al). 2003;126:482-9

Mulvihill DM (see Bradley et al). 2003;126:1033-9

Mulvihill DM (see Simsic et al). 2003;126:186-90

Munakata M (see Takahashi et al). 2003;126:600-2 (Brief comm.)

Munoz R (see Pigula et al). 2003;126:1650-2 (Brief comm.)

Murad S (see Omar et al). 2003;126:832-6

Murakami H (see Yoshimura et al). 2003;126:1417-26 
Murakami T (see Sudou et al). 2003;126:902-3 (Brief comm.)

Murashita T, Makino Y, Kamikubo Y, Yasuda K, Mabuchi M, Tamaki N. Quantitative gated myocardial perfusion single photon emission computed tomography improves the prediction of regional functional recovery in akinetic areas after coronary bypass surgery: useful tool for evaluation of myocardial viability. 2003;126:1328-34

Murata S (see Kown et al). 2003;126:1065-70

Murkin JM. Retrograde cerebral perfusion: more risk than benefit? 2003;126:631-3 (Editorial)

Murphy G (see Lim et al). 2003;126:1013-7

Murphy GJ, Nicholson ML. Rapamycin has no effect on fibrosisassociated gene expression or extracellular matrix accumulation when administered to animals with established or early allograft vasculopathy. 2003;126:2058-64

Murthy SC, Law S, Whooley BP, Alexandrou A, Chu K-M, Wong J. Atrial fibrillation after esophagectomy is a marker for postoperative morbidity and mortality. 2003;126:1162-7

Murthy SC (see DeCamp et al). 2003;126:17-27

Murthy SC (see Rice et al). 2003;126:1590-6

Mussa S, Guzik TJ, Black E, Dipp MA, Channon KM, Taggart DP. Comparative efficacies and durations of action of phenoxybenzamine, verapamil/nitroglycerin solution, and papaverine as topical antispasmodics for radial artery coronary bypass grafting. 2003;126:1798-805

Mussatto KA (see Ghanayem et al). 2003;126:1367-77

\section{$\mathrm{N}$}

Nabavi DG, Stockmann J, Schmid C, Schneider M, Hammel D, Scheld HH, Ringelstein EB. Doppler microembolic load predicts risk of thromboembolic complications in Novacor patients. 2003;126:160-7

Naeije R (see Wauthy et al). 2003;126:1434-41

Nafteux P (see Lerut et al). 2003;126:1121-8

Nagahiro I (see Date et al). 2003;126:476-81

Nagata N (see (Miyaji et al). 2003;126:1648-50 (Brief comm.)

Nagatsu M (see Isomatsu et al). 2003;126:1958-62 (Evolving tech.)

Nagele G (see Hangler et al). 2003;126:391-400

Naidu BV, Krishnadasan B, Farivar AS, Woolley SM, Thomas R, Van Rooijen N, Verrier ED, Mulligan MS. Early activation of the alveolar macrophage is critical to the development of lung ischemia-reperfusion injury. 2003;126:200-7

Naidu BV, Woolley SM, Farivar AS, Thomas R, Fraga C, Mulligan MS. Simvastatin ameliorates injury in an experimental model of lung ischemia-reperfusion. 2003;126:482-9

Naik M (see Taggart et al). 2003;126:1087-94

Nair S (see Stephen et al). 2003;126:2093-4 (Brief comm.)

Naito Y, Nakajima M, Inoue H, Mizutani E, Tsuchiya K. Successful treatment of a mycotic aortic arch aneurysm associated with an isolated left vertebral artery. 2003;126:883-5 (Brief comm.)

Naka Y (see Kherani et al). 2003;126:1634-5 (Brief comm.)

Naka Y (see Morgan et al). 2003;126:1188-90 (Brief comm.)

Naka Y (see Williams et al). 2003;126:436-41

Nakagawa K, Asamura H, Matsuno Y, Suzuki K, Kondo H, Maeshima A, Miyaoka E, Tsuchiya R. Thymoma: a clinico- pathologic study based on the new World Health Organization classification. 2003;126:1134-40

Nakagawa T, Minamiya Y, Katayose Y, Saito H, Taguchi K, Imano H, Watanabe H, Enomoto K, Sageshima M, Ueda T, Ogawa J-i. A novel method for sentinel lymph node mapping using magnetite in patients with non-small cell lung cancer. 2003;126:563-7

Nakajima M (see Naito et al). 2003;126:883-5 (Brief comm.)

Nakamura Y (see Ko et al). 2003;126:897-9 (Brief comm.)

Nakao S (see Uchiyama et al). 2003;126:148-59

Nakatani S (see Bando et al). 2003;126:358-64

Nakayama T (see Higashiyama et al). 2003;126:611 (Letter reply)

Nakazawa H (see Hayashi et al). 2003;126:1813-21

Nanton MA (see Baskett et al). 2003;126:232-9

Nardi C (see De Carlo et al). 2003;126:337-43

Nardi C (see Mecozzi et al). 2003;126:1198-9 (Brief comm.)

Naruke T (see Nomori et al). 2003;126:1584-9

Nascimbene S (see Melo et al). 2003;126:914-6 (Brief comm.)

Nasso G (see Gaudino et al). 2003;126:1107-12

Nasso G (see Gaudino et al). 2003;126:1968-71

Nataf P (see Attar et al). 2003;126:888-90 (Brief comm.)

Neligan S (see McMahon et al). 2003;126:1781-7

Netea MG (see Selzman et al). 2003;126:688-93

Neurologic Outcome Research Group (NORG) (see Scarborough et al). 2003;126:1561-7 (Evolving tech.)

Newberger JW (see Jonas et al). 2003;126:1765-74

Newburger JW (see Bellinger et al). 2003;126:1385-96

Newburger JW (see Wypij et al). 2003;126:1397-403

Newman MF (see Gaynor et al). 2003;126:1736-45

Newman MF (see Scarborough et al). 2003;126:1561-7 (Evolving tech.)

Nicholson ML (see Murphy and Nicholson). 2003;126:2058-64

Nicholson S (see Madan et al). 2003;126:1657-9 (Brief comm.)

Nicolini F (see Colli et al). 2003;126:881-3 (Brief comm.)

Nicolson SC (see Gaynor et al). 2003;126:1736-45

Nieuwland R (see van den Goor et al). 2003;126:1101-6

Niimi M (see Aramaki et al). 2003;126:853-4 (Brief comm.)

Nili N (see Fedak et al). 2003;126:797-806

Ninomiya H (see Uchiyama et al). 2003;126:148-59

Niranjan G (see Perreas et al). 2003;126:606-7 (Brief comm.)

Nishikawa M (see Kanemitsu et al). 2003;126:428-35

Nishimi M (see Teshima et al). 2003;126:401-7

Nishimura K (see Iwakura et al). 2003;126:1113-20

Nishimura K (see Koyama et al). 2003;126:2110-1 (Letter reply)

Nishimura M, Ogiwara M, Ishikawa M, Yatsu S, Masuoka A, Okamura N, Imanaka K, Kato M, Asano H, Kyo S. Fifteenmonth circulatory support for sustained ventricular fibrillation by left ventricular assist device. 2003;126:1190-2 (Brief comm.)

Nishimura M (see Gojo et al). 2003;126:885-6 (Brief comm.)

Nishio W (see Okada et al). 2003;126:558-62

Nishio W (see Okada et al). 2003;126:1911-5

Nitta T (see Ishii et al). 2003;126:254-62

Nitta Y, Tsuru Y, Yamaya K, Akasaka J, Oda K, Tabayashi K. Endovascular flexible stent grafting with arch vessel bypass for a case of aortic arch aneurysm. 2003;126:1186-8 (Brief comm.)

Niwaya K (see Bando et al). 2003;126:358-64

Noda H (see Takahashi et al). 2003;126:600-2 (Brief comm.) 
Nollert G, Miksch J, Kreuzer E, Reichart B. Risk factors for atherosclerosis and the degeneration of pericardial valves after aortic valve replacement. 2003;126:965-8

Nollert G, Oberhoffer M, Reichart B, Vicol C. Combination of the HEARTSTRING proximal seal system with a blower mister: a possible source of gas emboli. 2003;126:1192-4 (Brief comm.)

Nomori H, Ohtsuka T, Naruke T, Suemasu K. Histogram analysis of computed tomography numbers of clinical T1 N0 M0 lung adenocarcinoma, with special reference to lymph node metastasis and tumor invasiveness. 2003;126:1584-9

Nomura F (see Yoshitatsu et al). 2003;126:1531-6

Nomura K (see Ko et al). 2003;126:897-9 (Brief comm.)

Novak S (see Bolooki et al). 2003;126:374-85

Nussmeier N (see Koch et al). 2003;126:2032-43

Nussmeier N (see Koch et al). 2003;126:2044-51

Nussmeier NA. The female perspective: gender in cardiothoracic surgery. 2003;126:618-9 (Editorial)

\section{$\mathrm{O}$}

Obama T (see Watanabe et al). 2003;126:1212-4 (Brief comm.)

Oberbeck A (see Leyh et al). 2003;126:1000-4

Oberhoffer M (see Nollert et al). 2003;126:1192-4 (Brief comm.)

O'Brien MF (see Hogan and O'Brien). 2003;126:1251-3 (Editorial)

Ochoa ER (see Grikscheit et al). 2003;126:537-44

Oda K (see Nitta et al). 2003;126:1186-8 (Brief comm.)

Oda Y (see Yamaguchi et al). 2003;126:870-2 (Brief comm.)

Odim J (see Gupta et al). 2003;126:1746-52

Oechslin E (see Kaemmerer et al). 2003;126:1048-52

Oengoeren I (see Schmitz et al). 2003;126:1829-38

Ogawa J-i (see Nakagawa et al). 2003;126:563-7

Ogawa J-i (see Saito et al). 2003;126:609 (Letter reply)

Ogiwara M (see Imanaka et al). 2003;126:598-600 (Brief comm.)

Ogiwara M (see Nishimura et al). 2003;126:1190-2 (Brief comm.)

Oguzkaya F (see Bilgin et al). 2003;126:1580-3

O'Halloran E (see Gonzalez-Stawinski et al). 2003;126:1900-5

Ohashi H (see Yoshimura et al). 2003;126:1417-26

Ohno N, Fedak PWM, Weisel RD, Mickle DAG, Fujii T, Li R-K.

Transplantation of cryopreserved muscle cells in dilated cardiomyopathy: effects on left ventricular geometry and function. 2003;126:1537-48

Ohsawa H (see Watanabe et al). 2003;126:1212-4 (Brief comm.)

Ohta M, Sawabata N, Maeda H, Matsuda H. Safety of bronchoplastic resection after induction therapy for lung cancer. 2003; 126:1671 (Letter reply)

Ohta M (see Okumura et al). 2003;126:1922-8

Ohtsuka T (see Kitamura et al). 2003;126:904-5 (Brief comm.)

Ohtsuka T (see Nomori et al). 2003;126:1584-9

Ohtsuki S-i (see Sano et al). 2003;126:504-10

Oka S (see Ootaki et al). 2003;126:1411-6

Oka S (see Ootaki et al). 2003;126:1655-6 (Brief comm.)

Oka S (see Yoshimura et al). 2003;126:1417-26

Oka T (see Tagawa et al). 2003;126:1147-54

Okada M, Nishio W, Sakamoto T, Harada H, Uchino K, Tsubota $\mathrm{N}$. Long-term survival and prognostic factors of five-year survivors with complete resection of non-small cell carcinoma. $2003 ; 126: 558-62$
Okada M, Sakamoto T, Nishio W, Uchino K, Tsuboshima K, Tsubota N. Pleural lavage cytology in non-small cell lung cancer: lessons from 1000 consecutive resections. 2003;126: 1911-5

Okada T (see Uchiyama et al). 2003;126:148-59

Okamoto RJ, Xu H, Kouchoukos NT, Moon MR, Sundt TM III. The influence of mechanical properties on wall stress and distensibility of the dilated ascending aorta. 2003;126:842-50

Okamoto T (see Gotoh et al). 2003;126:1916-21

Okamura N (see Nishimura et al). 2003;126:1190-2 (Brief comm.)

Ökten İ (see Şahin et al). 2003;126:769-73

Okumura M, Ohta M, Takeuchi Y, Shiono H, Inoue M, Fukuhara K, Kadota Y, Miyoshi S, Fujii Y, Matsuda H. The immunologic role of thymectomy in the treatment of myasthenia gravis: implication of thymus-associated B-lymphocyte subset in reduction of the anti-acetylcholine receptor antibody titer. 2003; 126:1922-8

Oliaro A (see Filosso et al). 2003;126:1215-7 (Brief comm.)

Oliver JM, Garrido A, González A, Benito F, Mateos M, Aroca A, Sanz E. Rapid progression of midventricular obstruction in adults with double-chambered right ventricle. 2003;126:711-7

Oliveria SA (see Assad et al). 2003;126:300-2 (Brief comm.)

Olsen MA, Sundt TM, Lawton JS, Damiano RJ Jr, HopkinsBroyles D, Lock-Buckley P, Fraser VJ. Risk factors for leg harvest surgical site infections after coronary artery bypass graft surgery. 2003;126:992-9

Omar RZ, Morton LS, Murad S, Taylor KM. Use of flexibility tests in the manufacturing process of $60^{\circ}$ Björk-Shiley convexo-concave valves and the risk of outlet strut fracture. 2003; 126:832-6

Onishi K (see Yoshitatsu et al). 2003;126:1531-6

Ono M. Discussion of Fischer et al. 2003;126:1174-80

Onoda K (see Kanemitsu et al). 2003;126:428-35

Onorati F, Renzulli A, De Feo M, Santarpino G, Gregorio R, Biondi A, Cerasulo F, Cotrufo M. Does antegrade blood cardioplegia alone provide adequate myocardial protection in patients with left main stem disease? 2003;126:1345-51

Onorati F (see De Santo et al). 2003;126:826-31

Oo AY (see Conant et al). 2003;126:448-54

Ooi OC (see El Oakley et al). 2003;126:1668 (Letter)

Ootaki Y, Yamaguchi M, Yoshimura N, Oka S, Yoshida M, Hasegawa T. Secretion of A-type and B-type natriuretic peptides into the bloodstream and pericardial space in children with congenital heart disease. 2003;126:1411-6

Ootaki Y, Yamaguchi M, Yoshimura N, Oka S, Yoshida M, Hasegawa T. Unroofed coronary sinus syndrome: Diagnosis, classification, and surgical treatment. 2003;126:1655-6 (Brief comm.)

Oparil S. Improving outcomes for women after coronary artery bypass grafting: a case for prevention. 2003;126:1704-6 (Editorial)

Oron GH (see Walhout et al). 2003;126:521-8

Osawa H, Yoshii S, Hosaka S, Suzuki S, Abraham SJK, Tada Y. Left atrial dissection after aortic valve replacement. 2003;126: 604-5 (Brief comm.)

Oshima Y (see Yoshimura et al). 2003;126:1417-26

Oslund-Pinderski L (see Fischbein et al). 2003;126:216-23 
Osoegawa A (see Yamaguchi et al). 2003;126:870-2 (Brief comm.)

Ostermeyer J (see Oz et al). 2003;126:983-91

Otani H (see Uchiyama et al). 2003;126:148-59

Otsuji M (see Chiyo et al). 2003;126:1141-6

Ottavi P (see Puma et al). 2003;126:1998-2002

Ottenkamp J (see Schoof et al). 2003;126:282-3 (Brief comm.)

Oyer PE (see Demers et al). 2003;126:224-31

Oyer PE (see Lai et al). 2003;126:1978-86

Oz M (see Williams et al). 2003;126:436-41

Oz MC, Konertz WF, Kleber FX, Mohr FW, Gummert JF, Ostermeyer J, Lass M, Raman J, Acker MA, Smedira N. Global surgical experience with the Acorn cardiac support device. 2003;126:983-91

Oz MC (see Bisleri et al). 2003;126:1639-40 (Brief comm.)

Oz MC (see Kherani et al). 2003;126:1634-5 (Brief comm.)

Oz MC (see Morgan et al). 2003;126:582-3 (Brief comm.)

Oz MC (see Morgan et al). 2003;126:1188-90 (Brief comm.)

Oz MC (see Morgan et al). 2003;126:1624-33

\section{$\mathrm{P}$}

Pacini D, Mattioli S, Di Simone M, Ranocchi F, Grillone G, Di Bartolomeo R, Pierangeli A. Syphilitic aortic aneurysm: a rare case of tracheomalacia. 2003;126:900-2 (Brief comm.)

Paparella D (see Ibrahim et al). 2003;126:959-64

Papotti M (see Filosso et al). 2003;126:1215-7 (Brief comm.)

Pappone C (see Melo et al). 2003;126:914-6 (Brief comm.)

Parameshwar J (see Stoica et al). 2003;126:2072-4 (Brief comm.)

Paraskevaidis IA, Triantafilou K, Karatzas D, Kremastinos D Th. Right ventricular multiple myxomas obstructing right ventricular outflow tract. 2003;126:913-4 (Brief comm.)

Pardini A (see Puma et al). 2003;126:1998-2002

Parihar B (see Stephen et al). 2003;126:2093-4 (Brief comm.)

Park BJ, Flores R, Downey RJ, Bains MS, Rusch VW. Management of major hemorrhage during mediastinoscopy. 2003;126: 726-31

Park SJ. Discussion of Jonas et al. 2003;126:1765-74

Park YK (see Morgan et al). 2003;126:1188-90 (Brief comm.)

Parker JD (see Verma et al). 2003;126:1555-60 (Evolving tech.)

Parris T (see Samuels et al). 2003;126:2100-1 (Brief comm.)

Pasmantier MW (see Keresztes et al). 2003;126:1603-8

Pasquini L (see Formigari et al). 2003;126:1753-9

Pastorino U, Veronesi G, Landoni C, Leon M, Picchio M, Solli PG, Leo F, Spaggiari L, Pelosi G, Bellomi M, Fazio F. Fluorodeoxyglucose positron emission tomography improves preoperative staging of resectable lung metastasis. 2003;126:1906-10

Patachenko P (see Medalion et al). 2003;126:1875-9

Patel VS (see Petrofski et al). 2003;126:442-7

Patterson GA (see Force and Patterson). 2003;126:1247-50 (Clinical path. conf.)

Patterson GA (see Force et al). 2003;126:864-6 (Brief comm.)

Patterson GA (see Suda et al). 2003;126:1155-61

Patterson GA (see Tagawa et al). 2003;126:1147-54

Patterson GA. Discussion of Fischer et al. 2003;126:1174-80

Paxton LD (see Jones et al). 2003;126:303 (Letter)
Pearl JM. Right ventricular-pulmonary artery connection in stage 1 palliation of hypoplastic left heart syndrome. 2003;126:1268-70 (Editorial)

Pearl JM (see Border et al). 2003;126:1760-4

Pehkonen E (see Wu et al). 2003;126:1477-82

Pelosi G (see Pastorino et al). 2003;126:1906-10

Peniston CM (see Peterson et al). 2003;126:1314-9

Perreas KG, Niranjan G, Clark S. Automated proximal anastomosis for redo coronary artery bypass grafting through a lateral thoracotomy. 2003;126:606-7 (Brief comm.)

Perry SB (see Brown et al). 2003;126:272-81

Péterffy Á (see Galajda et al). 2003;126:2107 (Letter reply)

Petersen G, Martin U, Singhal A, Criner GJ. Intralobar sequestration in the middle-aged and elderly adult: recognition and radiographic evaluation. 2003;126:2086-90 (Brief comm.)

Peterson MD, Borger MA, Rao V, Peniston CM, Feindel CM. Skeletonization of bilateral internal thoracic artery grafts lowers the risk of sternal infection in patients with diabetes. 2003; 126:1314-9

Petro KR (see Lawton et al). 2003;126:936-8 (Editorial)

Petrofski JA, Patel VS, Russell SD, Milano CA. BVS 5000 support after cardiac transplantation. 2003;126:442-7

Petrucci RJ. Finding our way from the heart to the head. 2003; 126:944-6 (Editorial)

Pett SB (see Dietl et al). 2003;126:1181-3 (Brief comm.)

Pettenazzo E (see Bottio et al). 2003;126:99-105

Pettersson G (see Gillinov et al). 2003;126:1661-2 (Letter)

Pettersson GB (see Lam et al). 2003;126:2101-3 (Brief comm.)

Pfammatter J-P (see Gessler et al). 2003;126:718-25

Pfannschmidt J, Muley T, Hoffmann H, Dienemann H. Prognostic factors and survival after complete resection of pulmonary metastases from colorectal carcinoma: experiences in 167 patients. 2003;126:732-9

Pfenninger J (see Gessler et al). 2003;126:718-25

Picca S (see Carotti et al). 2003;126:1839-50

Picchio FM (see Formigari et al). 2003;126:1753-9

Picchio M (see Pastorino et al). 2003;126:1906-10

Pierangeli A (see Pacini et al). 2003;126:900-2 (Brief comm.)

Pigula FA, Mahnke B, Agnastopolous P, Casta A, Munoz R, Gandhi SK. Closed correction of systemic semilunar valve insufficiency in the neonate. 2003;126:1650-2 (Brief comm.)

Pigula FA. Discussion of Ghanayem et al. 2003;126:1367-77

Pilla JJ, Blom AS, Brockman DJ, Ferrari VA, Yaun Q, Acker MA. Passive ventricular constraint to improve left ventricular function and mechanics in an ovine model of heart failure secondary to acute myocardial infarction. 2003;126:1467-76

Pintucci G (see Bizekis et al). 2003;126:659-665. Correction 2003; 126:1680

Pistecky PV (see Scheltes et al). 2003;126:191-9 (Evolving tech.)

Pittarello G (see Bottio et al). 2003;126:1194-5 (Brief comm.)

Pizarro C. Discussion of Fedak et al. 2003;126:797-806

Pizarro C. Discussion of Sano et al. 2003;126:504-10

Plante J (see Lacasse et al). 2003;126:761-8

Plunkett M (see Marelli et al). 2003;126:821-5

Pohl C (see Schmitz et al). 2003;126:1829-38

Pokrovsky SN, Ezhov MV, Il'ina LN, Afanasieva OI, Sinitsyn VY, Shiriaev AA, Akchurin RS. Association of lipoprotein(a) 
excess with early vein graft occlusions in middle-aged men undergoing coronary artery bypass surgery. 2003;126:1071-5 Pola P (see Gaudino et al). 2003;126:1968-71

Popilskis S (see He et al). 2003;126:56-65

Popoff G (see Bonacchi et al). 2003;126:1302-13

Porat EE (see Estrera et al). 2003;126:1288-95

Porcaro G (see Puma et al). 2003;126:1998-2002

Port JL (see Keresztes et al). 2003;126:1603-8

Possati G (see Gaudino et al). 2003;126:1107-12

Possati G (see Gaudino et al). 2003;126:1968-71

Posther KE (see Reed et al). 2003;126:1943-51

Postmus PE (see van der Maten et al). 2003;126:740-3

Power JM (see Raman et al). 2003;126:1357-66

Pozzi M. Discussion of Sano et al. 2003;126:504-10

Prasad S (see Damiano et al). 2003;126:2016-21

Prasad SM, Maniar HS, Camillo CJ, Schuessler RB, Boineau JP, Sundt TM III, Cox JL, Damiano RJ Jr. The Cox maze III procedure for atrial fibrillation: long-term efficacy in patients undergoing lone versus concomitant procedures. 2003;126:1822-8

Pratali S (see Mecozzi et al). 2003;126:1198-9 (Brief comm.)

Prefumo F (see Jahangiri et al). 2003;126:894-895 (Brief comm.) Correction 2003;126:1680

Preusse CJ (see Schmitz et al). 2003;126:645-50

Prieto LR (see Duncan et al). 2003;126:694-702

Prifti E (see Bonacchi et al). 2003;126:1302-13

Prins TR (see Drenth et al). 2003;126:1184-5 (Brief comm.)

Pritisanac A (see Gulbins et al). 2003;126:890-1 (Brief comm.)

Profaizer T (see Hawkins et al). 2003;126:247-53

Puma F, Fedeli C, Ottavi P, Porcaro G, Fonsi GB, Pardini A, Daddi G. Laparoscopic omental flap for the treatment of major sternal wound infection after cardiac surgery. 2003;126:19982002

Pumphrey C (see Jahangiri et al). 2003;126:894-895 (Brief comm.) Correction 2003;126:1680

Puskas JD (see Corvera et al). 2003;126:1549-54

Puttagunta L (see McNamee et al). 2003;126:861-3 (Brief comm.)

Pyeritz RE. Express yourself-but consider the consequences. 2003;126:334-6 (Editorial)

\section{Q}

Qiao J-H (see Marchevsky et al). 2003;126:551-7

Quader MA. Discussion of Ikonomidis et al. 2003;126:2022-31

Quadri SM. Discussion of Yarbrough et al. 2003;126:1892-9

Quardt SM (see Wells et al). 2003;126:498-503

Quarti A (see Alfieri et al). 2003;126:75-9

Qureshi A (see Duncan et al). 2003;126:694-702

\section{$\mathrm{R}$}

Raby B (see Lacasse et al). 2003;126:761-8

Raczkowski AR (see Wolf et al). 2003;126:168-78

Radley-Smith R (see McMahon et al). 2003;126:1781-7

Räisänen T (see Kakko et al). 2003;126:106-13

Raiter A (see Rzyman et al). 2003;126:755-60

Raji MR (see Tossios et al). 2003;126:1513-20
Rakowski H (see David et al). 2003;126:2118-9 (Letter reply)

Raman J, Ishikawa S, Storer MM, Power JM. Surgical radiofrequency ablation of both atria for atrial fibrillation: results of a multicenter trial. 2003;126:1357-66

Raman J (see Oz et al). 2003;126:983-91

Raman JS (see Shah et al). 2003;126:1320-7

Raman JS (see Shah et al). 2003;126:1972-7

Rangappa S, Entwistle JWC, Wechsler AS, Kresh JY. Cardiomyocyte-mediated contact programs human mesenchymal stem cells to express cardiogenic phenotype. 2003;126:124-32

Ranocchi F (see Pacini et al). 2003;126:900-2 (Brief comm.)

Rao JN (see Clark et al). 2003;126:2052-7

Rao V (see Peterson et al). 2003;126:1314-9

Rao V. Discussion of Blackstone et al. 2003;126:783-96

Rappaport LA (see Bellinger et al). 2003;126:1385-96

Rappaport LA (see Wypij et al). 2003;126:1397-403

Rappaport LJ (see Jonas et al). 2003;126:1765-74

Rastan A (see Falk et al). 2003;126:1575-9 (Evolving tech.)

Reardon MJ. Discussion of Absi et al. 2003;126:344-57

Rebe P (see Leyh et al). 2003;126:1000-4

Rebello K (see Bonanomi et al). 2003;126:851-2 (Evolving tech.)

Reddy M. Discussion of Carotti et al. 2003;126:1839-50

Redmann K (see Lunkenheimer et al). 2003;126:592-6 (Brief comm.)

Redonnet M (see Litzler et al). 2003;126:1204-7 (Brief comm.)

Reed CE, Harpole DH, Posther KE, Woolson SL, Downey RJ, Meyers BF, Heelan RT, MacApinlac HA, Jung S-H, Silvestri GA, Siegel BA, Rusch VW. Results of the American College of Surgeons Oncology Group Z0050 Trial: the utility of positron emission tomography in staging potentially operable non-small cell lung cancer. 2003;126:1943-51

Rees P (see McMahon et al). 2003;126:1781-7

Reeves B (see Caputo et al). 2003;126:39-47

Reichart B (see Gulbins et al). 2003;126:890-1 (Brief comm.)

Reichart B (see Nollert et al). 2003;126:965-8

Reichart B (see Nollert et al). 2003;126:1192-4 (Brief comm.)

Reisli I, Aribas OK, Koksal Y, Avunduk MC, Koc H. A giant gastroenteric cyst associated with pectus excavatum and compression of the thoracic duct: a case report. 2003;126:584-5 (Brief comm.)

Reitz BA (see Demers et al). 2003;126:224-31

Reitz BA (see Lai et al). 2003;126:1978-86

Renzulli A (see De Santo et al). 2003;126:826-31

Renzulli A (see Onorati et al). 2003;126:1345-51

Rescigno G, Matteucci MLS, Iacovoni A, Banfi C, Seddio F, Lorini L, Giamundo B, Ferrazzi P. Systolic anterior motion after mitral valve repair: myectomy as an alternative solution. 2003;126:1196-7 (Brief comm.)

Reznikov LL (see Selzman et al). 2003;126:688-93

Rhodes JF (see Duncan et al). 2003;126:694-702

Rhodes L (see Madan et al). 2003;126:1657-9 (Brief comm.)

Rice T. Discussion of Roberts et al. 2003;126:1597-602

Rice TW, Adelstein DJ, Chidel MA, Rybicki LA, DeCamp MM, Murthy SC, Blackstone EH. Benefit of postoperative adjuvant chemoradiotherapy in locoregionally advanced esophageal carcinoma. 2003;126:1590-6

Rice TW (see DeCamp et al). 2003;126:17-27

Rice TW (see Gillinov et al). 2003;126:1661-2 (Letter) 
Richenbacher W. Cardiac transplantation in prisoners. 2003;126: 1226 (Letter)

Richter M (see Go et al). 2003;126:867-9 (Brief comm.)

Riddoch MJ (see Harrington et al). 2003;126:638-44

Riedel M. Indications for pulmonary endarterectomy. 2003;126: 1227-8 (Letter)

Ring WS. Discussion of Hawkins et al. 2003;126:247-53

Ringelstein EB (see Nabavi et al). 2003;126:160-7

Riquet M (see Barthes et al). 2003;126:1199-200 (Brief comm.)

Ritter J. Discussion of Force and Patterson. 2003;126:1247-50 (Clinical path. conf.)

Riviere C (see Bonanomi et al). 2003;126:851-2 (Evolving tech.)

Rizzoli G, Bottio T, Thiene G, Toscano G, Casarotto D. Long-term durability of the Hancock II porcine bioprosthesis. 2003;126: 66-74

Rizzoli G (see Blackstone et al). 2003;126:783-96

Rizzoli G (see Bottio et al). 2003;126:99-105

Rizzoli G (see Bottio et al). 2003;126:1352-6

Robbins RC (see Demers et al). 2003;126:224-31

Robbins RC (see Kofidis et al). 2003;126:2113-4 (Letter)

Robbins RC (see Kown et al). 2003;126:1065-70

Robbins RC (see Lai et al). 2003;126:1978-86

Roberts PF, Straznicka M, Lara PN, Lau DH, Follette DM, Gandara DR, Benfield JR. Resection of multifocal non-small cell lung cancer when the bronchioloalveolar subtype is involved. 2003; 126:1597-602

Robicsek F. "Cinching" the mitral valve. 2003;126:942-3 (Editorial)

Robicsek F. Priority issue: who is on first base? 2003;126:321-2 (Editorial)

Robicsek F. Robotic cardiac surgery: Quo vadis? 2003;126:623-4 (Editorial)

Rodriquez LL (see Gillinov et al). 2003;126:313-6 (Editorial)

Roemer G (see Fratz et al). 2003;126:1724-9

Romano G (see De Santo et al). 2003;126:826-31

Romero M (see Klena et al). 2003;126:857-9 (Brief comm.)

Roper C. Discussion of Force and Patterson. 2003;126:1247-50 (Clinical path. conf.)

Rosalion A (see Shah et al). 2003;126:1320-7

Rosalion A (see Shah et al). 2003;126:1972-7

Rosengart T. Discussion of Saito et al. 2003;126:114-23

Rosenthal GL (see Duncan et al). 2003;126:694-702

Rosenthal GL (see Lupinetti et al). 2003;126:240-6

Ross D (see Ardehali et al). 2003;126:1929-34

Ross DB (see Baskett et al). 2003;126:232-9

Ross JD (see Jonjev et al). 2003;126:1880-5

Roth M (see Schoenburg et al). 2003;126:1455-60

Roth SJ (see Jonas et al). 2003;126:1765-74

Rothbard J B (see Kown et al). 2003;126:1065-70

Roussel JC (see Mathieu et al). 2003;126:1449-54

Rudd NA (see Ghanayem et al). 2003;126:1367-77

Ruel M, Bianchi C, Khan TA, Xu S, Liddicoat JR, Voisine P, Araujo E, Lyon H, Kohane IS, Libermann TA, Sellke FW. Gene expression profile after cardiopulmonary bypass and cardioplegic arrest. 2003;126:1521-30

Ruffini E (see Dato et al). 2003;126:408-14

Ruffini E (see Filosso et al). 2003;126:1215-7 (Brief comm.)

Ruohtula T (see Sihvo et al). 2003;126:1952-7
Rusch VW (see Flores et al). 2003;126:11-6

Rusch VW (see Park et al). 2003;126:726-31

Rusch VW (see Reed et al). 2003;126:1943-51

Russell H (see Ardehali et al). 2003;126:1929-34

Russell SD (see Petrofski et al). 2003;126:442-7

Rutten PM (see van den Goor et al). 2003;126:1101-6

Ruttmann E (see Hangler et al). 2003;126:391-400

Rybicki LA (see DeCamp et al). 2003;126:17-27

Rybicki LA (see Rice et al). 2003;126:1590-6

Rzyman W, Dziadziuszko R, Skokowski J, Wilimski R, Raiter A, Szymanowska A, Jassem J. The influence of blood transfusion on survival in operated non-small cell lung cancer patients. 2003; $126: 755-60$

\section{S}

Sablayrolles J-L (see Attar et al). 2003;126:888-90 (Brief comm.) Sade RM. Cardiac transplantation in prisoners. 2003;126:1227 (Letter reply)

Sade RM (see Ikonomidis et al). 2003;126:2022-31

Safi HJ (see Estrera et al). 2003;126:1288-95

Sageshima M (see Nakagawa et al). 2003;126:563-7

Şahin E, Enön S, Cangır AK, Kutlay H, Kavukçu Ş, Akay H, Ökten İ, Yavuzer Ş. Single-stage transthoracic approach for right lung and liver hydatid disease. 2003;126:769-73

Saito H, Minamiya Y, Ogawa J-i. Preoperative localization techniques during thoracoscopic operations. 2003;126:609 (Letter reply)

Saito H (see Nakagawa et al). 2003;126:563-7

Saito T, Kuang J-Q, Lin CCH, Chiu RC-J. Transcoronary implantation of bone marrow stromal cells ameliorates cardiac function after myocardial infarction. 2003;126:114-23

Saitoh Y (see Chiyo et al). 2003;126:1141-6

Sakai S (see Gavard et al). 2003;126:807-13

Sakamoto S-i (see Ishii et al). 2003;126:254-62

Sakamoto T (see Okada et al). 2003;126:558-62

Sakamoto T (see Okada et al). 2003;126:1911-5

Sakano H (see Sugi et al). 2003;126:568-73

Sakiyama S, Kondo K, Matsuoka H, Yoshida M, Miyoshi T, Yoshida S, Monden Y. Fatal air embolism during computed tomography-guided pulmonary marking with a hook-type marker. 2003;126:1207-9 (Brief comm.)

Sakopoulos AG (see van Dongen et al). 2003;126:703-10

Sakurai M (see Takahashi et al). 2003;126:1461-6

Saleh S (see Castellá et al). 2003;126:1442-8

Salmon AP (see Monro et al). 2003;126:511-20

Salo JA (see Sihvo et al). 2003;126:1952-7

Sames E (see Mair et al). 2003;126:1378-84

Sample JA (see Yarbrough et al). 2003;126:1489-97

Sample JA (see Yarbrough et al). 2003;126:1892-9

Samuels LE, Entwistle JC III, Holmes EC, Parris T, Wechsler AS. Mechanical support of the unrepaired postinfarction ventricular septal defect with the Abiomed BVS 5000 ventricular assist device. 2003;126:2100-1 (Brief comm.)

Sandner D (see Czerny et al). 2003;126:916-8 (Brief comm.)

Sandor A (see Conlan and Sandor). 2003;126:2083-5 (Brief comm.)

Sani G (see Bonacchi et al). 2003;126:1302-13 
Sano S, Ishino K, Kawada M, Arai S, Kasahara S, Asai T, Masuda Z-i, Takeuchi M, Ohtsuki S-i. Right ventricle-pulmonary artery shunt in first-stage palliation of hypoplastic left heart syndrome. 2003;126:504-10

Sano S (see Ishino and Sano). 2003;126:1653-4 (Brief comm.)

Sano Y (see Date et al). 2003;126:476-81

Santamaria P (see Abad and Santamaria). 2003;126:1229 (Letter)

Santamore WP (see He et al). 2003;126:56-65

Santarpino G (see Onorati et al). 2003;126:1345-51

Santinelli V (see Melo et al). 2003;126:914-6 (Brief comm.)

Santoliquido A (see Gaudino et al). 2003;126:1968-71

Santos A (see Assad et al). 2003;126:300-2 (Brief comm.)

Sanz E (see Oliver et al). 2003;126:711-7

Saponara F (see Bizekis et al). 2003;126:659-665. Correction 2003; $126: 1680$

Sarkar K (see Vannan and Sarkar). 2003;126:317-20 (Editorial)

Sasaki N (see Senzaki et al). 2003;126:1645-7 (Brief comm.)

Sasson L (see Medalion et al). 2003;126:1875-9

Sato H (see Gojo et al). 2003;126:885-6 (Brief comm.)

Sato M (see Sugawara et al). 2003;126:1225 (Letter reply)

Satoh T (see Bando et al). 2003;126:358-64

Satsu T (see Yamada et al). 2003;126:597-8 (Brief comm.)

Saunders AM (see Gaynor et al). 2003;126:1736-45

Savage EB. Study of warm perfusion rather than cardioplegia. 2003;126:2111 (Letter)

Savolainen MJ (see Kakko et al). 2003;126:106-13

Sawa Y (see Hayashi et al). 2003;126:1813-21

Sawabata N. Malignant status at surgical margin of limited-resected non-small cell lung cancer: a crucial finding for predicting local relapse. 2003;126:610-1 (Letter)

Sawabata N (see Ohta et al). 2003;126:1671 (Letter reply)

Saxena R, Lemmon J, Ellis J, Yoganathan A. An in vitro assessment by means of laser Doppler velocimetry of the Medtronic Advantage bileaflet mechanical heart valve hinge flow. 2003; 126:90-8

Scarborough JE, White W, Derilus FE, Mathew JP, Newman MF, Landolfo KP, The Neurologic Outcome Research Group (NORG). Combined use of off-pump techniques and a sutureless proximal aortic anastomotic device reduces cerebral microemboli generation during coronary artery bypass grafting. 2003;126:1561-7 (Evolving tech.)

Schachner A (see Medalion et al). 2003;126:1875-9

Schäfers H-J (see Langer et al). 2003;126:874-5 (Brief comm.)

Schaff HV. Discussion of Prasad et al. 2003;126:1822-8

Schaper M (see Frese et al). 2003;126:748-54

Scheld HH (see Lunkenheimer et al). 2003;126:592-6 (Brief comm.)

Scheld HH (see Nabavi et al). 2003;126:160-7

Scheltes JS, van Andel CJ, Pistecky PV, Borst C. Coronary anastomotic devices: blood-exposed non-intimal surface and coronary wall stress. 2003;126:191-9 (Evolving tech.)

Schiavello R (see Gaudino et al). 2003;126:1107-12

Schiller W (see Schmitz et al). 2003;126:645-50

Schmid C (see Nabavi et al). 2003;126:160-7

Schmid RA (see Frese et al). 2003;126:748-54

Schmidt D (see Albes et al). 2003;126:1504-12

Schmitz C, Ashraf O, Schiller W, Preusse CJ, Esmailzadeh B, Likungu JA, Fimmers R, Welz A. Transit time flow measure- ment in on-pump and off-pump coronary artery surgery. 2003; 126:645-50

Schmitz C, Weinreich S, White J, Oengoeren I, Schneider R, Schneider D, Speth I, Pohl C, Welz A. Can particulate extraction from the ascending aorta reduce neurologic injury in cardiac surgery? 2003;126:1829-38

Schneider D (see Schmitz et al). 2003;126:1829-38

Schneider M (see Nabavi et al). 2003;126:160-7

Schneider R (see Schmitz et al). 2003;126:1829-38

Schoenburg M, Kraus B, Muehling A, Taborski U, Hofmann H, Erhardt G, Hein S, Roth M, Vogt PR, Karliczek GF, Kloevekorn WP. The dynamic air bubble trap reduces cerebral microembolism during cardiopulmonary bypass. 2003;126:1455-60

Schoof PH, Tjien ATJ, Lam J, Kuipers I, Ottenkamp J, Hazekamp M, Dion R. Valve-sparing aortic root reconstruction with a valveless aortic allograft. 2003;126:282-3 (Brief comm.)

Schoof PH (see Lalezari et al). 2003;126:1053-60

Schoof PH (see Leeuwenburgh et al). 2003;126:2105-6 (Letter reply)

Schramm R (see Langer et al). 2003;126:874-5 (Brief comm.)

Schuessler RB (see Prasad et al). 2003;126:1822-8

Schuessler RP (see Damiano et al). 2003;126:2016-21

Schwann N (see Koch et al). 2003;126:932-5 (Editorial)

Schwann NM, Chaney MA. No pain, much gain? 2003;126:1261-4 (Editorial)

Schwarzman D (see Bonanomi et al). 2003;126:1859-66

Schwertz DW (see Jonjev et al). 2003;126:1880-5

Sciarra M (see Carotti et al). 2003;126:1839-50

Sebening W (see Fratz et al). 2003;126:1724-9

Seddio F (see Rescigno et al). 2003;126:1196-7 (Brief comm.)

Seevanayagam S (see Shah et al). 2003;126:1972-7

Sehic A (see Yeh et al). 2003;126:589-91 (Brief comm.)

Sekarski N (see Corno et al). 2003;126:1775-80

Sekine Y (see Chiyo et al). 2003;126:1141-6

Sellke FW. The peer-review process in medical publishing: a reviewer's perspective. 2003;126:1683-5 (Editorial)

Sellke FW (see Ruel et al). 2003;126:1521-30

Sellke FW. Discussion of Absi et al. 2003;126:344-57

Sellke FW. Discussion of Fedak et al. 2003;126:797-806

Sellke FW. Discussion of Fischer et al. 2003;126:1174-80

Sellke FW. Discussion of Szabó et al. 2003;126:651-8

Selzman CH, Netea MG, Zimmerman MA, Weinberg A, Reznikov LL, Grover FL, Dinarello CA. Atherogenic effects of Chlamydia pneumoniae: refuting the innocent bystander hypothesis. 2003;126:688-93

Sengul AT (see Basoglu et al). 2003;126:1201-2 (Brief comm.)

Senzaki H, Asano H, Masutani S, Matunaga T, Ishido H, Taketatu M, Kobayashi T, Sasaki N, Kyo S, Yokote Y. Anomalous origin of the left coronary artery from the main pulmonary artery associated with Berry syndrome. 2003;126:1645-7 (Brief comm.)

Sergeant P, Meyns B, Wouters P, Demeyere R, Lauwers P. Longterm outcome after coronary artery bypass grafting in cardiogenic shock or cardiopulmonary resuscitation. 2003;126:1279-87

Serricchio M (see Gaudino et al). 2003;126:1968-71 
Setser RM, Kasper JM, Lieber ML, Starling RC, McCarthy PM, White RD. Persistent abnormal left ventricular systolic torsion in dilated cardiomyopathy after partial left ventriculectomy. 2003;126:48-55

Seu P (see DiBardino et al). 2003;126:859-61 (Brief comm.)

Shackcloth MJ (see Conant et al). 2003;126:448-54

Shaddy RE (see Hawkins et al). 2003;126:247-53

Shah PJ, Gordon I, Fuller J, Seevanayagam S, Rosalion A, Tatoulis J, Raman JS. Factors affecting saphenous vein graft patency: clinical and angiographic study in 1402 symptomatic patients operated on between 1977 and 1999. 2003;126:1972-7

Shah PJ, Hare DL, Raman JS, Gordon I, Chan RK, Horowitz JD, Rosalion A, Buxton BF. Survival after myocardial revascularization for ischemic cardiomyopathy: a prospective ten-year follow-up study. 2003;126:1320-7

Sharony R (see Bizekis et al). 2003;126:659-665 (Brief comm.) Correction 2003;126:1680

Sharony R (see Grossi et al). 2003;126:922 (Letter)

Sheinbaum R (see Estrera et al). 2003;126:1288-95

Shemin R. Discussion of Schmitz et al. 2003;126:1829-38

Shen M (see Zegdi et al). 2003;126:1867-74

Shibata K (see Kitamura et al). 2003;126:904-5 (Brief comm.)

Shibuya K (see Chiyo et al). 2003;126:1141-6

Shiku H (see Kanemitsu et al). 2003;126:428-35

Shimabukuro K (see Mori et al). 2003;126:415-9

Shimazu M (see Aramaki et al). 2003;126:853-4 (Brief comm.)

Shimizu J (see He et al). 2003;126:56-65

Shimizu N (see Date et al). 2003;126:476-81

Shimono T (see Kanemitsu et al). 2003;126:428-35

Shimpo H (see Kanemitsu et al). 2003;126:428-35

Shingu K (see Uchiyama et al). 2003;126:148-59

Shinkawa T (see Yamagishi et al). 2003;126:1218-9 (Brief comm.)

Shin'oka T (see Isomatsu et al). 2003;126:1958-62 (Evolving tech.)

Shiono H (see Okumura et al). 2003;126:1922-8

Shirasugi N (see Aramaki et al). 2003;126:853-4 (Brief comm.)

Shiriaev AA (see Pokrovsky et al). 2003;126:1071-5

Shirota T (see He et al). 2003;126:455-64

Shpiner R (see Ardehali et al). 2003;126:1929-34

Shuhaiber JH. Asymptomatic mitral valve incompetence: is there evidence for surgery? 2003;126:2117-8 (Letter)

Shuhaiber JH. Massad M, Geha A. Study design in valve surgery and outcome. 2003;126:1660-1 (Letter)

Shukla V (see Stephen et al). 2003;126:2093-4 (Brief comm.)

Shumway NE (see Lai et al). 2003;126:1978-86

Shuntoh K (see Yamagishi et al). 2003;126:1218-9 (Brief comm.)

Sidi D (see Boudjemline et al). 2003;126:490-7

Sidi D (see Corno et al). 2003;126:1775-80

Siegel B. Discussion of Force and Patterson. 2003;126:1247-50 (Clinical path. conf.)

Siegel BA (see Reed et al). 2003;126:1943-51

Siegemund A (see Albes et al). 2003;126:1504-12

Sievers EM (see DiPerna et al). 2003;126:1129-33

Sievers HH. Chronic and adjustable pulmonary artery banding: reflections on old knowledge. 2003;126:2104-5 (Letter)

Sihvo EIT, Ruohtula T, Auvinen MI, Kiovistoinen A, Harjula AL, Salo JA. Simultaneous progression of oxidative stress and angiogenesis in malignant transformation of Barrett esophagus. 2003;126:1952-7

Silkoff PE (see Adatia et al). 2003;126:1674-5 (Letter reply)

Silvestri GA (see Reed et al). 2003;126:1943-51

Simchen E (see Galai et al). 2003;126:1018-25

Simon AR (see Fischer et al). 2003;126:1641-3 (Brief comm.)

Simpson AWM (see Conant et al). 2003;126:448-54

Simsic JM, Bradley SM, Mulvihill DM. Sodium nitroprusside infusion after bidirectional superior cavopulmonary connection: preserved cerebral blood flow velocity and systemic oxygenation. 2003;126:186-90

Simsic JM (see Bradley et al). 2003;126:1033-9

Singhal A (see Petersen et al). 2003;126:2086-90 (Brief comm.)

Sinitsyn VY (see Pokrovsky et al). 2003;126:1071-5

Sirlak M, Eryilmaz S, Yazicioglu L, Kiziltepe U, Eyileten Z, Durdu MS, Tasoz R, Eren NT, Aral A, Kaya B, Akalin H. Comparative study of microfibrillar collagen hemostat (Colgel) and oxidized cellulose (Surgicel) in high transfusion-risk cardiac surgery. 2003;126:666-70

Sivaprakasam R (see Zamvar and Sivaprakasam). 2003;126:923 (Letter)

Skema C (see Friedberg et al). 2003;126:1963-7 (Evolving tech.) Skokowski J (see Rzyman et al). 2003;126:755-60

Smedira N (see Oz et al). 2003;126:983-91

Smith AL (see Trachiotis et al). 2003;126:2065-71

Smith CR Jr (see Morgan et al). 2003;126:582-3 (Brief comm.)

Smith ML (see Domkowski et al). 2003;126:386-90

Smythe WR. Discussion of Flores et al. 2003;126:11-6

Soga Y (see Koyama et al). 2003;126:2110-1 (Letter reply)

Solli PG (see Pastorino et al). 2003;126:1906-10

Song DH, Agarwal JP, Jeevanandam V. Rigid sternal fixation in the cardiac transplant population. 2003;126:896-7 (Brief comm.)

Song M. Discussion of Schmitz et al. 2003;126:1829-38

Sonmez B, Demirsoy E, Yilmaz O. Atrioesophageal fistula: Is it an unavoidable complication of radiofrequency ablation? 2003; 126:1662-3 (Letter)

Soós P (see Kollár et al). 2003;126:977-82

Sortini A, Sortini D, Carrella G. Negative aspects of preoperative delay in early stage non-small cell lung cancer. 2003;126: 609-10 (Letter)

Sortini A, Sortini D, Carrella G. Preoperative localization techniques during thoracoscopic operations. 2003;126:608-9 (Letter)

Sortini D (see Sortini et al). 2003;126:608-9 (Letter)

Sortini D (see Sortini et al). 2003;126:609-10 (Letter)

Souilamas R (see Barthes et al). 2003;126:1199-200 (Brief comm.)

Spaggiari L (see Pastorino et al). 2003;126:1906-10

Spaggiari L (see Veronesi et al). 2003;126:1670-1 (Letter)

Spatuzza P (see Gaudino et al). 2003;126:1968-71

Speth I (see Schmitz et al). 2003;126:1829-38

Spielvogel D (see Hagl et al). 2003;126:1005-12

Spies C (see von Heymann et al). 2003;126:1667-8 (Letter)

Spinale FG (see Yarbrough et al). 2003;126:1489-97

Spinale FG (see Yarbrough et al). 2003;126:1892-9

Spray TL (see Gaynor et al). 2003;126:1736-45

Spray TL. Discussion of Ghanayem et al. 2003;126:1367-77

Squitieri R (see Hagl et al). 2003;126:1005-12 
Srinivasan A (see Grikscheit et al). 2003;126:537-44

Stahl KD (see Boyd and Stahl). 2003;126:625-30 (Editorial)

Stamatis G (see Marra and Stamatis). 2003;126:1665-6 (Letter reply)

Stamm C (see Friehs et al). 2003;126:263-71

Stanton JJ (see Lunkenheimer et al). 2003;126:592-6 (Brief comm.)

Starling RC (see Setser et al). 2003;126:48-55

Starnes VA (see DiPerna et al). 2003;126:1129-33

Starnes VA (see Haddy and Starnes). 2003;126:2106 (Letter)

Starnes VA. Discussion of Jonas et al. 2003;126:1765-74

Starnes VA. Discussion of Wells et al. 2003;126:498-503

Starr JP (see Bacha et al). 2003;126:1718-23

Stayer SA (see Andropoulos et al). 2003;126:1712-7

Steendijk P (see Leeuwenburgh et al). 2003;126:2105-6 (Letter reply)

Stein H (see Falk et al). 2003;126:1575-9 (Evolving tech.)

Steiner J (see Mair et al). 2003;126:1378-84

Steltzer MM (see Ghanayem et al). 2003;126:1367-77

Stephen T, Thankachen R, Parihar B, Nair S, Shukla V. Multilocular tuberculous cyst of thymus gland. 2003;126:2093-4 (Brief comm.)

Stewart RH (see Fischer et al). 2003;126:1483-8

Stiko A (see Yarbrough et al). 2003;126:1892-9

Stocke K (see Gavard et al). 2003;126:807-13

Stockmann J (see Nabavi et al). 2003;126:160-7

Stöhr IM (see Albes et al). 2003;126:1504-12

Stoica SC, Goddard M, Tsui S, Dunning J, McNeil K, Parameshwar J, Large SR. Ventricular assist surprise: giant cell myocarditis or sarcoidosis? 2003;126:2072-4 (Brief comm.)

Storer MM (see Raman et al). 2003;126:1357-66

Strauss BH (see Fedak et al). 2003;126:797-806

Straznicka M (see Roberts et al). 2003;126:1597-602

Stroud MR (see Ikonomidis et al). 2003;126:2022-31

Stroud RE (see Yarbrough et al). 2003;126:1489-97

Strüber M (see Fischer et al). 2003;126:1641-3 (Brief comm.)

Stumpf N (see Szabó et al). 2003;126:651-8

Sturk A (see van den Goor et al). 2003;126:1101-6

Su W-J (see Hwang et al). 2003;126:1643-4 (Brief comm.)

Suda T (see Tagawa et al). 2003;126:1147-54

Suda T, Tagawa T, Kanaan SA, Kozower BD, Daddi N, Mohanakumar T, Patterson GA. Adenovirus encoding soluble tumor necrosis factor $\alpha$ receptor immunoglobulin prolongs gene expression of a cotransfected reporter gene in rat lung. 2003;126: 1155-61

Sudoh M (see Sugi et al). 2003;126:568-73

Sudou M, Sugi K, Murakami T. Bronchioloalveolar carcinoma arising from a congenital cystic adenomatoid malformation in an adolescent: the first case report from the Orient. 2003;126: 902-3 (Brief comm.)

Suedkamp M (see Tossios et al). 2003;126:1513-20

Suemasu K (see Nomori et al). 2003;126:1584-9

Suematsu Y, Takamoto S. Future technologic innovations for intraoperative visualization of native coronary artery and graft anastomoses. 2003;126:304 (Letter)

Suga M (see Fischer et al). 2003;126:1174-80

Sugarbaker DJ. Discussion of Flores et al. 2003;126:11-6
Sugawara T, Sato M, Wo S, Kondo T. Possible Tx N2 M0 atypical bronchial carcinoid associated with Cushing syndrome. 2003; 126:1225 (Letter reply)

Sugi K, Kaneda Y, Sudoh M, Sakano H, Hamano K. Effect of radioisotope sentinel node mapping in patients with cT1 N0 M0 lung cancer. 2003;126:568-73

Sugi K (see Sudou et al). 2003;126:902-3 (Brief comm.)

Sullivan CC (see Chu et al). 2003;126:671-9

Sundt TM III (see Absi et al). 2003;126:344-57

Sundt TM III (see Okamoto et al). 2003;126:842-50

Sundt TM III (see Olsen et al). 2003;126:992-9

Sundt TM III (see Prasad et al). 2003;126:1822-8

Sundt TM III. Discussion of Fedak et al. 2003;126:797-806

Sutedja TW (see van der Maten et al). 2003;126:740-3

Suto T (see Takahashi et al). 2003;126:600-2 (Brief comm.)

Suzuki K (see Nakagawa et al). 2003;126:1134-40

Suzuki S (see Osawa et al). 2003;126:604-5 (Brief comm.)

Svensson LG, Kim K-H, Lytle BW, Cosgrove DM. Relationship of aortic cross-sectional area to height ratio and the risk of aortic dissection in patients with bicuspid aortic valves. 2003; 126:892-3 (Brief comm.)

Svensson LG. Discussion of Estrera et al. 2003;126:1288-95

Swain JA (see Hartz et al). 2003;126:620-2 (Editorial)

Sweet SC (see Lee et al). 2003;126:295-7 (Brief comm.)

Swistel DG (see Ashton et al). 2003;126:292-3 (Brief comm.)

Syed AU (see Border et al). 2003;126:1760-4

Szabó C (see Szabó et al). 2003;126:651-8

Szabó G, Buhmann V, Andrási T, Stumpf N, Bährle S, Kékesi V, Hagl S, Szabó C, Juhász-Nagy A. Poly-ADP-ribose polymerase inhibition protects against myocardial and endothelial reperfusion injury after hypothermic cardiac arrest. 2003;126:651-8

Szentkirályi I (see Galajda et al). 2003;126:2107 (Letter reply) Szymanowska A (see Rzyman et al). 2003;126:755-60

\section{$\mathrm{T}$}

Tabata Y (see Iwakura et al). 2003;126:1113-20

Tabayashi K (see Nitta et al). 2003;126:1186-8 (Brief comm.)

Tabayashi K (see Takahashi et al). 2003;126:1461-6

Tabley A (see Litzler et al). 2003;126:1204-7 (Brief comm.)

Taborski U (see Schoenburg et al). 2003;126:1455-60

Tada Y (see Osawa et al). 2003;126:604-5 (Brief comm.)

Tagawa T, Kozower BD, Kanaan SA, Daddi N, Suda T, Oka T, Patterson GA. Tumor necrosis factor inhibitor gene transfer ameliorates lung graft ischemia-reperfusion injury. 2003;126: 1147-54

Tagawa T (see Suda et al). 2003;126:1155-61

Tagawa T (see Yamaguchi et al). 2003;126:870-2 (Brief comm.)

Taggart DP, Djapardy V, Naik M, Davies A. A randomized trial of aprotinin (Trasylol) on blood loss, blood product requirement, and myocardial injury in total arterial grafting. 2003;126:1087-94

Taggart DP (see Browne et al). 2003;126:1061-4

Taggart DP (see Mussa et al). 2003;126:1798-805

Taguchi K (see Nakagawa et al). 2003;126:563-7

Tagusari O (see Bando et al). 2003;126:358-64

Taheri S. Myogenesis after myocardial stem cell transplantation. 2003;126:2116-7 (Letter) 
Takagi H (see Mori et al). 2003;126:415-9

Takahashi G, Sakurai M, Abe K, Itoyama Y, Tabayashi K. MCI186 prevents spinal cord damage and affects enzyme levels of nitric oxide synthase and $\mathrm{Cu} / \mathrm{Zn}$ superoxide dismutase after transient ischemia in rabbits. 2003;126:1461-6

Takahashi N (see Watanabe et al). 2003;126:1212-4 (Brief comm.)

Takahashi S, Takaya S, Fukuda I, Suto T, Daitoku K, Kuga T, Ichinoseki I, Munakata M, Fukui K, Noda H, Yodono H. Stent graft treatment for abdominal pseudoaneurysm near the celiac artery. 2003;126:600-2 (Brief comm.)

Takahashi T (see Hayashi et al). 2003;126:1813-21

Takami K (see Higashiyama et al). 2003;126:611 (Letter reply)

Takamoto S (see Higuchi et al). 2003;126:2098-100 (Brief comm.)

Takamoto S (see Imanaka et al). 2003;126:598-600 (Brief comm.)

Takamoto S (see Kitamura et al). 2003;126:904-5 (Brief comm.)

Takamoto S (see Suematsu and Takamoto). 2003;126:304 (Letter)

Takanami I. Successful treatment of huge chronic expanding hematoma after thoracoplasty. 2003;126:1202-3 (Brief comm.)

Takaya S (see Takahashi et al). 2003;126:600-2 (Brief comm.)

Takayama H (see Kitamura et al). 2003;126:904-5 (Brief comm.)

Taketatu M (see Senzaki et al). 2003;126:1645-7 (Brief comm.)

Takeuchi M (see Sano et al). 2003;126:504-10

Takeuchi Y (see Okumura et al). 2003;126:1922-8

Tamaki N (see Murashita et al). 2003;126:1328-34

Tamminen M (see Kakko et al). 2003;126:106-13

Tan Z (see Allen et al). 2003;126:1851-8

Tan Z (see Castellá et al). 2003;126:1442-8

Tanaka K (see Kanemitsu et al). 2003;126:428-35

Tanaka M, Kawahito K, Adachi H, Ino T. Platelet dysfunction in acute type A aortic dissection evaluated by the laser lightscattering method. 2003;126:837-41

Tanaka S (see Ishii et al). 2003;126:254-62

Tanel R (see Madan et al). 2003;126:1657-9 (Brief comm.)

Tarkka MR (see Wu et al). 2003;126:1477-82

Tasdemir O (see Kucuker and Tasdemir). 2003;126:2106-7 (Letter)

Tasoz R (see Sirlak et al). 2003;126:666-70

Tatoulis J (see Shah et al). 2003;126:1972-7

Tatsumi K (see Chiyo et al). 2003;126:1141-6

Tavakoli R (see Genoni et al). 2003;126:288-9 (Brief comm.)

Tayama E (see Teshima et al). 2003;126:401-7

Taylor KM (see Omar et al). 2003;126:832-6

Tedoriya T (see Date et al). 2003;126:476-81

Tei T (see Yoshimura et al). 2003;126:1417-26

Teoh K (see Gao et al). 2003;126:179-85

Tepaske R (see van den Goor et al). 2003;126:1101-6

Teshima H, Hayashida N, Yano H, Nishimi M, Tayama E, Fukunaga S, Akashi H, Kawara T, Aoyagi S. Obstruction of St Jude Medical valves in the aortic position: histology and immunohistochemistry of pannus. 2003;126:401-7

Thabut G, Mal H, Castier Y, Groussard O, Brugière O, MarrashChahla R, Lesèche G, Fournier M. Survival benefit of lung transplantation for patients with idiopathic pulmonary fibrosis. 2003; $126: 469-75$

Thankachen R (see Stephen et al). 2003;126:2093-4 (Brief comm.)

Theroux P (see Boyce et al). 2003;126:420-7

Théroux P (see Gavard et al). 2003;126:807-13

Thielmeier K (see Dowling et al). 2003;126:1271-8
Thiene G (see Bottio et al). 2003;126:99-105

Thiene G (see Rizzoli et al). 2003;126:66-74

Thistlethwaite PA, Jamieson SW. Indications for pulmonary endarterectomy. 2003;126:1228-9 (Letter reply)

Thistlethwaite PA (see Chu et al). 2003;126:671-9

Thomas R (see Naidu et al). 2003;126:200-7

Thomas R (see Naidu et al). 2003;126:482-9

Thompson RW (see Absi et al). 2003;126:344-57

Thulin L (see Boyce et al). 2003;126:420-7

Thurer RJ (see Bolooki et al). 2003;126:374-85

Tian YY (see Ad et al). 2003;126:1095-100

Tijssen JG (see van den Goor et al). 2003;126:1101-6

Tjien ATJ (see Schoof et al). 2003;126:282-3 (Brief comm.)

Toda K (see Yoshitatsu et al). 2003;126:1531-6

Tondi P (see Gaudino et al). 2003;126:1968-71

Topkara VK (see Kherani et al). 2003;126:1634-5 (Brief comm.)

Torella M (see De Santo et al). 2003;126:826-31

Tortoriello W (see Melo et al). 2003;126:914-6 (Brief comm.)

Toscano A (see Formigari et al). 2003;126:1753-9

Toscano G (see Rizzoli et al). 2003;126:66-74

Tossios P, Bloch W, Huebner A, Raji MR, Dodos F, Klass O, Suedkamp M, Kasper S-M, Hellmich M, Mehlhorn U. N-acetylcysteine prevents reactive oxygen species-mediated myocardial stress in patients, undergoing cardiac surgery: results of a randomized, double-blind, placebo-controlled clinical trial. 2003;126:1513-20

Toumpoulis IK, Anagnostopoulos CE, Drossos GE. Lack of evidence for vitamin $\mathrm{C}$ as acute vasodilator. 2003;126:1672 (Letter reply)

Trachiotis GD, Vega JD, Johnston TS, Berg A, Whelchel J, Smith AL, Lutz J, Kanter KR. Ten-year follow-up in patients with combined heart and kidney transplantation. 2003;126:2065-71

Trento A. Discussion of Greelish et al. 2003;126:365-73

Trento A. Discussion of Ikonomidis et al. 2003;126:2022-31

Triantafilou K (see Paraskevaidis et al). 2003;126:913-4 (Brief comm.)

Troconis CJ. Discussion of McMahon et al. 2003;126:1781-7

Trulock EP (see Force et al). 2003;126:864-6 (Brief comm.)

Tsang V (see McMahon et al). 2003;126:1781-7

Tscholl D (see Langer et al). 2003;126:874-5 (Brief comm.)

Tsuboi J (see Kawazoe et al). 2003;126:908-10 (Brief comm.)

Tsuboshima K (see Okada et al). 2003;126:1911-5

Tsubota N (see Okada et al). 2003;126:1911-5

Tsuchiya K (see Naito et al). 2003;126:883-5 (Brief comm.)

Tsuchiya R (see Nakagawa et al). 2003;126:1134-40

Tsui S (see Lim et al). 2003;126:1013-7

Tsui S (see Stoica et al). 2003;126:2072-4 (Brief comm.)

Tsuneyoshi M (see Yamaguchi et al). 2003;126:870-2 (Brief comm.)

Tsuru Y (see Nitta et al). 2003;126:1186-8 (Brief comm.)

Tucker OP (see Duncan et al). 2003;126:694-702

Tufail A (see Feindel et al). 2003;126:777-82

Tulzer G (see Mair et al). 2003;126:1378-84

Tung WS (see Absi et al). 2003;126:344-57

Turina J (see Kaemmerer et al). 2003;126:1048-52

Turina M (see Genoni et al). 2003;126:288-9 (Brief comm.)

Tweddell JS (see Ghanayem et al). 2003;126:1367-77

Tzialtas D (see Colli et al). 2003;126:881-3 (Brief comm.) 
U

Uchino K (see Okada et al). 2003;126:558-62

Uchino K (see Okada et al). 2003;126:1911-5

Uchiyama T (see Uchiyama et al). 2003;126:148-59

Uchiyama Y, Otani H, Okada T, Uchiyama T, Ninomiya H, Kido M, Imamura H, Nakao S, Shingu K. Integrated pharmacological preconditioning in combination with adenosine, a mitochondrial $\mathrm{K}_{\mathrm{ATP}}$ channel opener and a nitric oxide donor. 2003; 126:148-59

Ueda T (see Nakagawa et al). 2003;126:563-7

Ueno T (see Hirata et al). 2003;126:1080-6

Uhlig A (see Gulbins et al). 2003;126:890-1 (Brief comm.)

Umeda Y (see Mori et al). 2003;126:415-9

Ungerleider R (see Dickey et al). 2003;126:1259-60 (Editorial)

Unimonh O (see Koyama et al). 2003;126:2110-1 (Letter reply)

Urbanski PP. Cannulation of the left common carotid artery for proximal aortic repair. 2003;126:887-8 (Brief comm.)

Urschel HC Jr. Robert W. Jamplis (1920-2003). 2003;126:311-2 (In Memoriam)

Uusimaa P (see Kakko et al). 2003;126:106-13

Uzark KC (see Border et al). 2003;126:1760-4

\section{V}

Va Rooijen N (see Naidu et al). 2003;126:200-7

Vacanti JP (see Grikscheit et al). 2003;126:537-44

Vaccarino V, Koch CG. Long-term benefits of coronary bypass surgery: are the gains for women less than for men? 2003;126: 1707-11 (Editorial)

Vaccarino V (see Koch et al). 2003;126:932-5 (Editorial)

Valente M (see Bottio et al). 2003;126:99-105

Valfré C (see Bottio et al). 2003;126:99-105

Vallieres E. Discussion of Flores et al. 2003;126:11-6 van Andel CJ (see Scheltes et al). 2003;126:191-9 (Evolving tech.) van Boven WJ (see Borst et al). 2003;126:305 (Letter reply) van den Brink A (see van den Goor et al). 2003;126:1101-6 van den Goor JM, van den Brink A, Nieuwland R, van Oeveren W,

Rutten PM, Tepaske R, Tijssen JG, Sturk A, de Mol BA, Eijsman L. Generation of platelet-derived microparticles in patients undergoing cardiac surgery is not affected by complement activation. 2003;126:1101-6

van der Maten J, Blaauwgeers JLG, Sutedja TG, Kwa HB, Postmus PE, Wagenaar SjSc. Granular cell tumors of the tracheobronchial tree. 2003;126:740-3

van Dongen EI, Glansdorp AG, Mildner RJ, McCrindle BW, Sakopoulos AG, VanArsdell G, Williams WG, Bohn D. The influence of perioperative factors on outcomes in children aged less than 18 months after repair of tetralogy of Fallot. 2003; 126:703-10

van Doorn C (see McMahon et al). 2003;126:1781-7

van Oeveren W (see van den Goor et al). 2003;126:1101-6

Van Raemdonck D (see Lerut et al). 2003;126:1121-8

van Vliet S (see Grauss et al). 2003;126:2003-10

VanArsdell G (see van Dongen et al). 2003;126:703-10

Vanermen H. Discussion of Schmitz et al. 2003;126:1829-38
Vannan MA, Sarkar K. Assessment of mechanical aortic valve prosthesis by means of Doppler echocardiography: what to measure and why? 2003;126:317-20 (Editorial)

Vaporciyan AA (see Maish and Vaporciyan). 2003;126:2077-80 (Brief comm.)

Varela A (see Hernández et al). 2003;126:2081-2 (Brief comm.)

Vargas FJ. Discussion of McMahon et al. 2003;126:1781-7

Vega JD (see Trachiotis et al). 2003;126:2065-71

Velez DA (see Corvera et al). 2003;126:1549-54

Verbalis J (see Ad et al). 2003;126:1095-100

Verhoeven ELG (see Drenth et al). 2003;126:1184-5 (Brief comm.)

Verma S, Badiwala MV, Weisel RD, Li S-H, Wang C-H, Fedak PWM, Li R-K, Mickle DAG. C-reactive protein activates the nuclear factor- $\kappa \mathrm{B}$ signal transduction pathway in saphenous vein endothelial cells: implications for atherosclerosis and restenosis. 2003;126:1886-91

Verma S, Fedak PWM, Ko L, Cusimano RJ, Walton NA, Parker JD, Yau TM. Evaluation of a novel sutureless anastomotic connector: from endothelial function to mid-term clinical and angiographic follow-up. 2003;126:1555-60 (Evolving tech.)

Verma S (see Fedak et al). 2003;126:797-806

Veronesi G, Leon ME, Spaggiari L. Safety of bronchoplastic resection after induction therapy for lung cancer. 2003;126: 1670-1 (Letter)

Veronesi G (see Pastorino et al). 2003;126:1906-10

Verrier E. Discussion of Greelish et al. 2003;126:365-73

Verrier E. Discussion of Lupinetti et al. 2003;126:240-6

Verrier ED (see Naidu et al). 2003;126:200-7

Versteegh MIM (see Braun et al). 2003;126:284-6 (Brief comm.)

Vetter V (see Madan et al). 2003;126:1657-9 (Brief comm.)

Vicol C (see Nollert et al). 2003;126:1192-4 (Brief comm.)

Vigilance DW (see Kherani et al). 2003;126:1634-5 (Brief comm.)

Vigilance DW (see Morgan et al). 2003;126:582-3 (Brief comm.)

Vigilance DW (see Morgan et al). 2003;126:1188-90 (Brief comm.)

Vigilance DW (see Morgan et al). 2003;126:1624-33

Vincent R (see Dasika et al). 2003;126:2112 (Letter)

Vinnerkvist A (see Estrera et al). 2003;126:1288-95

Vinten-Johansen J, Mentzer RM Jr. Attenuation of postcardioplegia injury with inhibitors of the sodium-hydrogen exchanger. 2003;126:1265-7 (Editorial)

Vinten-Johansen J (see Corvera et al). 2003;126:1549-54

Vinten-Johansen J. Discussion of Verma et al. 2003;126:1886-91

Visconti KJ (see Jonas et al). 2003;126:1765-74

Vitolla G (see Gaudino et al). 2003;126:1076-9

Vivda V (see Bottio et al). 2003;126:1352-6

Vogt DP (see Lam et al). 2003;126:2101-3 (Brief comm.)

Vogt PR (see Schoenburg et al). 2003;126:1455-60

Voigt PG (see Braun et al). 2003;126:284-6 (Brief comm.)

Voisine P (see Ruel et al). 2003;126:1521-30

Vollandt R (see Albes et al). 2003;126:1504-12

von Heymann C, Ziemer S, Kox WJ, Spies C. Caveat against the use of FEIBA in combination with recombinant factor VIIa. 2003;126:1667-8 (Letter)

von Segesser LK (see Corno et al). 2003;126:1775-80

von Segesser LK. Discussion of Estrera et al. 2003;126:1288-95

von Segesser LK. Discussion of Sergeant et al. 2003;126:1279-87 
Vorp D (see Bonanomi et al). 2003;126:851-2 (Evolving tech.)

Vouhé P (see Boudjemline et al). 2003;126:490-7

Vouhé P (see Corno et al). 2003;126:1775-80

\section{W}

Wade DT (see Browne et al). 2003;126:1061-4

Wagenaar SjSc (see van der Maten et al). 2003;126:740-3

Wahba A. Discussion of Verma et al. 2003;126:1886-91

Wahlers T (see Albes et al). 2003;126:1504-12

Waight D (see Bacha et al). 2003;126:1718-23

Wain JC (see Gaissert et al). 2003;126:744-7

Waldmann M, Chen RP-C, Armour JA. Surgery modifies cardiac sensory transduction. 2003;126:1792-7

Walhout RJ, Lekkerkerker JC, Oron GH, Hitchcock FJ, Meijboom EJ, Bennink GBWE. Comparison of polytetrafluoroethylene patch aortoplasty and end-to-end anastomosis for coarctation of the aorta. 2003;126:521-8

Wallard M (see Lim et al). 2003;126:1013-7

Walles T (see Leyh et al). 2003;126:1000-4

Walterbusch G (see Cebi et al). 2003;126:2074-7 (Brief comm.)

Walters HL III (see Delius et al). 2003;126:2095-6 (Brief comm.)

Walther C (see Falk et al). 2003;126:1575-9 (Evolving tech.)

Walther T (see Falk et al). 2003;126:1575-9 (Evolving tech.)

Walton NA (see Verma et al). 2003;126:1555-60 (Evolving tech.)

Wandschneider W, Illiasch H. Intralobar sequestration: a rare cause of severe hemothorax. 2003;126:872-3 (Brief comm.)

Wang C-H (see Verma et al). 2003;126:1886-91

Wang F-Y (see Yu et al). 2003;126:80-9

Wang $\mathrm{J}$ (see $\mathrm{He}$ et al). 2003;126:56-65

Wang M-J (see Lin et al). 2003;126:2011-5

Wang N (see Wolf et al). 2003;126:168-78

Ward D (see Jahangiri et al). 2003;126:894-895 (Brief comm.) Correction 2003;126:1680

Warren AE (see Baskett et al). 2003;126:232-9

Warren M (see Williams et al). 2003;126:436-41

Watanabe A, Watanabe T, Obama T, Ohsawa H, Mawatari T, Ichimiya Y, Takahashi N, Abe T. New material for reconstruction of the anterior chest wall, including the sternum. 2003; 126:1212-4 (Brief comm.)

Watanabe H (see Nakagawa et al). 2003;126:563-7

Watanabe T (see Watanabe et al). 2003;126:1212-4 (Brief comm.)

Waterbolk TW (see Drenth et al). 2003;126:1184-5 (Brief comm.)

Wauthy P, Kafi SA, Mooi WJ, Naeije R, Brimioulle S. Inhaled nitric oxide versus prostacyclin in chronic shunt-induced pulmonary hypertension. 2003;126:1434-41

Wechsler AS. The editorial board of The Journal of Thoracic and Cardiovascular Surgery. 2003;126:1-4 (Editorial)

Wechsler AS, Fried PW. Peer review. 2003;126:1681-2 (Editorial)

Wechsler AS. The gender initiative. 2003;126:617 (Editorial)

Wechsler AS. Priority issue: who is on first base? 2003;126:322 (Editorial response)

Wechsler AS (see Rangappa et al). 2003;126:124-32

Wechsler AS (see Samuels et al). 2003;126:2100-1 (Brief comm.)

Wechsler AS. Discussion of Baskett et al. 2003;126:232-9

Weinberg A (see Selzman et al). 2003;126:688-93

Weinberg AD (see Morgan et al). 2003;126:1624-33

Weiner LM (see Goldberg et al). 2003;126:1168-73
Weinreich S (see Schmitz et al). 2003;126:1829-38

Weinstein S, Chan D, Davis JT. Outcome of symptomatic patients undergoing extracardiac Fontan conversion and cryoablation. 2003;126:529-36

Weisel RD (see Fazel et al). 2003;126:2114-6 (Letter reply)

Weisel RD (see Fedak et al). 2003;126:797-806

Weisel RD (see Ohno et al). 2003;126:1537-48

Weisel RD (see Verma et al). 2003;126:1886-91

Weitzman MD (see Chu et al). 2003;126:671-9

Wells W, Malas M, Baker CJ, Quardt SM, Barr ML. Depopulated vena caval homograft: a new venous conduit. 2003;126:498503

Welz A (see Schmitz et al). 2003;126:645-50

Welz A (see Schmitz et al). 2003;126:1829-38

Wendler O (see Langer et al). 2003;126:874-5 (Brief comm.)

Wenger NK. Is what's good for the gander good for the goose? 2003;126:929-31 (Editorial)

Wernly JA (see Dietl et al). 2003;126:1181-3 (Brief comm.)

Wernovsky G (see Bellinger et al). 2003;126:1385-96

Wernovsky G (see Gaynor et al). 2003;126:1736-45

Wernovsky G (see Wypij et al). 2003;126:1397-403

Westaby S. Discussion of Pilla et al. 2003;126:1467-76

Whelchel J (see Trachiotis et al). 2003;126:2065-71

White J (see Schmitz et al). 2003;126:1829-38

White JA (see Banbury et al). 2003;126:680-7

White PC (see Yarbrough et al). 2003;126:1892-9

White RD (see Setser et al). 2003;126:48-55

White W (see Scarborough et al). 2003;126:1561-7 (Evolving tech.)

Whitmore P (see McMahon et al). 2003;126:1781-7

Whitten CW (see Greilich et al). 2003;126:1498-503

Whooley BP (see Murthy et al). 2003;126:1162-7

Whyte R. Discussion of Roberts et al. 2003;126:1597-602

Wilhelm W (see Langer et al). 2003;126:874-5 (Brief comm.)

Wilhelmi M (see Leyh et al). 2003;126:1000-4

Wilimski R (see Rzyman et al). 2003;126:755-60

Williams M, Casher J, Joshi N, Hankinson T, Warren M, Oz M, Naka Y, Mancini D. Insertion of a left ventricular assist device in patients without thorough transplant evaluations: a worthwhile risk? 2003;126:436-41

Williams RV (see Hawkins et al). 2003;126:247-53

Williams WG (see van Dongen et al). 2003;126:703-10

Wilson DA (see Kherani et al). 2003;126:1634-5 (Brief comm.)

Wilson MK (see Beldholm et al). 2003;126:545-50

Wimmer-Greinecker G, Dogan S, Aybek T, Khan MF, Mierdl S, Byhahn C, Moritz A. Totally endoscopic atrial septal repair in adults with computer-enhanced telemanipulation. 2003;126: 465-8 (Evolving tech.)

Wimmer-Greinecker G (see Falk et al). 2003;126:1575-9 (Evolving tech.)

Wimmer-Greinecker G (see Klima et al). 2003;126:1568-74 (Evolving tech.)

Wittnich C (see Bandali et al). 2003;126:1730-5

Wo S (see Sugawara et al). 2003;126:1225 (Letter reply)

Woehleke T (see Doss et al). 2003;126:814-7

Wolf PL (see Chu et al). 2003;126:671-9

Wolf RK, Alderman EL, Caskey MP, Raczkowski AR, Dullum MK, Lundell DC, Hill AC, Wang N, Daniel MA. Clinical and 
six-month angiographic evaluation of coronary arterial graft interrupted anastomoses by use of a self-closing clip device: a multicenter prospective clinical trial. 2003;126:168-78

Wolf RK, Falk V. Intraoperative assessment of coronary artery bypass grafts. 2003;126:634-7 (Editorial)

Wolfe W. Discussion of Lai et al. 2003;126:1978-86

Wolfe WG (see Domkowski et al). 2003;126:386-90

Wolner E (see Czerny et al). 2003;126:916-8 (Brief comm.)

Wolner E (see Czerny et al). 2003;126:1296-301

Wong J (see Murthy et al). 2003;126:1162-7

Woo YJ. Discussion of Verma et al. 2003;126:1886-91

Woo YJ. Discussion of Yarbrough et al. 2003;126:1892-9

Wood DE. Discussion of DeCamp et al. 2003;126:17-27

Wood DE. Discussion of Gonzalez-Stawinski et al. 2003;126: 1900-5

Wood DE. Discussion of Roberts et al. 2003;126:1597-602

Wood JP (see Doss et al). 2003;126:814-7

Woolley SM (see Naidu et al). 2003;126:200-7

Woolley SM (see Naidu et al). 2003;126:482-9

Woolson SL (see Reed et al). 2003;126:1943-51

Wooten MK (see Domkowski et al). 2003;126:386-90

Wouters P (see Sergeant et al). 2003;126:1279-87

Wright CD (see Gaissert et al). 2003;126:744-7

Wu EX (see He et al). 2003;126:56-65

$\mathrm{Wu}$ YX, Grunkemeier G. Problems with complication rate analysis. 2003;126:1670 (Letter reply)

Wu YX (see Grunkemeier and Wu). 2003;126:1661 (Letter reply)

Wu Z-K, Iivainen T, Pehkonen E, Laurikka J, Zhang S, Tarkka MR. Fibrillation in patients subjected to coronary artery bypass grafting. 2003;126:1477-82

Wypij D, Newburger JW, Rappaport LA, duPlessis AJ, Jonas RA, Wernovsky G, Lin M, Bellinger DC. The effect of duration of deep hypothermic circulatory arrest in infant heart surgery on late neurodevelopment: the Boston Circulatory Arrest Trial. 2003; 126:1397-403

Wypij D (see Bellinger et al). 2003;126:1385-96

Wypij D (see Jonas et al). 2003;126:1765-74

\section{$x$}

Xia F (see Xia et al). 2003;126:1404-10

Xia Z, Gu J, Ansley DM, Xia F, Yu J. Antioxidant therapy with Salvia miltiorrhiza decreases plasma endothelin-1 and thromboxane B2 after cardiopulmonary bypass in patients with congenital heart disease. 2003;126:1404-10

Xia Z (see Ansley et al). 2003;126:1222-3 (Brief comm.)

Xu H (see Okamoto et al). 2003;126:842-50

Xu S (see Ruel et al). 2003;126:1521-30

\section{Y}

Yabuki S (see Aramaki et al). 2003;126:853-4 (Brief comm.)

Yacoub M (see Blackstone et al). 2003;126:783-96

Yacoub MH. Discussion of Fedak et al. 2003;126:797-806

Yada I (see Kanemitsu et al). 2003;126:428-35

Yagihara T (see Bando et al). 2003;126:358-64

Yamad RE (see Mahtabifard et al). 2003;126:28-38
Yamada A, Morishita K, Kawaharada N, Fukada J, Satsu T, Abe T. A safe strategy for surgical repair of coarctation of the aorta in an adult. 2003;126:597-8 (Brief comm.)

Yamada RE (see Merritt et al). 2003;126:1609-17

Yamagishi M, Shuntoh K, Matsushita T, Fujiwara K, Shinkawa T, Miyazaki T, Kitamura N. Mitral valve replacement by a GoreTex reinforced pulmonary autograft in a child. 2003;126:1218-9 (Brief comm.)

Yamaguchi M, Yoshino I, Osoegawa A, Kameyama T, Tagawa T, Fukuyama S, Oda Y, Tsuneyoshi M, Maehara Y. Inflammatory myofibroblastic tumor of the mediastinum presenting as superior vena cava syndrome. 2003;126:870-2 (Brief comm.)

Yamaguchi M (see Ootaki et al). 2003;126:1411-6

Yamaguchi M (see Ootaki et al). 2003;126:1655-6 (Brief comm.)

Yamaguchi M (see Yoshimura et al). 2003;126:1417-26

Yamamoto Y (see Gotoh et al). 2003;126:1916-21

Yamaya K (see Nitta et al). 2003;126:1186-8 (Brief comm.)

Yang H (see Gao et al). 2003;126:179-85

Yang Q (see Zou et al). 2003;126:208-15

Yankah C. Discussion of Sergeant et al. 2003;126:1279-87

Yano H (see Teshima et al). 2003;126:401-7

Yarbrough WM, Mukherjee R, Escobar GP, Mingoia JT, Sample JA, Hendrick JW, Dowdy KB, McLean JE, Stroud RE, Spinale FG. Direct inhibition of the sodium/hydrogen exchanger after prolonged regional ischemia improves contractility on reperfusion independent of myocardial viability. 2003;126:1489-97

Yarbrough WM, Mukherjee R, Escobar GP, Sample JA, McLean JE, Dowdy KB, Hendrick JW, Gibson WC, Hardin AE, Mingoia JT, White PC, Stiko A, Armstrong RC, Crawford FA, Spinale FG. Pharmacologic inhibition of intracellular caspases after myocardial infarction attenuates left ventricular remodeling: a potentially novel pathway. 2003;126:1892-9

Yasuda K (see Murashita et al). 2003;126:1328-34

Yasufuku K (see Chiyo et al). 2003;126:1141-6

Yasui H (see He et al). 2003;126:455-64

Yatsu S (see Nishimura et al). 2003;126:1190-2 (Brief comm.)

Yau TM (see Davierwala et al). 2003;126:1335-44

Yau TM (see Verma et al). 2003;126:1555-60 (Evolving tech.)

Yaun Q (see Pilla et al). 2003;126:1467-76

Yavuzer Ş (see Şahin et al). 2003;126:769-73

Yazaki A (see Kanemitsu et al). 2003;126:428-35

Yazicioglu L (see Sirlak et al). 2003;126:666-70

Yeh T Jr, Austin EH III, Sehic A, Edmonds HL Jr. Rapid recognition and treatment of cerebral air embolism: the role of neuromonitoring. 2003;126:589-91 (Brief comm.)

Yi G-h (see He et al). 2003;126:56-65

Yilmaz O (see Sonmez et al). 2003;126:1662-3 (Letter)

Yim APC (see Zou et al). 2003;126:208-15

Ylitalo A (see Kakko et al). 2003;126:106-13

Yodh AG (see Friedberg et al). 2003;126:1963-7 (Evolving tech.) Yodono H (see Takahashi et al). 2003;126:600-2 (Brief comm.)

Yoganathan A (see Saxena et al). 2003;126:90-8

Yokomise H (see Gotoh et al). 2003;126:1916-21

Yokote Y (see Senzaki et al). 2003;126:1645-7 (Brief comm.)

Yokouchi H (see Higashiyama et al). 2003;126:611 (Letter reply) Yoshiba S (see Fukuda et al). 2003;126:1220-1 (Brief comm.)

Yoshida M (see Ootaki et al). 2003;126:1411-6

Yoshida M (see Ootaki et al). 2003;126:1655-6 (Brief comm.) 
Yoshida M (see Sakiyama et al). 2003;126:1207-9 (Brief comm.)

Yoshida M (see Yoshimura et al). 2003;126:1417-26

Yoshida S (see Chiyo et al). 2003;126:1141-6

Yoshida S (see Sakiyama et al). 2003;126:1207-9 (Brief comm.)

Yoshii S (see Osawa et al). 2003;126:604-5 (Brief comm.)

Yoshikawa M (see Yoshitatsu et al). 2003;126:1531-6

Yoshimura N,Yamaguchi M, Ohashi H, Oshima Y, Oka S, Yoshida M, Murakami H, Tei T. Pulmonary atresia with intact ventricular septum: strategy based on right ventricular morphology. 2003;126:1417-26

Yoshimura N (see Ootaki et al). 2003;126:1411-6

Yoshimura N (see Ootaki et al). 2003;126:1655-6 (Brief comm.)

Yoshino I (see Yamaguchi et al). 2003;126:870-2 (Brief comm.)

Yoshitatsu M, Miyamoto Y, Mitsuno M, Toda K, Yoshikawa M, Fukui S, Nomura F, Hirata N, Onishi K. Changes in left anterior descending coronary artery flow profiles after coronary artery bypass grafting examined by means of transthoracic Doppler echocardiography. 2003;126:1531-6

Yu H-Y, Ho Y-L, Chu S-H, Chen Y-S, Wang S-S, Lin F-Y. Long-term evaluation of Carpentier-Edwards porcine bioprosthesis for rheumatic heart disease. 2003;126:80-9

Yu J (see Xia et al). 2003;126:1404-10

Yun J (see Fischbein et al). 2003;126:216-23

\section{Z}

Zacharias J, Clark SC, Hamilton JRL, Dark JH, Hasan A. Unilateral pulmonary thromboendarterectomy for iatrogenic pulmo- nary hypertension in a ten-year-old child. 2003;126:1210-1 (Brief comm.)

Zackai EH (see Gaynor et al). 2003;126:1736-45

Zamparelli R (see Gaudino et al). 2003;126:1107-12

Zamvar V, Sivaprakasam R. The left anterior descending coronary artery is the best recipient. 2003;126:923 (Letter)

Zegdi R, Fabre O, Lila N, Fornès P, Cambillau M, Shen M, Hervé P, Carpentier A, Fabiani J-N. Exhaled carbon monoxide and inducible heme oxygenase expression in a rat model of postperfusion acute lung injury. 2003;126:1867-74

Zenati MA (see Bonanomi et al). 2003;126:851-2 (Evolving tech.)

Zenati MA (see Bonanomi et al). 2003;126:1859-66

Zerkowski H-R (see Georgiadis et al). 2003;126:1638-9 (Brief comm.)

Zhang S (see Wu et al). 2003;126:1477-82

Zielinsky P (see Assad et al). 2003;126:300-2 (Brief comm.)

Ziemer S (see von Heymann et al). 2003;126:1667-8 (Letter)

Zimmerman MA (see Selzman et al). 2003;126:688-93

Zimpfer D (see Czerny et al). 2003;126:916-8 (Brief comm.)

Zimpfer D (see Czerny et al). 2003;126:1296-301

Zitser-Gurevich Y (see Galai et al). 2003;126:1018-25

Zoffoli G (see Colli et al). 2003;126:881-3 (Brief comm.)

Zou W, Yang Q, Yim APC, He G-W. Impaired endotheliumderived hyperpolarizing factor-mediated relaxation in porcine pulmonary microarteries after cold storage with Euro-Collins and University of Wisconsin solutions. 2003;126:208-15

Zrenner B (see Kaemmerer et al). 2003;126:1048-52

\section{Subject Index*}

A

Abnormalities; see also specific abnormality

Anomalous origin of the left coronary artery from the main pulmonary artery associated with Berry syndrome (Senzaki et al). 2003;126:1645-7 (Brief comm.)

Benign intrapulmonary teratoma: report of a case (Eren et al). 2003;126:855-7 (Brief comm.)

Bilateral lung transplantation for pulmonary hypoplasia caused by congenital diaphragmatic hernia (Lee et al). 2003;126:295-7 (Brief comm.)

Congenital thoracoabdominal aortic aneurysm (Ko et al). 2003;126:897-9 (Brief comm.)

Emergency hospital admissions and three-year survival of adults with and without cardiovascular surgery for con-

*July, 1-310; August 311-616; September, 617-928; October, 929-1234; November, 1235-1680; December, 1681-2202. genital cardiac disease (Kaemmerer et al). 2003;126:1048-52

New lead for in utero pacing for fetal congenital heart block (Assad et al). 2003;126:300-2 (Brief comm.)

Pulmonary blood pressure, not flow, is associated with net endothelin-1 production in the lungs of patients with congenital heart disease and normal pulmonary vascular resistance (Fratz et al). 2003;126:1724-9

Rapid progression of midventricular obstruction in adults with double-chambered right ventricle (Oliver et al). 2003; 126:711-7

Relationship of aortic cross-sectional area to height ratio and the risk of aortic dissection in patients with bicuspid aortic valves (Svensson et al). 2003;126:892-3 (Brief comm.)

Successful Fontan procedure for asplenia with pulmonary atresia and major aortopulmonary collateral arteries (Miyaji et al). 2003;126:1648-50 (Brief comm.) 